\title{
Review
}

\section{TWO-DIMENSIONAL OPTICAL SPECTROSCOPY OF MOLECULAR AGGREGATES}

\author{
V. Butkus 国, D. Abramavicius 回, A. Gelzinis 目, and L. Valkunas 明 \\ ${ }^{a}$ Department of Theoretical Physics, Faculty of Physics of Vilnius University, Sauletekio 9, LT-10222 Vilnius, Lithuania \\ E-mail: vytautas.butkus@ff.vu.lt \\ ${ }^{\mathrm{b}}$ State Key Laboratory of Supramolecular Complexes, Jilin University, 2699 Qianjin Street, Changchun 130012, PR China \\ ${ }^{\mathrm{c}}$ Center for Physical Sciences and Technology, Savanoriu 231, LT-02300 Vilnius, Lithuania
}

Received 10 September 2010; accepted 16 September 2010

\begin{abstract}
An overview of the theoretical background for the novel spectroscopic tool - two-dimensional (2D) optical spectroscopy is presented. Principles of nonlinear polarization induction, signal generation, and detection are described. Concepts of heterodyned four-wave mixing experimental technique and 2D spectra construction are detailed and the scheme of third-order polarization calculation is consistently introduced. The system response function theory is formulated for a general multi-level quantum system considering the system-field interaction perturbatively. Equations of motion for the system density operator relevant to the third-order response are presented. Basic quantum systems of a two-level atom, two-level molecule, and a three-level system are considered and analytic expressions of the third-order signal are derived at certain limits. Molecular complexes are described using the Frenkel exciton approach. 2D spectra of the excitonically-coupled dimers of two-level and three-level chromophores are presented. Possibilities of extraction of separate spectral elements as well as performing quantum control by the two-colour 2D spectroscopy for the dimer of excitonically-coupled two-level systems are demonstrated. Effects of motional narrowing of one-dimensional J-aggregates of pseudoisocyanine and construction of the J-band as well as highlyefficient excitonic energy transfer in photosynthetic Fenna-Matthews-Olson complex are illustrated by simulated time-resolved 2D spectra.
\end{abstract}

Keywords: optical two-dimensional spectroscopy, four-wave mixing, molecular excitons

PACS: 78.47.nj, 78.47.Fg, 71.35.Aa 


\section{Contents}

\begin{tabular}{llr}
\hline 1 & Introduction & 269
\end{tabular}

2 Theoretical background $\quad 270$

2.1 Semi-classical concept of the excitation and measurement . . . . . . . . . . . . . . . . . . . 270

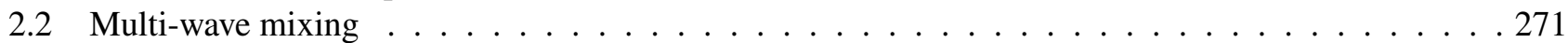

2.3 Signal detection . . . . . . . . . . . . . . . . . . . . . . . . . . . . 271

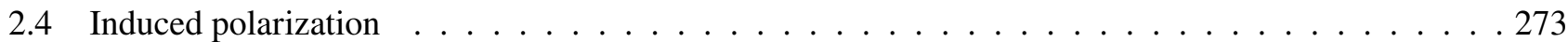

2.4 .1 The Liouville-space and reduced density matrix . . . . . . . . . . . . . . . . . 273

2.4 .2 Series expansion of the density matrix . . . . . . . . . . . . . . . . . . . 274

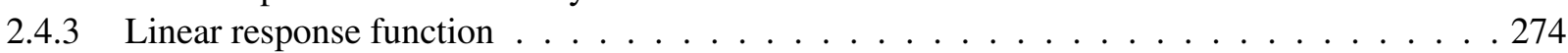

2.4 .4 The third-order response function . . . . . . . . . . . . . . . . . . . 275

2.4 .5 A non-perturbative propagation of the density matrix . . . . . . . . . . . . . . 276

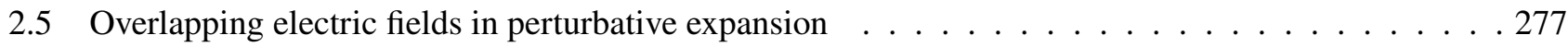

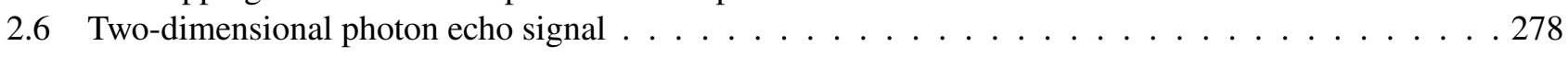

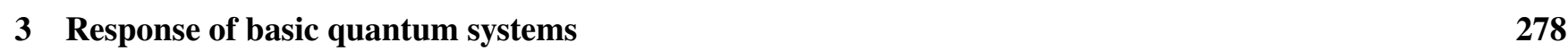

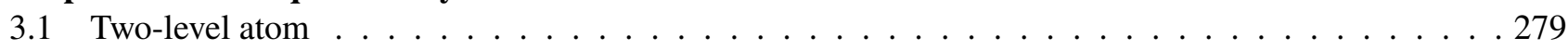

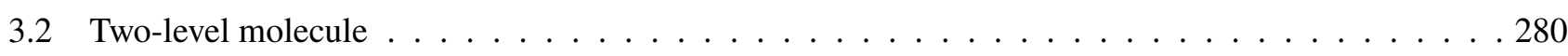

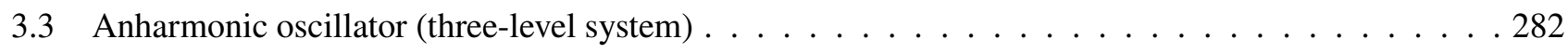

3.4 Multi-level system in contact with the bath: cumulant expansion of the third-order response function 283

4 Coupled multi-chromophore system 285

4.1 Frenkel exciton model . . . . . . . . . . . . . . . . . . . . . . . . . 285

4.2 System response function of three-manifold system $\ldots \ldots \ldots \ldots \ldots$. . . . . . . . . 287

5 Spectroscopy of dimers $\quad 287$

5.1 Dimer of two-level chromophores ．. . . . . . . . . . . . . . . . . . . . . . . . 287

$5.2 \quad$ Excitonically coupled dimer: signatures of different evolution scenarios and pulse-overlap effects in

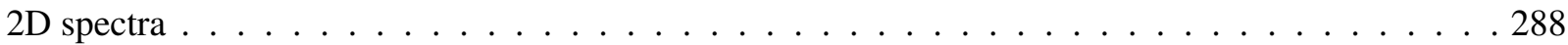

$5.2 .1 \quad$ Broad-bandwidth pulse simulations . . . . . . . . . . . . . . . . . . . . 288

5.2.2 Gaussian narrow-bandwidth simulations . . . . . . . . . . . . . . . . . . . . . . . . . . . . 290

5.3 Dimer of three-level chromophores . . . . . . . . . . . . . . . . . . . . . . . . . 292

6 Applications to multi-chromophore complexes 294

6.1 One-dimensional J-aggregates of pseudoisocyanine . . . . . . . . . . . . . . . . . . . . . . . . . 294

6.2 Excitation transfer pathways in Fenna-Matthews-Olson photosynthetic complex . . . . . . . . . . 296

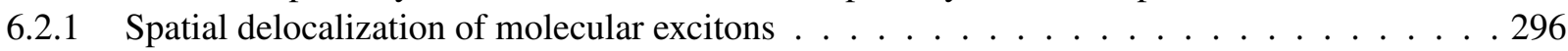

6.2 .2 Energy transfer pathways . . . . . . . . . . . . . . . . . . . . . . 297

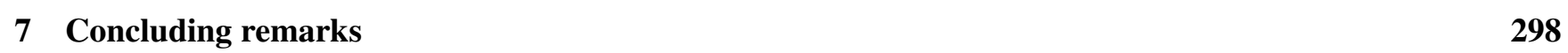

\begin{tabular}{|l|l}
\hline Appendices & 299
\end{tabular}

\begin{tabular}{|l|l|l|l|l}
\hline A. The cumulant expansion & 299
\end{tabular}

B. Response function of an open excitonic system with diagonal and off-diagonal fluctuations 299

\begin{tabular}{ll}
\hline References & 301
\end{tabular} 


\section{Introduction}

Nonlinear optical techniques performed using ultrashort laser pulses in regions from IR to visible wavelengths are capable of probing various dynamical phenomena on microscopic/nanoscopic scale. The fourwave mixing (FWM) experiments are the simplest nonlinear techniques available for isotropic systems [1, 2]. In the time domain, experiments are performed by applying either two-pulses (pump-probe) or three-pulses (homodyne three-pulse photon echo), or four-pulses (coherent heterodyned signals) to generate and detect the desired signal. The FWM signal is generated by the induced third-order polarization, which is a parametric function of the delays between the adjacent laser pulses. The polarization dynamics with respect to these parameters reflect wide variety of ultrafast molecular processes.

Recent development of nonlinear spectroscopies, such as two-dimensional photon echo (2D PE) spectroscopy, is getting widely available for studies of exciton coherence in various molecular systems [3-5]. Molecular dimer is the simplest system, where the spectral features illustrating dynamics of quantum coherence are expected [6-9]. Main features of one-colour and multi-colour 2D PE spectra of a dimer are well described theoretically [7, 8, 10-12]. 2D PE spectroscopy was the key tool demonstrating a complex pathway network of the energy transfer and long-lasting coherence in a photosynthetic Fenna-Matthews-Olson (FMO) complex [13-15], as well as in LH3 complexes from photosynthetic bacteria [4]. Recently the 2D PE spectra have also been recorded for conjugated polymers [16] and cylindrical (bi-tubular) J-aggregates [17, 18]. Apart from clear identification of exciton transfer between the tubes, quantum coherence oscillations were also observed. By analysing the J-band of one-dimensional $\mathrm{J}$-aggregate it was demonstrated that 2D PE spectroscopy visualizes the intra-band heterogenous dephasing dynamics [19] and intra-band coherences [17].

Sophisticated experimental techniques have been developed in the visible optical region maintaining coherence between pulses [20, 21]. In 2D optical spectroscopy, an experimental FWM scheme with heterodyne detection is applied (Fig. 1). Three wave vectors of ultra-short laser pulses exciting the sample at times $\tau_{1}, \tau_{2}$, and $\tau_{3}$ are denoted as $\mathbf{k}_{1}, \mathbf{k}_{2}$, and $\mathbf{k}_{3}$, respectively. The induced third-order polarization within the sample is the source of the radiated electric field. Due to nonlinear processes undergoing within the sample the signal

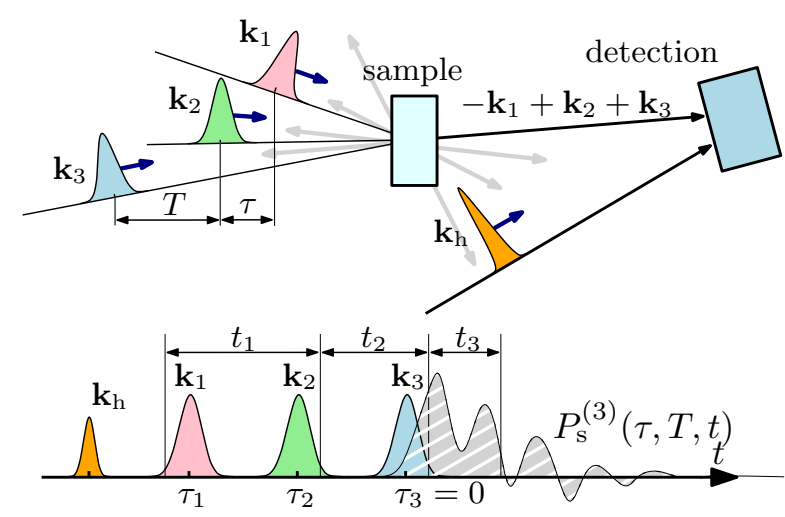

Fig. 1. 2D PE experimental scheme with heterodyne detection and definitions of variables in the lower panel: $\tau_{1}, \tau_{2}$, and $\tau_{3}$ are the times of three laser pulses exciting the sample, while $t_{1}, t_{2}$, and $t_{3}$ are the time variables for the system response function.

is generated along directions $\pm \mathbf{k}_{1} \pm \mathbf{k}_{2} \pm \mathbf{k}_{3}$. The signal exclusively generated in $\mathbf{k}_{\mathrm{s}}=-\mathbf{k}_{1}+\mathbf{k}_{2}+\mathbf{k}_{3}$ direction is denoted as the photon echo signal. Since the absolute interaction time is irrelevant due to the system being in the thermal equilibrium before the first interaction, the PE signal can be characterized as a three-variable function $W(\tau, T, t)$ of positive delay times between successive laser pulses $\tau \equiv \tau_{2}-\tau_{1}$ and $T \equiv \tau_{3}-\tau_{2}$ and the detection time $t$. The time delays between adjacent laser pulses ( $\tau$ and $T$ ) are controlled with a high precision. A two-dimensional one-sided Fourier transform of the first delay time interval and the signal detection time, that is, $\tau \rightarrow \omega_{\tau}$ and $t \rightarrow \omega_{t}$, is applied [13, 20, 22-24]. The transformed data can be plotted as two-dimensional spectrograms with respect to the second delay time $T$ (Fig. 2). Diagonal peaks, $\omega_{\tau}=\left|\omega_{t}\right|$, reflect the exciton eigenstates and off-diagonal peaks, $\omega_{t} \neq\left|\omega_{\tau}\right|$, show correlations and coherences within the system due to many-exciton interactions. Delay time $T$ is the the time parameter used to reveal time evolution of the exciton wavepacket $[4,10,11,13,14,17,18,24-26]$. Since different delay times are independent parameters, the time resolution of the $2 \mathrm{D}$ spectra ( $T$ delay) is in principle unrelated to the frequency resolution $\left(\omega_{t}\right.$ and $\left.\omega_{\tau}\right)$, what is never available in a classical pump-probe signal. In experiments the resolution is limited by pulse lengths and higher-order effects. The pulse overlap regions induce variations in $2 \mathrm{D}$ spectra due to mixing with various interaction sequences [12]; higher order contributions induce intensity-dependence and exciton annihilation [25].

Using the reduced density matrix formalism, the sequence of three system-field interactions can be represented as follows. At the initial time the system is in the thermal equilibrium $\hat{\rho}_{\mathrm{gg}}$. After the first interaction with a weak ultra-short pulse the state of quantum coherence 


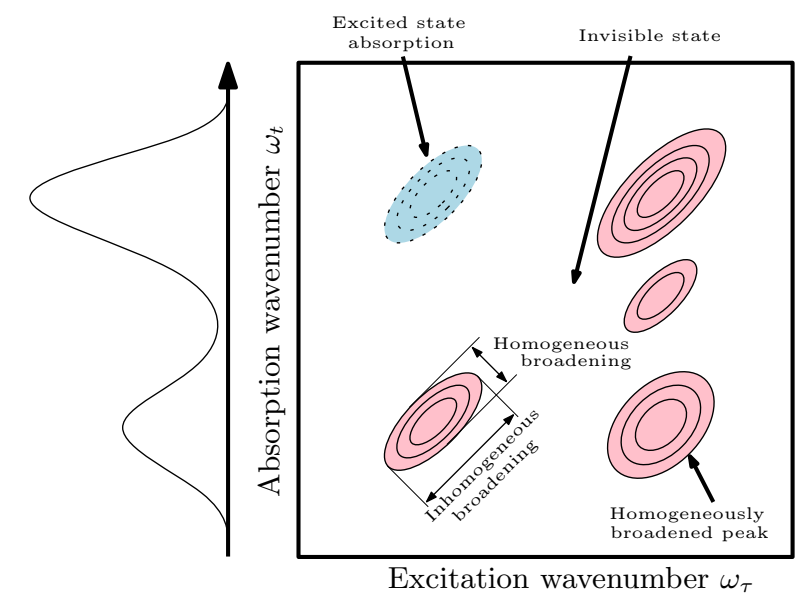

Fig. 2. Schematic representation of 2D spectra at fixed population time $T$. Diagonal elements and cross-peaks are present. The invisible state, that is not available in the absorption spectrum (left), is revealed by presence of the corresponding cross-peak. Homogeneous and inhomogeneous peak widths are separated.

$\hat{\rho}_{\mathrm{eg}}(t)$ is created. The second pulse after time $\tau$ creates a population in the ground state $\hat{\rho}_{g g}(\tau, t)$ or in the excited state $\hat{\rho}_{e e}(\tau, t)$, or a coherence in the excited state $\hat{\rho}_{e e^{\prime}}(\tau, t)\left(e \neq e^{\prime}\right)$. The third interaction creates a great variety of coherent sates and the electromagnetic field is radiated by a stimulated emission, leaving the system in the population state $\hat{\rho}_{e e}(\tau, T, t)$ or $\hat{\rho}_{g g}(\tau, T, t)$. During the time between interactions processes of dephasing and state transfer take place. As it will be shown later, a 2D optical spectroscopy enables the direct observation of dynamics of the density operator with an excellent temporal and spectral resolution.

Various representations of 2D signal are possible. The PE signal is denoted as "rephasing" since the rephasing in the system is opposite after the first and third interaction and at $\tau=t$ the inhomogeneous broadening is eliminated. In spectra it is observed as peak elongation along the main diagonal. Similarly, the signal, generated at non-PE direction $+\mathbf{k}_{1}-\mathbf{k}_{2}+\mathbf{k}_{3}$, is denoted as non-rephasing. It does not have inhomogeneous contribution eliminated and the peak lineshapes are oriented in anti-diagonal direction. The same signal would be measured in the PE direction $\mathbf{k}_{\mathrm{PE}}$ if the first and second pulses were switched (assuming $\tau<0$ ). The sum of rephasing and non-rephasing signals gives the total, pump-probe-like spectra [10, 11, 27]. Usually the real part of the total or rephasing spectra is used for interpretation.

In the experiment, both positive and negative delay time $\tau$ values can be used. For a positive delay $\tau>0$, the rephasing signal $W_{\mathrm{R}}\left(\omega_{\tau}, T, \omega_{t}\right)$ is obtained in the $\mathrm{PE}$ direction $\mathbf{k}_{\mathrm{s}}=-\mathbf{k}_{1}+\mathbf{k}_{2}+\mathbf{k}_{3}$. At the same time signal denoted as non-rephasing at non-PE direction $+\mathbf{k}_{1}-\mathbf{k}_{2}+\mathbf{k}_{3}$ is also available. The same signal would be measured in the PE direction if the first and second pulses were switched (assuming $\tau<0$ ). The sum of rephasing and non-rephasing signals gives the total pump-probe-like spectra [10, 11, 27]. Usually the real part of the total or rephasing spectra is used for interpretation.

More technical details about the experimental set-up can be found elsewhere [5, 20, 28].

\section{Theoretical background}

\subsection{Semi-classical concept of the excitation and measurement}

The core of the spectroscopy experiment is the semiclassical approximation. It denotes the separation of the incoming excitation field, the outgoing signal field, and the system. Both fields are classical (electric) fields and the system is considered as a quantum object. The whole experiment can be partitioned into two stages. In the first stage the system interacts with the incoming field. Neglecting the magnetic system properties, this interaction is described by the polarization operator and the nonlinear polarization induction by the classical electric field is described by the quantum dynamics. In the second stage the expectation value of the induced polarization becomes a source of the signal field. This stage is a problem of classical electrodynamics and is described by the Maxwell equations.

Using the density matrix formalism, the MaxwellLiouville equations describe the excitation and generation processes:

$$
\begin{aligned}
\nabla \times \nabla \times E(\mathbf{r}, t)+\frac{1}{c^{2}} \frac{\partial^{2}}{\partial t^{2}} E(\mathbf{r}, t) \\
=-\frac{4 \pi}{c^{2}} \frac{\partial^{2}}{\partial t^{2}} P(\mathbf{r}, t), \\
P(\mathbf{r}, t)=\operatorname{Tr}[\hat{P}(\mathbf{r}) \hat{\rho}(t)], \\
\frac{\partial \hat{\rho}(t)}{\partial t}=-\frac{\mathrm{i}}{\hbar}\left[\hat{H}_{\mathrm{se}}\left(E_{i}(\mathbf{r}, t)\right), \hat{\rho}(t)\right] .
\end{aligned}
$$

They read as follows: the first equation is the relationship between induced nonlinear polarization $P(\mathbf{r}, t)$ of the system and the outgoing electric field (signal $E(\mathbf{r}, t))$. This expression will be simplified reasonably assuming the phase-matching experimental geometry in Sec. 2.3. Second equation is the definition of nonlinear polarization as the trace of the polarization operator 
and the density matrix product. The third expression is equation of motion of the system density matrix driven by the excitation field $E_{i}(\mathbf{r}, t)$. Here $\hat{H}_{\text {se }}$ is the semiclassical Hamiltonian describing the quantum system under influence of the classical electric field as an external force. Thus it is obvious that having described the density matrix properly we would be able to obtain the nonlinear polarization which is the main goal of the theory of nonlinear spectroscopy.

Equations (1)-(3) are the fundamental equations of the microscopic spectroscopy formulation. They describe an arbitrary spectroscopy experiment. In the following we will separately present all the important parts of the theoretical consideration of the nonlinear spectroscopic measurement.

\subsection{Multi-wave mixing}

Incoming optical electric fields induce the dynamic polarization in the medium. In phenomenological description, if the system is nonlinear, we can expand the polarization in terms of the incoming field harmonic components as follows:

$$
\begin{aligned}
P(\omega)= & \alpha(\omega) E(\omega) \\
& +\beta\left(\omega: \omega_{1}, \omega_{2}\right) E\left(\omega_{1}\right) E\left(\omega_{2}\right) \\
& +\gamma\left(\omega: \omega_{1}, \omega_{2}, \omega_{3}\right) E\left(\omega_{1}\right) E\left(\omega_{2}\right) E\left(\omega_{3}\right) \\
& +\ldots
\end{aligned}
$$

$\alpha, \beta$, and $\gamma$ are the linear, quadratic, and the third-order susceptibilities, respectively. The optical field in principle may be given by a superposition of harmonic components. Then Eq. (4) will involve summations (integrals) over the incoming field frequencies.

Let us consider the second-order contribution

$$
P^{(2)}(\omega)=\beta\left(\omega: \omega_{1}, \omega_{2}\right) E\left(\omega_{1}\right) E\left(\omega_{2}\right) .
$$

The incoming field

$$
E(t)=\cos \left(\mathbf{k}_{0} \mathbf{r}-\omega_{0} t\right) \mathscr{E}\left[\sigma_{\omega}(t-\tau)\right]
$$

with $\mathscr{E}(x) \propto \exp \left(-x^{2} / 2\right)$ corresponds to a single pulse with wave vector $\mathbf{k}_{0}$ and central frequency $\omega_{0}$, for which $\omega_{0}=\left|\mathbf{k}_{0}\right| c$ holds. The Fourier transform of the pulse gives

$$
E(\omega) \propto \exp \left( \pm \mathbf{i} \mathbf{k}_{0} \mathbf{r}\right) \mathscr{E}\left[\left(\omega \mp \omega_{0}\right) / \sigma_{\omega}\right]
$$

In the case of the narrow-bandwidth pulse we may substitute $\omega \approx \pm \omega_{0}$ (note that $\omega$ here is a Fourier variable so it is positive and negative) into Eq. (5) and have

$$
\begin{aligned}
P^{(2)}(\omega) \approx & \overline{\mathscr{E}} \overline{\mathscr{E}}\left\{\exp \left[\mathrm{i}\left(\mathbf{k}_{0}+\mathbf{k}_{0}\right) \mathbf{r}\right] \beta\left(\omega: \omega_{0}, \omega_{0}\right)\right. \\
& +\exp \left[\mathrm{i}\left(\mathbf{k}_{0}-\mathbf{k}_{0}\right) \mathbf{r}\right] \beta\left(\omega: \omega_{0},-\omega_{0}\right) \\
& +\exp \left[\mathrm{i}\left(-\mathbf{k}_{0}+\mathbf{k}_{0}\right) \mathbf{r}\right] \beta\left(\omega:-\omega_{0}, \omega_{0}\right) \\
& \left.+\exp \left[\mathrm{i}\left(-\mathbf{k}_{0}-\mathbf{k}_{0}\right) \mathbf{r}\right] \beta\left(\omega:-\omega_{0},-\omega_{0}\right)\right\}
\end{aligned}
$$

Here $\overline{\mathscr{E}}=\mathscr{E}(0)$. We thus get that incoming fields are mixed together and the induced polarization may involve various combinations of the incoming field wave vectors. Additionally we can associate these various wave vector configurations with different nonlinear processes. For instance,

$$
\beta\left(\omega: \omega_{0}, \omega_{0}\right) \approx \delta\left(\omega-2 \omega_{0}\right) \beta\left(2 \omega_{0}: \omega_{0}, \omega_{0}\right)
$$

is the second harmonic generation and

$$
\beta\left(\omega: \omega_{0},-\omega_{0}\right) \approx \delta(\omega) \beta\left(0: \omega_{0}, \omega_{0}\right)
$$

represents the electro-optic rectification.

At higher orders of the fields we would similarly have mixing of incoming fields and would obtain a set of possible signal wave vectors. This phenomenon is called multi-wave mixing.

\subsection{Signal detection}

The nonlinear polarization induced within the sample is the source of the radiating electric field. The relationship between the laser field induced polarization (with respect to incoming fields) and the outgoing signal electric field is described by the first expression from the Maxwell-Liouville equation set (Eq. (1)). We consider that the total polarization $P(\mathbf{r}, t)$ can be expanded as a series of components according to its propagation direction:

$$
P(\mathbf{r}, t)=\sum_{n=1} \sum_{s} P_{s}^{(n)}(t) \exp \left(\mathrm{ik}_{s} \mathbf{r}-\mathrm{i} \omega_{s} t\right),
$$

where summation over index $s$ denotes summation over all possible wave vector and frequency configurations $\mathbf{k}_{s}= \pm \mathbf{k}_{1} \pm \mathbf{k}_{2} \ldots$ and $\omega_{s}= \pm \omega_{1} \pm \omega_{2} \ldots$ Since the electric field is linear in polarization, we have Eq. (1) relationship for each nonlinear component of the polarization. For a single term in Eq. (11),

$$
P(z, t)=P_{s}^{(n)}(t) \exp \left(-\mathrm{i} \omega_{s} t+\mathrm{i} k_{s} z\right),
$$


which propagates along $z$ direction, we look for a solution of the electric field (Eq. (1) in one dimension in the form of

$$
E(z, t)=E_{s}(z, t) \exp \left(-\mathrm{i} \omega_{s} t+\mathrm{i} k_{s}^{\prime} z\right)+\text { c.c. },
$$

where $k_{s}^{\prime}=\frac{\omega_{s}}{c} n\left(\omega_{s}\right)$. In Eq. (12) it is assumed that the signal, generated in the $\mathbf{k}_{s}$ direction, is parallel to the $z$ axis. Also, the rapidly oscillating part of the third-order polarization is extracted in Eq. (12) making $P_{s}^{(n)}(t)$ a slowly-varying envelope of the temporal third-order polarization dynamics. For the slowly-varying envelope the relation

$$
\left|\frac{\partial}{\partial t} P_{s}^{(n)}(t)\right| \ll\left|\omega_{s} P_{s}^{(n)}(t)\right|
$$

holds. The same approximation is valid for the electric field envelope $E(z, t)$ as well. Inserting these expressions into Eq. (1), one would obtain

$$
\mathrm{i} k_{s}^{\prime} \frac{\partial}{\partial z} E_{s}(z, t)=-2 \pi \frac{\omega_{s}^{2}}{c^{2}} P_{s}^{(n)}(t) \exp (\mathrm{i} \Delta k z),
$$

where $\Delta k \equiv k_{s}-k_{s}^{\prime}$. By integrating over the sample length from $z=0$ to $z=l$ we get

$$
\begin{aligned}
& E_{s}(l, t)= \\
& \quad \mathrm{i} \frac{2 \pi \omega_{s}}{n\left(\omega_{s}\right) c} l P_{s}^{(n)}(t) \operatorname{sinc} \frac{\Delta k l}{2} \exp \left(\mathrm{i} \frac{\Delta k l}{2}\right) .
\end{aligned}
$$

The $\operatorname{sinc}(x)=\sin (x) / x$ function is related to the phase-matching condition. If the sample size is smaller than the wavelength, $l \ll \lambda$, the signal field is radiated with an arbitrary wave vector since $\Delta k l \ll 1$ and the sinc function is equal to 1 . For macroscopic samples, $l \gg \lambda$, the sinc function becomes the Dirac delta function with respect to $\Delta k$ and the signal is generated only at $k_{s}^{\prime}=k_{s}$.

We have treated the experimental setup as onedimensional, but at this point we can make generalizations for signals in three dimensions. For microscopic samples smaller than the signal wavelength, the signal direction is not selective to wave vector of the polarization. This has implication, for instance, for single-molecule spectroscopy. For macroscopic samples, much larger than the signal wavelength, the signal contains a multiple interference from each point in the sample. The interference is positive only at the signal wave vector equal to the wave vector of the polarization.

As it was shown in a previous section the polarization can be induced only with specific wave vectors, depending on the incoming field wave vectors due to multiwave mixing. This provides a powerful utility in the experiment: while the total polarization may be radiated in many spatial directions according to multi-wave mixing represented by expansion (11), the detector can be placed in a specific position so that only one specific component of the total signal field was detected. By resolving one signal component experimentally, we can write the relationship between measured irradiance, electric field, and polarization. From equation (16) it is clear that the electric field $E_{s}(l, t)$ detected in the $\mathbf{k}_{s}$ direction is linearly proportional to the corresponding component of the induced polarization. The detected intensity is then

$$
I_{s} \propto\left|E_{s}(t)\right|^{2} \propto l^{2}\left|P_{s}^{(n)}(t)\right|^{2} .
$$

This is a very convenient result since it shows that any spectroscopic measurement can be simulated just by knowing the time dependence of the nonlinear polarization of the system. This relationship is for the homodyne detection regime where the measurement probes the signal intensity.

In the heterodyne detection scheme the detector measures a superposition of the electric field, emitted from the sample, and the external local oscillator (LO) pulse, applied in the signal direction $\mathbf{k}_{s}$. Then the detected intensity is

$$
\begin{aligned}
I_{s} & \propto\left|E_{\mathrm{LO}}(t)+P_{s}^{(n)}(t)\right|^{2} \\
& =\left|E_{\mathrm{LO}}(t)\right|^{2}+\left|P_{s}^{(n)}(t)\right|^{2}+2 \operatorname{Re}\left[E_{\mathrm{LO}}^{*}(t) P_{s}^{(n)}(t)\right] .
\end{aligned}
$$

On the rhs of this expression the first two terms can be neglected since the polarization is very weak and the LO pulse is well known and can be subtracted. The measured quantity is then the integral

$$
W(t) \equiv \operatorname{Re} \int_{-\infty}^{\infty} \mathrm{d} t\left[E_{\mathrm{LO}}^{*}(t) P_{s}^{(n)}(t)\right] .
$$

If we treat the LO as $\delta$-shaped, the measurement is $W(t)=\operatorname{Re} P_{s}^{(n)}(t)$. This result also involves the phase difference between the polarization and the LO pulse $\phi$. If the phase difference is fixed (locked), we can also probe the imaginary part of the induced polarization by taking $\phi=\pi / 2$. From Eq. $19 p$ it is then possible to extract both real and imaginary parts of the induced polarization (we will define the real and imaginary contributions later). In that case we define the heterodyne signal as the complex signal, equivalent to the induced polarization.

The above given relationships may break for certain cases. The generated signal must be considered as very 
weak compared to the incoming laser radiation so that the incoming field is not affected by the multi-wave mixing. Electric field and induced polarization dependence (Eq. (17)) also breaks for optically dense samples since the absorption effects are significant: then the polarization induction is not a separable process from field propagation.

\subsection{Induced polarization}

\subsubsection{The Liouville-space and reduced density matrix}

In this section we address the problem of Eqs. (2) and (3) and describe the quantum properties of the system. When the system constituting particles are smaller than the optical wavelength of the incident electric field, we can use the dipole approximation. Then the moleculefield interaction is assumed as a dipole-field interaction. The total Hamiltonian of the system, its environment, and the classical electric field can be written as follows:

$$
\hat{H}=\hat{H}_{\mathrm{mol}}+\hat{H}_{\mathrm{B}}+\hat{H}_{\mathrm{SB}}+\hat{H}_{\mathrm{int}}=\hat{H}_{\mathrm{mat}}-\hat{\mu} \mathbf{E}(t) .
$$

Here $\hat{H}_{\text {mol }}$ is the molecular part of the Hamiltonian, containing all degrees of freedom, which have to be included explicitly. The second term $\hat{H}_{\mathrm{B}}$ represents the reservoir (bath) causing dephasing and relaxation. It has an infinite number of degrees of freedom, they are not directly observable and will be treated approximately. $\hat{H}_{\mathrm{SB}}$ is the interaction between the system and the reservoir. These terms of the Hamiltonian constitute the material part of the system $\hat{H}_{\text {mat }}$, which does not include the optical field. The last term, $\hat{H}_{\text {int }}$, is the dipolar system-field interaction.

The dynamics of the system governed by the Hamiltonian is more conveniently described using superoperators. Superoperators are given by certain operation on operators. For example, a commutator with Hamiltonian can be written as

$$
\mathscr{L} \hat{A} \equiv[\hat{H}, \hat{A}]=\hat{H} \hat{A}-\hat{A} \hat{H} .
$$

This particular superoperator $\mathscr{L}$ is denoted as a Liouville (super)operator (Liouvillian). The density matrix evolution is expressed much more conveniently in the Liouville space, which is a direct product space of two Hilbert spaces. The regular operator, expressed as the $N \times N$ matrix in the Hilbert space, is recasted as a $N^{2}$ length vector in the Liouville space. An arbitrary superoperator can then be written as a matrix of $N^{2} \times N^{2}$ size. The operation of a superoperator on an operator then amounts to matrix and vector multiplication. The detailed introduction to Liouville-space representation of the system density matrix evolution can be found elsewhere [29].

We introduce other superoperators in an analogous way

$$
\begin{aligned}
& \mathscr{L}_{\text {mat }} \hat{A} \equiv\left[\hat{H}_{\text {mat }}, \hat{A}\right], \\
& \mathscr{L}_{\text {int }} \hat{A} \equiv[\hat{\mu}, \hat{A}] \mathbf{E}(t)=\mathscr{V} \hat{A} \mathbf{E}(t) .
\end{aligned}
$$

The equation of motion for the combined system and reservoir density matrix $\hat{\sigma}(t)$ is then given by

$$
\begin{gathered}
\frac{\partial}{\partial t} \hat{\sigma}(t)=-\frac{\mathrm{i}}{\hbar}\left[\hat{H}_{\text {mat }}-\hat{\mu} \mathbf{E}(t), \hat{\sigma}(t)\right] \\
=-\frac{\mathrm{i}}{\hbar} \mathscr{L}_{\text {mat }} \hat{\sigma}(t)+\frac{\mathrm{i}}{\hbar} \mathscr{L}_{\text {int }} \hat{\sigma}(t) .
\end{gathered}
$$

This equation is equivalent to the Schrödinger equation except that the wave function and the Hamiltonian are replaced by the density matrix and the Liouville superoperator, respectively. Thus, all formalism of quantum mechanics can be directly applied to the density matrix in the Liouville space.

However, this equation cannot be solved exactly since the number of degrees of freedom of the thermal reservoir is infinite. Instead, the reduced density matrix must be introduced. It is defined only within the system degrees of freedom. This is accomplished by averaging over the reservoir degrees of freedom. We thus define the reduced density matrix as

$$
\hat{\rho}(t)=\operatorname{Tr}_{\mathrm{B}}\{\hat{\sigma}(t)\} .
$$

Assuming that the bath is in equilibrium at all times we write the bath density matrix as

$$
\hat{\rho}_{\mathrm{B}}=\mathscr{Z}_{B}^{-1} \exp \left(-\beta \hat{H}_{\mathrm{B}}\right) \text {, }
$$

where

$$
\mathscr{Z}_{\mathrm{B}}=\sum_{\nu} \exp \left(-\beta E_{\nu}^{(\mathrm{B})}\right)
$$

is the bath partition function in terms of its eigenstates with energies $E_{\nu}^{(B)}$; and $\beta=\left(k_{\mathrm{B}} T\right)^{-1}$ is the inverse thermal energy. The density matrix of the whole system is now a direct product of the system and the bath density matrices: $\hat{\sigma}=\hat{\rho} \otimes \hat{\rho}_{B}$. This level of description is known as Born approximation.

We will be interested in the dynamics of the reduced density matrix. It is possible to derive the equation of motion for the system density matrix using an approximate perturbation theory with respect to the systemreservoir interaction [30]. The time-local equation of motion is obtained in the Markovian approximation, where the system correlation time is accepted as much 
shorter than the relevant system dynamics. For the next subsection we consider the optical properties and assume that the reservoir effects on the system density matrix can be represented by a certain time-local relaxation superoperator.

\subsubsection{Series expansion of the density matrix}

In this section we neglect the reservoir and assume $\hat{H}_{\text {mat }} \equiv \hat{H}_{\text {mol }}$ as well as $\hat{\sigma}(t) \equiv \hat{\rho}(t)$, where $\hat{H}_{\text {mol }}$ is time-independent. The field-system interaction is treated perturbatively while the material (molecular) part is the reference (see Eq. (23)). This scheme of time-dependent perturbation theory application is typical of the most spectroscopic calculations. For this type of description, the interaction picture in the Liouville space is more useful than Schrödinger or Heisenberg picture in the Hilbert space. Essentially it represents the description of the problem in the rotating frame. Evolution of the whole system can then be described as the reference. The reference molecular evolution operator in the Liouville space is defined as [29]

$$
\mathscr{U}_{\text {mol }}(t) \equiv \exp \left\{-\frac{\mathrm{i}}{\hbar} \mathscr{L}_{\text {mol }} t\right\}
$$

and any time-dependent quantity (e. g. a system density operator) can be transformed into the interaction picture as

$$
\hat{\rho}^{\mathrm{I}}(t)=\mathscr{U}_{\mathrm{mol}}^{\dagger}(t) \hat{\rho}(t),
$$

where $\mathscr{U}_{\text {mol }}^{\dagger}(t)$ denotes a hermitian conjugate operator. The transition between the Liouville and the Hilbertspace is accomplished by

$$
\begin{aligned}
& \mathscr{U}_{\mathrm{mol}}^{\dagger}(t) \hat{\rho}(t) \Rightarrow \hat{U}^{\dagger}(t) \hat{\rho}(t) \hat{U}(t) \\
& \quad=\exp \left\{\frac{\mathrm{i}}{\hbar} \hat{H}_{\mathrm{mol}} t\right\} \hat{\rho}(t) \exp \left\{-\frac{\mathrm{i}}{\hbar} \hat{H}_{\mathrm{mol}} t\right\} .
\end{aligned}
$$

Here operator $\hat{U}^{\dagger}$ acts on density operator's $b r a$ from the left and $\hat{U}$ on ket from the right. Having defined the molecular evolution operator, we are able to move to the interaction picture and calculate a time-derivative of the system density matrix using Eq. (23) for the reduced density operator:

$$
\begin{aligned}
& \frac{\partial}{\partial t} \hat{\rho}^{\mathrm{I}}(t)=\frac{\mathrm{i}}{\hbar} \mathscr{L}_{\mathrm{mol}} \mathscr{U}_{\mathrm{mol}}^{\dagger}(t) \hat{\rho}(t)+\mathscr{U}_{\mathrm{mol}}^{\dagger}(t) \frac{\partial}{\partial t} \hat{\rho}(t) \\
& \quad=\frac{\mathrm{i}}{\hbar} \mathscr{V}(t) \hat{\rho}^{\mathrm{I}}(t) E(t) .
\end{aligned}
$$

The system part $\left(\mathscr{L}_{\text {mol }}\right)$ is included into the evolution operator and the interactional term is left in the equation of motion. This expression can be formally integrated:

$$
\hat{\rho}^{\mathrm{I}}(t)=\hat{\rho}^{\mathrm{I}}\left(t_{0}\right)+\frac{\mathrm{i}}{\hbar} \int_{t_{0}}^{t} \mathrm{~d} \tau \mathscr{V}(\tau) \hat{\rho}^{\mathrm{I}}(\tau) E(\tau) .
$$

Now we move back to the Schrödinger picture. Since we treat the system density matrix as describing an equilibrium system state at initial time $t_{0}$, the molecular evolution operator does not affect it:

$$
\hat{\rho}(t)=\hat{\rho}_{\mathrm{eq}}+\frac{\mathrm{i}}{\hbar} \int_{t_{0}}^{t} \mathrm{~d} \tau \mathscr{U}_{\mathrm{mol}}(t) \mathscr{V}(\tau) \hat{\rho}(\tau) E(\tau) .
$$

By repeatedly inserting the rhs of Eq. (32) into the $\hat{\rho}(t)$ term within the integral, one would obtain an infinite series

$$
\begin{aligned}
& \hat{\rho}(t)=\hat{\rho}_{\text {eq }}+\frac{\mathrm{i}}{\hbar} \int_{t_{0}}^{t} \mathrm{~d} \tau \mathscr{U}_{\mathrm{mol}}(t) \mathscr{V}(\tau) \hat{\rho}_{\mathrm{eq}} E(\tau) \\
& +\left(\frac{\mathrm{i}}{\hbar}\right)^{2} \int_{t_{0}}^{t} \mathrm{~d} \tau \int_{t_{0}}^{\tau} \mathrm{d} \tau^{\prime} \mathscr{U}_{\mathrm{mol}}(t) \mathscr{V}(\tau) \mathscr{V}\left(\tau^{\prime}\right) \hat{\rho}_{\mathrm{eq}} E(\tau) \\
& \times E\left(\tau^{\prime}\right)+\left(\frac{\mathrm{i}}{\hbar}\right)^{3} \int_{t_{0}}^{t} \mathrm{~d} \tau \int_{t_{0}}^{\tau} \mathrm{d} \tau^{\prime} \int_{t_{0}}^{\tau^{\prime}} \mathrm{d} \tau^{\prime \prime} \mathscr{U}_{\mathrm{mol}}(t) \mathscr{V}(\tau) \\
& \times \mathscr{V}\left(\tau^{\prime}\right) \mathscr{V}\left(\tau^{\prime \prime}\right) \hat{\rho}_{\mathrm{eq}} E(\tau) E\left(\tau^{\prime}\right) E\left(\tau^{\prime \prime}\right)+\ldots
\end{aligned}
$$

\subsubsection{Linear response function}

From Eq. (2) we write for the linear polarization

$$
P^{(1)}(t)=\int_{t_{0}}^{t} \mathrm{~d} \tau S^{(1)}(t, \tau) E(\tau) .
$$

Here we have defined the linear response function $S^{(1)}(t, \tau)$. In the Liouville space it is given by

$$
S^{(1)}(t, \tau)=\frac{\mathrm{i}}{\hbar} \operatorname{Tr}\left[\hat{\mu} \mathscr{U}_{\mathrm{mol}}(t) \mathscr{V}(\tau) \hat{\rho}_{\mathrm{eq}}\right] .
$$

By defining the variable change $t_{1} \equiv t-\tau$ and sending the initial time (when system is unaffected by the interactions) to $t_{0} \rightarrow-\infty$, one would obtain

$$
P^{(1)}(t)=\int_{0}^{\infty} \mathrm{d} t_{1} S^{(1)}\left(t_{1}\right) E\left(t-t_{1}\right) .
$$


In the expression of the system response function (Eq. (35) ) superoperators are replaced by commutators of the Hilbert space:

$$
\begin{array}{r}
S^{(1)}\left(t_{1}\right)=\frac{i}{\hbar} \operatorname{Tr}\left\{\hat{\mu} \mathscr{U}_{\mathrm{mol}}\left(t_{1}\right) \mathscr{V} \hat{\rho}_{\mathrm{eq}}\right\} \\
=\frac{i}{\hbar} \operatorname{Tr}\left\{\hat{\mu} U\left(t_{1}\right)\left[\hat{\mu}, \hat{\rho}_{\mathrm{eq}}\right] U^{\dagger}\left(t_{1}\right)\right\}
\end{array}
$$

or

$$
S^{(1)}(t)=\frac{\mathrm{i}}{\hbar} \theta(t)\left[J(t)-J^{*}(t)\right]
$$

with

$$
J(t) \equiv \operatorname{Tr}\left\{\hat{\mu}(t) \hat{\mu}(0) \hat{\rho}_{\mathrm{eq}}\right\} .
$$

Expressions, which can be useful for calculations, are obtained by expanding these operator expressions in the system eigenstate basis.

\subsubsection{The third-order response function}

From Eq. (2) we obtain

$$
\begin{aligned}
& P^{(3)}(t)=\int_{t_{0}}^{t} \mathrm{~d} \tau \int_{t_{0}}^{\tau} \mathrm{d} \tau^{\prime} \int_{t_{0}}^{\tau^{\prime}} \mathrm{d} \tau^{\prime \prime} S^{(3)}\left(t, \tau, \tau^{\prime}, \tau^{\prime \prime}\right) \\
& \times E(\tau) E\left(\tau^{\prime}\right) E\left(\tau^{\prime \prime}\right) .
\end{aligned}
$$

Here we have defined the third-order system response function $S^{(3)}\left(t, \tau, \tau^{\prime}, \tau^{\prime \prime}\right)$. In the Liouville space it is given by

$$
\begin{aligned}
& S^{(3)}\left(t, \tau, \tau^{\prime}, \tau^{\prime \prime}\right) \\
& \quad=\left(\frac{\mathrm{i}}{\hbar}\right)^{3} \operatorname{Tr}\left[\hat{\mu} \mathscr{U}_{\mathrm{mol}}(t) \mathscr{V}(\tau) \mathscr{V}\left(\tau^{\prime}\right) \mathscr{V}\left(\tau^{\prime \prime}\right) \hat{\rho}_{\mathrm{eq}}\right] .
\end{aligned}
$$

By committing a variable change $t_{3} \equiv t-\tau, t_{2} \equiv \tau-\tau^{\prime}$, $t_{1} \equiv \tau^{\prime}-\tau^{\prime \prime}$ and sending the initial time to $t_{0} \rightarrow-\infty$, we obtain

$$
\begin{aligned}
& P^{(3)}(t)=\int_{0}^{\infty} \mathrm{d} t_{3} \int_{0}^{\infty} \mathrm{d} t_{2} \int_{0}^{\infty} \mathrm{d} t_{1} S^{(3)}\left(t_{3}, t_{2}, t_{1}\right) \\
& \times E\left(t-t_{3}\right) E\left(t-t_{3}-t_{2}\right) E\left(t-t_{3}-t_{2}-t_{1}\right) .
\end{aligned}
$$

This formula is the most useful expression of the thirdorder polarization. It was obtained by applying a perturbative scheme upon the equation of motion of the system density operator. It is a convolution of the thirdorder response function and a product of electric field functions. From Eq. (42) it is evident that the thirdorder polarization is a function of $t$, but, however, it is also dependent on $T$ and $\tau$ via the electric field components representing laser pulse configuration. Now we must find an efficient way to calculate the system response function and express the multiplication of electric fields in a convenient form.

In the expression of the system response function (Eq. (41)) the superoperators are replaced by commutators of the Hilbert space:

$$
\begin{aligned}
& S^{(3)}\left(t_{3}, t_{2}, t_{1}\right) \\
& =\left(\frac{\mathrm{i}}{\hbar}\right)^{3} \operatorname{Tr}\left\{\hat{\mu} \mathscr{U}_{\mathrm{mol}}\left(t_{3}\right) \mathscr{V} \hat{\mu} \mathscr{U}_{\mathrm{mol}}\left(t_{2}\right) \mathscr{V} \mathscr{U}_{\mathrm{mol}}\left(t_{1}\right) \hat{\rho}_{\mathrm{eq}}\right\} \\
& =\left(\frac{\mathrm{i}}{\hbar}\right)^{3} \operatorname{Tr}\left\{\hat { \mu } U ( t _ { 3 } ) \left[\hat{\mu}, U\left(t_{2}\right)\left[\hat{\mu}, U\left(t_{1}\right)\left[\hat{\mu}, \hat{\rho}_{\mathrm{eq}}\right]\right.\right.\right. \\
& \left.\left.\left.\times U^{\dagger}\left(t_{1}\right)\right] U^{\dagger}\left(t_{2}\right)\right] U^{\dagger}\left(t_{3}\right)\right\} .
\end{aligned}
$$

Expanding the commutators we get

$$
\begin{aligned}
& S^{(3)}\left(t_{3}, t_{2}, t_{1}\right)=\left(\frac{\mathrm{i}}{\hbar}\right)^{3} \theta\left(t_{1}\right) \theta\left(t_{2}\right) \theta\left(t_{3}\right) \\
& \quad \times \sum_{\alpha=1}^{4}\left[R_{\alpha}\left(t_{3}, t_{2}, t_{1}\right)-R_{\alpha}^{\star}\left(t_{3}, t_{2}, t_{1}\right)\right],
\end{aligned}
$$

where

$$
\begin{aligned}
& R_{1}\left(t_{3}, t_{2}, t_{1}\right) \\
& =\operatorname{Tr}\left\{\hat{\mu}\left(t_{1}\right) \hat{\mu}\left(t_{1}+t_{2}\right) \hat{\mu}\left(t_{1}+t_{2}+t_{3}\right) \hat{\mu}(0) \hat{\rho}_{\mathrm{eq}}\right\}, \\
& R_{2}\left(t_{3}, t_{2}, t_{1}\right) \\
& =\operatorname{Tr}\left\{\hat{\mu}(0) \hat{\mu}\left(t_{1}+t_{2}\right) \hat{\mu}\left(t_{1}+t_{2}+t_{3}\right) \hat{\mu}\left(t_{1}\right) \hat{\rho}_{\mathrm{eq}}\right\}, \\
& R_{3}\left(t_{3}, t_{2}, t_{1}\right) \\
& =\operatorname{Tr}\left\{\hat{\mu}(0) \hat{\mu}\left(t_{1}\right) \hat{\mu}\left(t_{1}+t_{2}+t_{3}\right) \hat{\mu}\left(t_{1}+t_{2}\right) \hat{\rho}_{\mathrm{eq}}\right\}, \\
& R_{4}\left(t_{3}, t_{2}, t_{1}\right) \\
& =\operatorname{Tr}\left\{\hat{\mu}\left(t_{1}+t_{2}+t_{3}\right) \hat{\mu}\left(t_{1}+t_{2}\right) \hat{\mu}\left(t_{1}\right) \hat{\mu}(0) \hat{\rho}_{\mathrm{eq}}\right\} .
\end{aligned}
$$

Heaviside functions in Eq. (44) emphasize the principle of causality: as it is seen in Eq. (42), the third-order polarization at time $t$ depends on the electric field of earlier times. In other words, the electric field in the past (a cause) determines the polarization in the future, so if any of system response function arguments is negative, the function must be zero. Also one can notice that the total system response function is always real. It is clear from the experiment, since the polarization is a measurable quantity and has a corresponding hermitian 

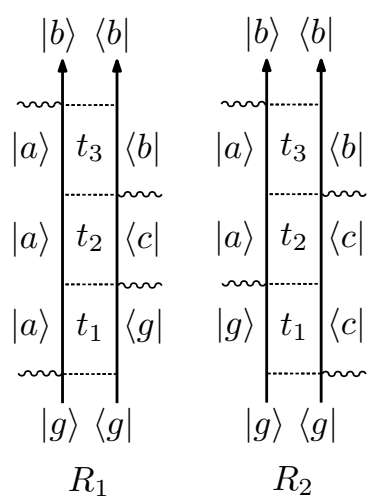

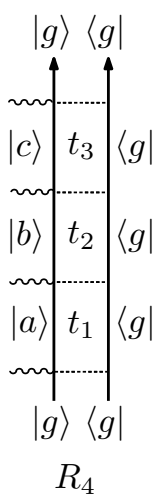

$R_{3}$

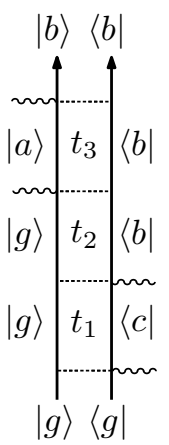

where evolution of the material part is decomposed into the molecular and dissipational parts given by $\mathscr{L}_{\text {mol } \ldots} \equiv\left[\hat{H}_{\text {mol }}, \ldots\right]$ and $\mathscr{D}$, respectively, and the interaction with the field is $\mathscr{L}_{\text {int }}$. The interaction part depends on the electric field. At point $\mathbf{r}$ of the space

$$
E(t)=\frac{1}{2} E_{0} \sum_{i=j}^{3} \mathscr{E}_{j}\left(t-\tau_{j}\right) \mathrm{e}^{\mathrm{i} \mathbf{k}_{j} \mathbf{r}-\mathrm{i} \omega_{j}\left(t-\tau_{j}\right)}+\text { c.c. },
$$

Fig. 3. Double-sided Feynman diagrams, corresponding to the components of nonlinear response function in Eqs. (46)-(49) for a general multi-level system, where $|a\rangle-|c\rangle$ stand for energy states; $|g\rangle$ is ground state.

quantum mechanical operator, the average of which is always real.

Because of the cyclic nature of the trace operation (it is valid to commit a cyclic permutation of operators inside trace brackets), chronologically ordered dipole moment operators can act upon the density operator from either side, i. e. they act upon a bra or ket of the density operator. These sequences for Eqs. (46)-(49) are conveniently expressed schematically using the abstract double-sided Feynman diagrams [7, 23, 29, 31] (Fig. 3). Vertical arrows denote the time direction; variables $t_{1}$, $t_{2}$, and $t_{3}$ are the time intervals between two successive interactions of the dipole operator and the system density operator the (electric field and the system); horizontal lines show the side of the dipole operator action upon the density operator. A ket and a bra during the same time interval $(|\alpha\rangle\langle\beta|)$ denote a state of coherence $(\alpha \neq \beta)$ or population $(\alpha=\beta)$ corresponding to an element of the system density matrix being affected.

However, the whole system response depends on the electric field (i.e. the direction and time of incident pulses). So it is possible to express all permutations of interaction orders and directions in the excitonic basis by double-sided diagrams as well. It will be shown in Sec. 4.2.

\subsubsection{A non-perturbative propagation of the density matrix}

The induced polarization can also be calculated nonperturbatively. We have the equation of motion of the time dependent density matrix with the optical field included explicitly as

$$
\frac{\partial}{\partial t} \hat{\rho}(t)=-\frac{\mathrm{i}}{\hbar}\left(\mathscr{L}_{\mathrm{mol}}-\mathrm{i} \mathscr{D}+\mathscr{L}_{\mathrm{int}}\right) \hat{\rho}(t)
$$

where $\mathscr{E}_{j}$ is a temporal Gaussian envelope function of $j$ th laser pulse and $\tau_{1}, \tau_{2}$, and $\tau_{3}$ are the central times of each pulse (see experimental scheme in Fig. (11)).

To select specific polarization configuration, corresponding to certain signal wave vector, one needs to distinguish different density matrices with different wave vectors. This is easily accomplished when the incoming pulses do not overlap. For instance, consider the first interaction. To distinguish $+\mathbf{k}_{1}$ and $-\mathbf{k}_{1}$ density matrices the former must include only multiplication by the dipole operator from the left, while the latter is obtained by multiplying the dipole operator from the right. We then have

$$
\begin{aligned}
\frac{\partial}{\partial t} \hat{\rho}_{+}^{(1)}(t)= & -\frac{\mathrm{i}}{\hbar}\left(\mathscr{L}_{\text {mol }}-\mathrm{i} \mathscr{D}\right) \hat{\rho}_{+}^{(1)}(t) \\
& +\frac{\mathrm{i}}{\hbar} \hat{\mu} \hat{\rho}^{(0)}(t) \mathscr{E}_{1}\left(t-\tau_{1}\right) \mathrm{e}^{-\mathrm{i} \omega_{1}\left(t-\tau_{1}\right)}
\end{aligned}
$$

and

$$
\begin{aligned}
\frac{\partial}{\partial t} \hat{\rho}_{-}^{(1)}(t)= & -\frac{\mathrm{i}}{\hbar}\left(\mathscr{L}_{\mathrm{mol}}-\mathrm{i} \mathscr{D}\right) \hat{\rho}_{-}^{(1)}(t) \\
& +\frac{\mathrm{i}}{\hbar} \hat{\rho}^{(0)}(t) \hat{\mu} \mathscr{E}_{1}\left(t-\tau_{1}\right) \mathrm{e}^{+\mathrm{i} \omega_{1}\left(t-\tau_{1}\right)} .
\end{aligned}
$$

Note, however, that $\left[\hat{\rho}_{+}^{(1)}\right]_{e g}=\left[\hat{\rho}_{-}^{(1)}\right]_{g e}^{*}$, so it is enough to solve one equation. For the second interaction we would have four possible terms corresponding to $+\mathbf{k}_{1}+$ $\mathbf{k}_{2},+\mathbf{k}_{1}-\mathbf{k}_{2},-\mathbf{k}_{1}+\mathbf{k}_{2}$, and $-\mathbf{k}_{1}-\mathbf{k}_{2}$. Relevant equations are as follows:

$$
\begin{aligned}
\frac{\partial}{\partial t} \hat{\rho}_{++}^{(2)}(t)= & -\frac{\mathrm{i}}{\hbar}\left(\mathscr{L}_{\mathrm{mol}}-\mathrm{i} \mathscr{D}\right) \hat{\rho}_{++}^{(2)}(t) \\
& +\frac{\mathrm{i}}{\hbar} \hat{\mu} \hat{\rho}_{+}^{(1)}(t) \mathscr{E}_{2}\left(t-\tau_{2}\right) \mathrm{e}^{-\mathrm{i} \omega_{2}\left(t-\tau_{2}\right)} \\
\frac{\partial}{\partial t} \hat{\rho}_{-+}^{(2)}(t)= & -\frac{\mathrm{i}}{\hbar}\left(\mathscr{L}_{\mathrm{mol}}-\mathrm{i} \mathscr{D}\right) \hat{\rho}_{-+}^{(2)}(t) \\
& +\frac{\mathrm{i}}{\hbar} \hat{\mu}\left[\hat{\rho}_{+}^{(1)}(t)\right]^{\dagger} \mathscr{E}_{2}\left(t-\tau_{2}\right) \mathrm{e}^{-\mathrm{i} \omega_{2}\left(t-\tau_{2}\right)}
\end{aligned}
$$


and the other two are just complex conjugated. For the third interaction we can choose the photon echo configuration $-\mathbf{k}_{1}+\mathbf{k}_{2}+\mathbf{k}_{3}$, where we have equation:

$$
\begin{aligned}
\frac{\partial}{\partial t} \hat{\rho}_{-++}^{(3)}(t)= & -\frac{\mathrm{i}}{\hbar}\left(\mathscr{L}_{\mathrm{mol}}-\mathrm{i} \mathscr{D}\right) \hat{\rho}_{-++}^{(3)}(t) \\
& +\frac{\mathrm{i}}{\hbar} \hat{\mu} \hat{\rho}_{-+}^{(2)}(t) \mathscr{E}_{3}\left(t-\tau_{3}\right) \mathrm{e}^{-\mathrm{i} \omega_{3}\left(t-\tau_{3}\right)} .
\end{aligned}
$$

Solution for these equations may be performed in an arbitrary basis set, however, if the Schrödinger equation for the system can be solved and the dissipational superoperator can be calculated, the solution is much simpler in the eigenstate basis. The photon echo polarization is then given by the trace of the polarization operator with the relevant system density matrix:

$$
P(\tau, T, t)=\sum_{\alpha \beta} \mu_{\alpha \beta}\left[\hat{\rho}_{-++}^{(3)}\right]_{\beta \alpha},
$$

here $\alpha$ and $\beta$ represent all possible eigenstates (the ground state, one-, and two- exciton states). Specific resonant manifolds of states can be selected in the eigenstate basis set by considering only resonant interactions. Then, for instance, in Eq. (49) we select only system states which have the energy splitting close to the optical frequency. If we label the ground state as $g$ with energy 0 , then only the following solution is necessary:

$$
\begin{aligned}
\frac{\partial}{\partial t}\left[\hat{\rho}_{+}^{(1)}\right]_{e g}= & -\mathrm{i} \omega_{e g}\left[\hat{\rho}_{+}^{(1)}\right]_{e g}-\mathscr{D}_{e g, e^{\prime} g}\left[\hat{\rho}_{+}^{(1)}\right]_{e^{\prime} g} \\
& +\frac{\mathrm{i}}{\hbar} \mu_{e g} \mathscr{E}_{1}\left(t-\tau_{1}\right) \mathrm{e}^{-\mathrm{i} \omega_{1}\left(t-\tau_{1}\right)},
\end{aligned}
$$

with $\omega_{e g} \approx \omega_{1}$. Note that the zero-order ground state density matrix $\left[\hat{\rho}^{(0)}\right]_{g g} \equiv 1$.

The decomposition presented above is equivalent to the third-order perturbative expansion presented in previous sections. Another type of decomposition is capable to select the desired signal. It is based on phase cycling. Consider the optical field in Eq. (50). The $\mathbf{k}_{j} \mathbf{r}$ term in the exponent is the phase of the field. Consider that instead of one system we have a lattice of systems along the incoming field propagation direction. Then at each site of the lattice, $k$, the phases will be $\mathbf{k}_{j} \mathbf{r}_{k}$. Having $N$ sites in the lattice separated by lattice constant $a$ we can perform a Fourier transform of a quantity, which depends on $k$ and have a set of wave vectors $k^{\prime}$ associated to the lattice. The maximum lattice wave vector is $k_{N}^{\prime}=2 \pi / a$ and the step between these wave vectors is $\Delta k^{\prime}=2 \pi /(N a)$. If $a=2 \pi /\left(N k_{j}\right)$, we would get $\Delta k^{\prime}=k_{j}$. If we then have a quantity as a function of the lattice site, its discrete Fourier transform is given as a function of $k_{j}, 2 k_{j}$, $3 k_{j}, \ldots$ The symmetries of Fourier transform imply that the amplitude at $(N-1) k_{j}$ is equivalent to $-k_{j}$ and at $(N-2) k_{j}$ to $(N-2) k_{j} \equiv-2 k_{j}$. Calculating the quantity at $N$ lattice sites thus allows to decompose it into components corresponding to phases $\exp (-\mathrm{i} n k r)$ with $n=-N / 2,-N / 2+1, \ldots 0 \ldots+N / 2-1$ for even $N$ (odd $N$ can be incorporated similarly).

Such decomposition can be performed for all three incoming fields as a three-dimensional lattice, and the induced polarization can be calculated for any combination of the incoming field wave vectors. For instance, for configuration $\mathbf{k}_{\mathrm{s}}=-\mathbf{k}_{1}+\mathbf{k}_{2}+\mathbf{k}_{3}$ it is sufficient to take $N=4$ and this configuration can be extracted by a discrete Fourier transform of the calculated signal at various lattice sites. Different lattice sites correspond to different phases of the incoming fields, thus, this procedure is called phase cycling (originally without invoking the lattice concept). The convenience of this spatial Fourier transform is as follows: for a specific $N$ the calculated signal exactly extracts a particular signal wave vector up to $N$ th order in the field. Higher orders in the field are mixed. Thus, by taking large $N$ this procedure allows us to calculate the signal to a specified order in the field. For instance, the third order in $\mathbf{k}_{\mathrm{s}}=-\mathbf{k}_{1}+\mathbf{k}_{2}+\mathbf{k}_{3}$ signal direction is the lowest order which contributes. 5th, 7th, etc. order in the field can therefore be calculated by the phase cycling. In the perturbative scheme this would require calculation of higher order perturbation series expressions which unfortunately scale very unfavorably with the order in the field. Non-perturbative approach [25] has been used to account for higher than third-order effects in the nonlinear signals.

\subsection{Overlapping electric fields in perturbative expansion}

Let us consider the product of the total incoming field (from Eq. (42))

$E\left(\mathbf{r}, t-t_{3}-t_{2}-t_{1}\right) E\left(\mathbf{r}, t-t_{3}-t_{2}\right) E\left(\mathbf{r}, t-t_{3}\right)$. 
For convenience we can separate a spatial phase factor $\Phi_{j}=\mathbf{k}_{j} \mathbf{r}$ from the time-dependent factors and identify the interaction sequence:

$$
\begin{array}{r}
E_{j}\left(\mathbf{r}, t-t_{3}-t_{2}-t_{1}\right) \equiv E_{j}^{[1]} \mathrm{e}^{\mathrm{i} \Phi_{j}}+\bar{E}_{j}^{[1]} \mathrm{e}^{-\mathrm{i} \Phi_{j}}, \\
E_{j}\left(\mathbf{r}, t-t_{3}-t_{2}\right) \equiv E_{j}^{[2]} \mathrm{e}^{\mathrm{i} \Phi_{j}}+\bar{E}_{j}^{[2]} \mathrm{e}^{-\mathrm{i} \Phi_{j}}, \\
E_{j}\left(\mathbf{r}, t-t_{3}\right) \equiv E_{j}^{[3]} \mathrm{e}^{\mathrm{i} \Phi_{j}}+\bar{E}_{j}^{[3]} \mathrm{e}^{-\mathrm{i} \Phi_{j}},
\end{array}
$$

where now $E_{j}^{[1]}$ denotes a forward-propagating pulse which interacts first with the system, $E_{j}^{[2]}$ stands for the second-interacting pulse, and $E_{j}^{[3]}$ for the third. $\bar{E}_{j}^{[m]}=$ $E_{j}^{[m] *}$ indicates the backward propagation (conjugate part); here both $j, m=1,2,3$. The expressions for $E_{j}^{[m]}$ are as follows:

$$
\begin{aligned}
E_{j}^{[1]} \equiv & \mathscr{E}_{j}\left(t-t_{3}-t_{2}-t_{1}-\tau_{j}\right) \\
& \times \mathrm{e}^{-\mathrm{i} \omega_{j}\left(t-t_{3}-t_{2}-t_{1}-\tau_{j}\right)} \mathrm{e}^{\mathrm{i} \phi_{j}}, \\
E_{j}^{[2]} \equiv & \mathscr{E}_{j}\left(t-t_{3}-t_{2}-\tau_{j}\right) \mathrm{e}^{-\mathrm{i} \omega_{j}\left(t-t_{3}-t_{2}-\tau_{j}\right)} \mathrm{e}^{\mathrm{i} \phi_{j}}, \\
E_{j}^{[3]} \equiv & \mathscr{E}_{j}\left(t-t_{3}-\tau_{j}\right) \mathrm{e}^{-\mathrm{i} \omega_{j}\left(t-t_{3}-\tau_{j}\right)} \mathrm{e}^{\mathrm{i} \phi_{j}} .
\end{aligned}
$$

Since we are considering a response in the direction $-\mathbf{k}_{1}+\mathbf{k}_{2}+\mathbf{k}_{3}$, we can neglect terms which have spatial phase factors different from $-\Phi_{1}+\Phi_{2}+\Phi_{3}$ after substituting Eqs. (59) into Eq. (42). Assuming all electric field pulses being of the same frequency $\left(\omega_{0} \equiv \omega_{1}=\right.$ $\left.\omega_{2}=\omega_{3}\right)$ and phase not tuned $\left(\phi_{1}=\phi_{2}=\phi_{3}=0\right)$, the product of the total incoming field (Eq. (58)) is

$$
\begin{aligned}
& {\left[\bar{E}_{1}^{[1]} E_{2}^{[2]} E_{3}^{[3]}+\bar{E}_{1}^{[1]} E_{3}^{[2]} E_{2}^{[3]}\right) \mathrm{e}^{\mathrm{i} \omega_{0}\left(t_{3}-t_{1}\right)} } \\
\times & \left(E_{2}^{[1]} \bar{E}_{1}^{[2]} E_{3}^{[3]}+E_{3}^{[1]} \bar{E}_{1}^{[2]} E_{2}^{[3]}\right) \mathrm{e}^{\mathrm{i} \omega_{0}\left(t_{3}+t_{1}\right)} \\
\times & \left.\left(E_{2}^{[1]} E_{3}^{[2]} \bar{E}_{1}^{[3]}+E_{3}^{[1]} E_{2}^{[2]} E_{1}^{[3]}\right) \mathrm{e}^{\mathrm{i} \omega_{0}\left(t_{3}+2 t_{2}+t_{1}\right)}\right] \\
\times & \mathrm{e}^{-\mathrm{i} \omega_{0}(t-\tau)} .
\end{aligned}
$$

Terms in this product lie in three groups according to their phase factors. They are $\mathrm{e}^{\mathrm{i} \omega_{0}\left(t_{3}-t_{1}\right)}, \mathrm{e}^{\mathrm{i} \omega_{0}\left(t_{3}+t_{1}\right)}$, and $\mathrm{e}^{\mathrm{i} \omega_{0}\left(t_{3}+2 t_{2}+t_{1}\right)}$. As it will be shown later, system response function can be decomposed into components having opposite phase factors. In a multiplication of the system response function and electric field product some of these phase factors will cancel making that term slowly-varying, others will be modulated by the sum frequencies. Integration over time (Eq. (42)) makes the latter ones negligible compared to integrals of slowly-varying terms. This elimination is known as the rotating-wave approximation (RWA).

\subsection{Two-dimensional photon echo signal}

The third-order polarization is the main quantity under consideration in theory of nonlinear response of bulk isotropic samples. One of the most advanced experimental set-ups is presented in the scheme in Fig. 11: here the polarization is induced by three incoming laser fields. According to the scheme we can consider the set of signal wave vectors representing all permutations of incoming field wave vectors $\mathbf{k}_{s}= \pm \mathbf{k}_{1} \pm \mathbf{k}_{2} \pm \mathbf{k}_{3}$ and, correspondingly, frequencies $\omega_{s}= \pm \omega_{1} \pm \omega_{2} \pm \omega_{3}$. In this scheme the signal may be defined as the function of the delay times between the laser pulses, $W(\tau, T, t) \equiv$ $P^{(3)}(\tau, T, t)$.

A two-dimensional photon echo spectrum is obtained by applying 2D Fourier transform of polarization $P^{(3)}(\tau, T, t)$, detected in $-\mathbf{k}_{1}+\mathbf{k}_{2}+\mathbf{k}_{3}$ direction, over time variables $\tau$ and $t$. As it was mentioned in the introduction, separation of the total signal to the rephasing and non-rephasing signals is possible according to the range of delay time $\tau$. For the rephasing signal $(\tau>0)$,

$$
\begin{aligned}
& W_{\mathrm{R}}\left(\omega_{\tau}, T, \omega_{t}\right) \\
& =\int_{0}^{\infty} \mathrm{d} \tau \mathrm{e}^{-\mathrm{i} \omega_{\tau} \tau} \int_{0}^{\infty} \mathrm{d} t \mathrm{e}^{-\mathrm{i} \omega_{t} t} P_{-\mathbf{k}_{1}+\mathbf{k}_{2}+\mathbf{k}_{3}}^{(3)}(\tau, T, t) .
\end{aligned}
$$

In the scheme of the non-rephasing signal generation, first two pulses are exchanged, but the detection takes place in $-\mathbf{k}_{1}+\mathbf{k}_{2}+\mathbf{k}_{3}$ direction. The same signal would be detected in $+\mathbf{k}_{1}-\mathbf{k}_{2}+\mathbf{k}_{3}$ experimental direction if $\tau$ was positive. Just its spectral elements would lie in the opposite plane of $\omega_{\tau}$ with respect to rephasing spectrum:

$$
\begin{aligned}
& W_{\mathrm{NR}}\left(-\omega_{\tau}, T, \omega_{t}\right) \\
& =\int_{0}^{\infty} \mathrm{d} \tau \mathrm{e}^{\mathrm{i} \omega_{\tau} \tau} \int_{0}^{\infty} \mathrm{d} t \mathrm{e}^{-\mathrm{i} \omega_{t} t} P_{+\mathbf{k}_{1}-\mathbf{k}_{2}+\mathbf{k}_{3}}^{(3)}(\tau, T, t) .
\end{aligned}
$$

The integration over $t$ is carried out only in the positive axis since the third-order response is not created before the third laser pulse.

\section{Response of basic quantum systems}

Here we present the least sophisticated systems that can be analysed using methods of 2D spectroscopy. Purely for the demonstrational purposes some features 
of this technique can be highlighted in an elegant way and analytic expressions of 2D and absorption spectra can be obtained for some basic quantum systems.

This section is organized as follows. In Section 3.1 an isolated two-level system is considered. The phonon bath is taken into account as the dephasing of the system response. In Section 3.2 the dephasing is expressed using an energy-gap correlation function and the lineshape function for a two-level system is introduced. Depending on the model of a phonon-bath motion, various approximations are presented. In Section 3.3 a model of the two-level system is appended by a third overtone state which is a basis of an anharmonic oscillator system. Analytic expressions of system response are presented using system-bath interaction description as for a two-level atom. And, finally, in Section 3.4, the response of a general multi-level system is derived assuming the second-order cumulant expansion of systembath interaction.

\subsection{Two-level atom}

In this part we consider an ideal quantum system of two energy levels: the ground state $|g\rangle$ and the excited state $|e\rangle$. This model effectively represents an isolated resonant transition of an atom. The total Hamiltonian in the system eigenstate basis consists of the material part and the coupling with electric field

$$
\hat{H}=\varepsilon_{g}|g\rangle\left\langle g\left|+\varepsilon_{e}\right| e\right\rangle\langle e|-\hat{\mu} E(t) .
$$

The evolution superoperator, corresponding to the material part of the Hamiltonian, acting upon the dipole moment operator $\hat{\mu}=\mu_{g e}|g\rangle\left\langle e\left|+\mu_{e g}\right| e\right\rangle\langle g|$ also possesses the phenomenologically included dephasing term $\gamma$.

$$
\mathscr{U}_{\text {mol }}^{\dagger}\left(t_{1}\right) \hat{\mu}=\theta\left(t_{1}\right) \exp \left\{\mathrm{i} \omega_{e g} t_{1}-\gamma t_{1}\right\}|e\rangle\langle g|+\text { h.c. }
$$

Inserting this into the definition of the linear response function (Eq. (37)) together with $\hat{\rho}_{e q}=|g\rangle\langle g|$ results in

$$
S^{(1)}\left(t_{1}\right)=-\frac{2}{\hbar} \theta\left(t_{1}\right)\left|\mu_{g e}\right|^{2} \mathrm{e}^{-\gamma t_{1}} \sin \left(\omega_{e g} t_{1}\right) .
$$

The Fourier-transformed linear response function is

$$
\begin{aligned}
& S^{(1)}\left(\omega_{1}\right)=\frac{\mathrm{i}}{\hbar}\left|\mu_{e g}\right|^{2} \\
& \times\left[\frac{1}{\gamma+\mathrm{i}\left(\omega_{1}-\omega_{e g}\right)}-\frac{1}{\gamma+\mathrm{i}\left(\omega_{1}+\omega_{e g}\right)}\right] .
\end{aligned}
$$

For an isolated two-level system it is a Lorentzianshaped function, centred at $\omega_{e g}$.
In the third-order photon echo response function we have only two contributions, ground state bleaching and stimulated emission:

$$
\begin{aligned}
S^{\mathrm{GSB}}\left(t_{3}, t_{2}, t_{1}\right) & \equiv R_{2}\left(t_{3}, t_{2}, t_{1}\right) \\
& =\mu^{4} \mathrm{e}^{\mathrm{i} \omega_{e g}\left(t_{1}-t_{3}\right)-\gamma\left(t_{1}+t_{3}\right)}, \\
S^{\mathrm{SE}}\left(t_{3}, t_{2}, t_{1}\right) & \equiv R_{3}\left(t_{3}, t_{2}, t_{1}\right) \\
& =\mu^{4} \mathrm{e}^{\mathrm{i} \omega_{e g}\left(t_{1}-t_{3}\right)-\gamma\left(t_{1}+t_{3}\right)},
\end{aligned}
$$

while $R_{1}\left(t_{3}, t_{2}, t_{1}\right)$ and $R_{4}\left(t_{3}, t_{2}, t_{1}\right)$ contributions are zero. To obtain an analytic expression of the $2 \mathrm{D}$ spectrum of the two-level atom, we consider $\delta$-shaped laser pulses. In this regime (impulsive limit), 2D spectrum is a Fourier image of the system response function (third order polarization equals the system response function):

$$
\begin{aligned}
W\left(\omega_{\tau}, T, \omega_{t}\right)= & \int_{-\infty}^{\infty} \mathrm{d} t_{3} \mathrm{e}^{-\mathrm{i} \omega_{\tau} \tau} \int_{-\infty}^{\infty} \mathrm{d} t_{1} \mathrm{e}^{-\mathrm{i} \omega_{t} t} \\
& \times S^{(3)}\left(t \equiv t_{3}, T \equiv t_{2}, \tau \equiv t_{1}\right)
\end{aligned}
$$

and

$$
\begin{aligned}
& S^{(3)}\left(t_{3}, t_{2}, t_{1}\right)=\left(\frac{i}{\hbar}\right)^{3} \theta\left(t_{1}\right) \theta\left(t_{2}\right) \theta\left(t_{3}\right) \mu^{4} \\
& \times\left[R_{2}\left(t_{3}, t_{2}, t_{1}\right)+R_{3}\left(t_{3}, t_{2}, t_{1}\right)\right] \\
& =2\left(\frac{i}{\hbar}\right)^{3} \theta\left(t_{1}\right) \theta\left(t_{2}\right) \theta\left(t_{3}\right) \mu^{4} \mathrm{e}^{\mathrm{i} \omega_{e g}\left(t_{1}-t_{3}\right)-\gamma\left(t_{1}+t_{3}\right)} .
\end{aligned}
$$

By applying Fourier transform and separating rephasing $(\tau>0)$ and non-rephasing $(\tau<0)$ parts, we obtain

$$
\begin{aligned}
& W_{\mathrm{R}}\left(\omega_{\tau}, \omega_{t}\right) \\
& =2\left(\frac{i}{\hbar}\right)^{3} \mu^{4} \frac{1}{\gamma-\mathrm{i}\left(\omega_{t}-\omega_{e g}\right)} \cdot \frac{1}{\gamma+\mathrm{i}\left(\omega_{\tau}-\omega_{e g}\right)}, \\
& W_{\mathrm{NR}}\left(\omega_{\tau}, \omega_{t}\right) \\
& =2\left(\frac{i}{\hbar}\right)^{3} \mu^{4} \frac{1}{\gamma-\mathrm{i}\left(\omega_{t}-\omega_{e g}\right)} \cdot \frac{1}{\gamma-\mathrm{i}\left(\omega_{\tau}-\omega_{e g}\right)} .
\end{aligned}
$$

The calculated 2D spectra of the two-level system are depicted in Fig. 4. Note that non-rephasing 2D spectra of the real and imaginary parts are just mirrored images of the rephasing spectra with respect to $\omega_{\tau}$ axis. In the absence of the inhomogeneous broadening both real part rephasing and non-rephasing spectra are 


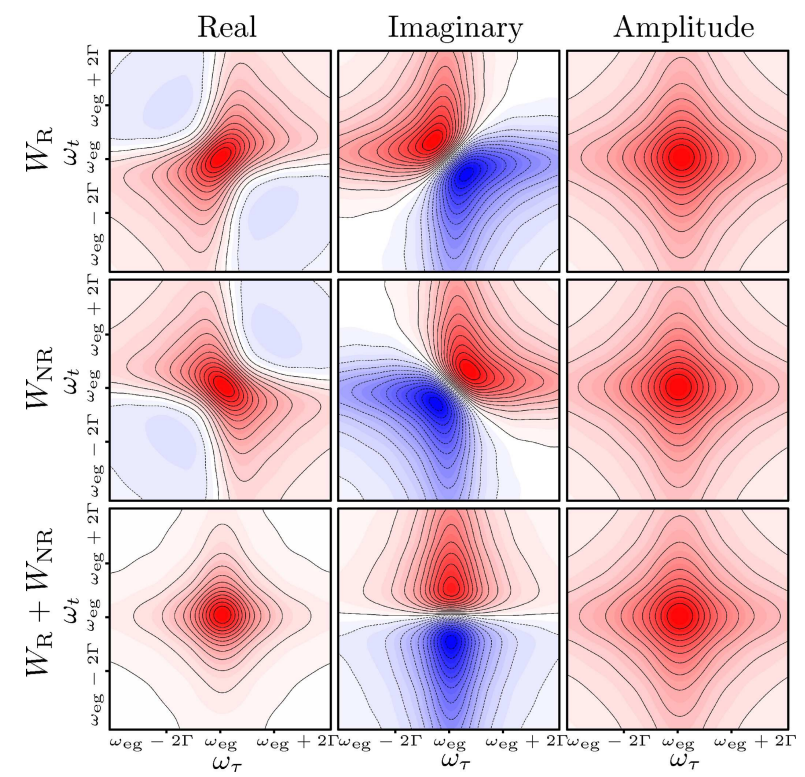

Fig. 4. Calculated rephasing, non-rephasing, and sum spectra for the real (absorptive signal) and imaginary (dispersive signal) parts and signal amplitude of a single two-level system (isolated two-level atom).
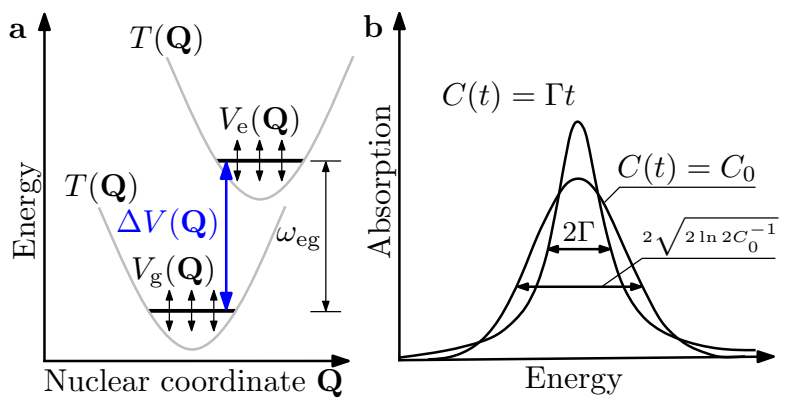

Fig. 5. (a) Scheme of the two-level molecule under consideration with elements of molecular Hamiltonian indicated; (b) lineshapes of absorption peaks of two-level molecule in homogeneous and inhomogeneous limits.

Lorentzian-shaped along the main diagonal with $2 \gamma$ for the FWHM (Fig. 5b).

\subsection{Two-level molecule}

The two-level molecule is described as the system of the ground state $|g\rangle$ and electronically excited state $|e\rangle$ as for the two-level atom. However, this system is additionally coupled to vibrational degrees of freedom. This coupling to these is expressed through the generalized bath coordinates (nuclear degrees of freedom) $\mathbf{Q}$. These coordinates introduce additional degrees of freedom (e. g. solvent or lattice kinetics, etc.). In the adiabatic regime the material Hamiltonian is

$$
\hat{H}_{\text {mat }}=|g\rangle H_{g}\langle g|+| e\rangle H_{e}\langle e| .
$$

Here Born-Oppenheimer approximation was used as well as ansatz of the absence of pathway of excited state relaxation to $|g\rangle$. On the other hand, full energy of the state is a sum of the electronically excited state level en$\operatorname{ergy} \varepsilon$, the kinetic energy of nuclear vibrations $T(\mathbf{Q})$, and the vibrational potential energy (adiabatic potential) $V(\mathbf{Q})$ (Fig. 5)

$$
\begin{aligned}
\hat{H}_{\mathrm{mat}} & =\left[\varepsilon_{g}+T(\mathbf{Q})+V_{g}(\mathbf{Q})\right]|g\rangle\langle g| \\
& +\left[\varepsilon_{e}+T(\mathbf{Q})+V_{e}(\mathbf{Q})\right]|e\rangle\langle e| .
\end{aligned}
$$

The Hamiltonian can be written in another form by separating the electronic and bath parts:

$$
\hat{H}_{\mathrm{mat}}=\hat{H}_{\mathrm{B}}+\hat{H}_{\mathrm{S}}+\hat{H}_{\mathrm{SB}} .
$$

Here $\hat{H}_{\mathrm{B}}$ is a purely vibrational part that depends solely on bath degrees of freedom, $\hat{H}_{\mathrm{S}}$ is a purely electronic part (system), and $\hat{H}_{\mathrm{SB}}$ is the electron-phonon coupling of system-bath interaction as defined in Sec. 2.4.1. These parts of molecular Hamiltonian can be written as

$$
\begin{aligned}
\hat{H}_{\mathrm{B}} & =\left[T(\mathbf{Q})+V_{g}(\mathbf{Q})\right](|g\rangle\langle g|+| e\rangle\langle e|), \\
\hat{H}_{\mathrm{S}} & =\varepsilon_{g}|g\rangle\left\langle g\left|+\left[\varepsilon_{e}+\left\langle V_{e}-V_{g}\right\rangle\right]\right| e\right\rangle\langle e|, \\
\hat{H}_{\mathrm{SB}} & =\left[V_{e}(\mathbf{Q})-V_{g}(\mathbf{Q})-\left\langle V_{e}-V_{g}\right\rangle\right]|e\rangle\langle e| .
\end{aligned}
$$

The average energy gap term $\left\langle V_{e}-V_{g}\right\rangle$ was added to the electronic part and subtracted from the interactional part (making zero contribution in the material Hamiltonian) assuming that the interaction part must be zero in the thermodynamical equilibrium. Thus the interaction part is an operator of energy gap fluctuations with respect to the ground state. The molecular Hamiltonian in the matrix notation for a two-level system then is very simple:

$$
\hat{H}_{\mathrm{mat}}=H_{\mathrm{B}}\left(\begin{array}{ll}
1 & 0 \\
0 & 1
\end{array}\right)+\left(\begin{array}{cc}
\varepsilon_{g} & 0 \\
0 & \bar{\varepsilon}_{e}
\end{array}\right)+\Delta V\left(\begin{array}{ll}
0 & 0 \\
0 & 1
\end{array}\right) .
$$

The dipole moment operator of a two-level system is written assuming Franck-Condon approximation, i.e. the polarization operator in the dipole limit is equivalent to the dipole operator, which does not depend on the vibrational coordinates:

$$
\hat{\mu}=d_{e g}|e\rangle\left\langle g\left|+d_{e g}^{*}\right| g\right\rangle\langle e| .
$$

The energy of the electronic transition is usually in UV or visible region and energy gap is greater than $k_{\mathrm{B}} T$, therefore we can assume that initially (at time $t \rightarrow-\infty)$ the system is in the equilibrium state, defined by equilibrium ground state density operator. So 
the dipole moment operator acting upon the equilibrium density matrix is

$$
\hat{\mu} \rho^{(\mathrm{eq})}=d_{e g} \rho^{(\mathrm{eq})}|e\rangle\langle g| .
$$

Linear response. Linear response function of the system is given by Eq. (67). In the following we include vibrational degrees of freedom. Now the response function depends on coordinates $\mathbf{Q}$.

$$
\begin{aligned}
J(t) & =\operatorname{Tr}_{\mathrm{B}}\left\{U^{\dagger}(t) \hat{\mu} U(t) \hat{\mu} \rho^{(\mathrm{eq})}\right\} \\
& =\left|d_{e g}\right|^{2} \operatorname{Tr}_{\mathrm{B}}\left\{\mathrm{e}^{\frac{\mathrm{i}}{\hbar} H_{g} t} \mathrm{e}^{-\frac{\mathrm{i}}{\hbar} H_{e} t} \rho^{(\mathrm{eq})}\right\} \\
& =\left|d_{e g}\right|^{2} \mathrm{e}^{-\mathrm{i} \omega_{e g} t} \operatorname{Tr}_{\mathrm{B}}\left\{\mathrm{e}^{-\frac{\mathrm{i}}{\hbar} \Delta V(\mathbf{Q}, t) t} \rho^{(\mathrm{eq})}\right\} .
\end{aligned}
$$

Here we extracted the energy gap $\omega_{e g}$ and in the exponential of the evolution operators only the vibrational part is left. Within the trace operation in the exponential we have a perturbative part of the excited state Hamiltonian, therefore, it is the excited state evolution operator in the interaction picture. It is known from the timedependent perturbation theory that it can be written as a positive time ordered exponential [29]

$$
\tilde{U}_{e}^{\mathrm{I}}(\mathbf{Q}, t)=\exp _{+}\left[-\frac{\mathrm{i}}{\hbar} \int_{0}^{t} \mathrm{~d} \tau \Delta V(\mathbf{Q}, \tau)\right] .
$$

By inserting this into $J(t)$ expression (Eq. (83)) we obtain

$$
\begin{aligned}
& J(t)=|d|^{2} \mathrm{e}^{-\mathrm{i} \omega_{e g} t} \\
& \times \operatorname{Tr}_{\mathrm{B}}\left\{\exp _{+}\left[-\frac{\mathrm{i}}{\hbar} \int_{0}^{t} \mathrm{~d} \tau \Delta V(\mathbf{Q}, \tau)\right] \rho_{\text {eq }}\right\} .
\end{aligned}
$$

Expanding Eq. (85) to the second order,

$$
\begin{aligned}
& J(t)=|d|^{2} \mathrm{e}^{\mathrm{i} \omega_{e g} t}\left\{1-\frac{\mathrm{i}}{\hbar} \int_{0}^{t} \mathrm{~d} \tau \operatorname{Tr}_{\mathrm{B}}\{\Delta V(\mathbf{Q}, \tau)\}(86)\right. \\
& +\left(\frac{\mathrm{i}}{\hbar}\right)^{2} \int_{0}^{t} \mathrm{~d} \tau \int_{0}^{\tau} \mathrm{d} \tau^{\prime} \operatorname{Tr}_{\mathrm{B}}\left\{\Delta V(\mathbf{Q}, \tau) \Delta V\left(\mathbf{Q}, \tau^{\prime}\right)\right\},
\end{aligned}
$$

we find that the second term in Eq. (86) is zero in the thermodynamical equilibrium. After performing a cumulant expansion (see Appendix (7) for details) of this expression, we obtain

$$
J(t)=|d|^{2} \mathrm{e}^{\mathrm{i} \omega_{e g} t-g(t)},
$$

where

$$
\begin{aligned}
g(t) \equiv & \left(\frac{1}{\hbar}\right)^{2} \int_{0}^{t} \mathrm{~d} \tau \int_{0}^{\tau} \mathrm{d} \tau^{\prime} \\
& \times \operatorname{Tr}_{\mathrm{B}}\left\{\Delta V(\mathbf{Q}, \tau) \Delta V\left(\mathbf{Q}, \tau^{\prime}\right) \rho_{\mathrm{eq}}\right\} .
\end{aligned}
$$

The function $g(t)$ defined here is called the lineshape function. It is a double integral of the energy gap correlation function:

$$
\begin{aligned}
C\left(\tau^{\prime}\right) & =\frac{1}{\hbar^{2}} \operatorname{Tr}_{\mathrm{B}}\left\{\Delta V\left(\mathbf{Q}, \tau^{\prime}\right) \Delta V(\mathbf{Q}, 0) \rho_{\mathrm{eq}}\right\}, \\
g(t) & =\int_{0}^{t} \mathrm{~d} \tau \int_{0}^{\tau} \mathrm{d} \tau^{\prime} C\left(\tau^{\prime}\right) .
\end{aligned}
$$

Having the linear response function derived we are now able to obtain full expression of the absorption spectra of the two-level system. To make it even more simpler, a fast modulation limit approximation will be used. It is based on the assumption that the bath fluctuations are much more intensive than those of the system vibrations. Then the system-bath coupling is "fast" and the energy gap correlation function is assumed to be of shape of the Dirac delta function:

$$
C(t) \equiv \gamma \delta(t) .
$$

Here $\gamma$ is the coupling strength. This type of correlation is denoted as Markovian since the memory of system initial state is lost extremely fast. That is the case used for calculating expressions for the two-level atom in Section 3.1.

We can, in advance, notice that in this regime the Stokes shift will be absent since due to the ultra-fast bath kinetics the electric field always interacts with an averaged ensemble of two-level systems [29]. By applying a double integration of the $\delta$-shaped correlation function, we get a linear expression of the $g(t)$ function:

$$
g(t)=\gamma t .
$$

An analytic expression of the absorption coefficient is then obtained by applying Fourier transform to the linear response function

$$
\begin{aligned}
\kappa_{\alpha}(\omega) & \propto \omega|d|^{2} \operatorname{Re} \int_{0}^{\infty} \mathrm{d} t \mathrm{e}^{\mathrm{i}\left(\omega-\omega_{e g}\right) t-\Gamma t} \\
& =|d|^{2} \omega \operatorname{Re} \frac{1}{\omega-\omega_{e g}+\mathrm{i} \gamma} .
\end{aligned}
$$


The resulting lineshape is Lorentzian (Fig. 5) centreed at $\omega_{e g}$ with $2 \gamma$ for the Full Width at Half Maximum (FWHM) as a signature of homogeneous broadening in the absorption spectrum. Therefore the fast modulation limit is also known as the homogeneous limit.

In contrast to the homogeneous limit, the inhomogeneous limit can also be described. If the system does not lose memory of its preceding states, the correlation function is constant, $C(t) \equiv C_{0}$. The resulting lineshape function is $g(t)=\frac{1}{2} C_{0} t^{2}$. The spectral lineshape is Gaussian with $2 \sqrt{2 \ln 2 C_{0}^{-1}}$ for the FWHM.

Third-order response and 2D spectrum. Spectra of inhomogeneous system are shown in Fig. 6. For the real part of the rephasing spectrum the diagonal peak lineshape is Gaussian (c. f. Lorentzian lineshapes of Fig. 4) and elongated with respect to the main diagonal. It is an illustration of the fact that the inhomogeneously broadened spectrum can be assumed as the superposition of spectra of two-level systems with different toplevel energies that are Gaussian-distributed. If the inhomogeneity is included, a decay of non-rephasing signal is also evident. This is caused by destructive addition of positive and negative diagonal values of the signal. Thus the rephasing signal gives more information about the system since the homogeneous and inhomogeneous contributions are well separated.

The angle of nodal line (separating positive and negative off-diagonal peaks) of the imaginary part of the sum spectrum also characterizes the system under consideration. In this case it is the ratio of inhomogeneous and homogeneous contributions. This type of counter-clockwise nodal line rotation is observed when the energy-gap correlation function is of type of slow exponential decay (static inhomogeneous broadening) [21] or as a result of losing of memory of the initial excitation of the system [18].

\subsection{Anharmonic oscillator (three-level system)}

Here we describe the linear and third-order response of a weakly anharmonic oscillator. In this case the molecular Hamiltonian is

$$
\hat{H}_{\mathrm{mol}}=\omega_{0} \hat{a}^{\dagger} \hat{a}+\frac{\Delta}{2} \hat{a}^{\dagger} \hat{a}^{\dagger} \hat{a} \hat{a},
$$

where $\hat{a}$ and $\hat{a}^{\dagger}$ are bosonic annihilation and creation operators, $\omega_{0}$ is known as the fundamental frequency, and $\Delta \ll \omega_{0}$ is the anharmonicity. Three lowest states of this system are the ground state $|g\rangle$ with energy 0 , the one-quantum state $|e\rangle$ with the energy $\omega_{0}$, and the

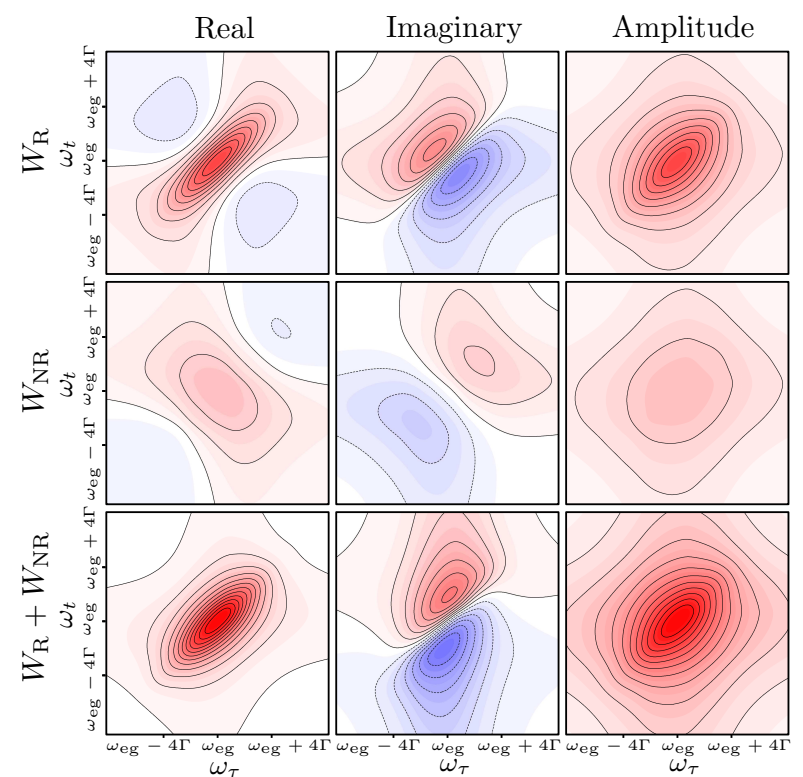

Fig. 6. Calculated rephasing, non-rephasing, and sum spectra for the real (absorptive signal) and imaginary (dispersive signal) parts and signal amplitude of a single two-level system at fast modulation limit with Gaussian disorder of $4 \gamma$ for the FWHM.

double-quantum state $|f\rangle$ with energy $2 \omega_{0}+\Delta$. The dipole operator is

$$
\hat{H}_{\mathrm{SF}}=\mu\left(\hat{a}^{\dagger}+\hat{a}\right) .
$$

This gives the fundamental transition amplitude $\mu_{g e}=$ $\mu$ and the transition from the one-quantum state to the double-quantum state equal to $\mu_{e f}=\sqrt{2} \mu$.

The connection with the oscillator can be easily established by introducing the dimensionless coordinate $\hat{q}$ and momentum $\hat{p}$ :

$$
\begin{aligned}
& \hat{q}=\frac{1}{\sqrt{2}}\left(\hat{a}^{\dagger}+\hat{a}\right), \\
& \hat{p}=\frac{\mathrm{i}}{\sqrt{2}}\left(\hat{a}^{\dagger}-\hat{a}\right) .
\end{aligned}
$$

The linear response function has the same form as the two-level system since the overtone state is not involved (Eq. (67)).

In the photon echo rephasing response function we now have three contributions. Due to the presence of 
the double-quantum state $|f\rangle$, the negative induced absorption contribution adds up,

$$
\begin{aligned}
S^{\mathrm{IA}}\left(t_{3}, t_{2}, t_{1}\right) & =R_{1}\left(t_{3}, t_{2}, t_{1}\right) \\
& =-2 \mu^{4} \mathrm{e}^{\mathrm{i} \omega_{0}\left(t_{1}-t_{3}\right)-\mathrm{i} \Delta t_{3}-\gamma\left(t_{1}+t_{3}\right)} \\
S^{\mathrm{GSB}}\left(t_{3}, t_{2}, t_{1}\right) & =R_{2}\left(t_{3}, t_{2}, t_{1}\right) \\
& =\mu^{4} \mathrm{e}^{\mathrm{i} \omega_{0}\left(t_{1}-t_{3}\right)-\gamma\left(t_{1}+t_{3}\right)} \\
S^{\mathrm{SE}}\left(t_{3}, t_{2}, t_{1}\right) & =R_{3}\left(t_{3}, t_{2}, t_{1}\right) \\
& =\mu^{4} \mathrm{e}^{\mathrm{i} \omega_{0}\left(t_{1}-t_{3}\right)-\gamma\left(t_{1}+t_{3}\right)}
\end{aligned}
$$

After Fourier transformations we have

$$
\begin{aligned}
& W_{\mathrm{R}}\left(\omega_{\tau}, T, \omega_{t}\right)=2\left(\frac{i}{\hbar}\right)^{3} \theta(T) \frac{\mu^{4}}{\gamma-\mathrm{i}\left(\omega_{\tau}-\omega_{0}\right)} \\
& \times\left[\frac{1}{\gamma+\mathrm{i}\left(\omega_{t}-\omega_{0}\right)}-\frac{1}{\gamma+\mathrm{i}\left(\omega_{t}-\omega_{0}-\Delta\right)}\right] .
\end{aligned}
$$

Similarly, for the non-rephasing contribution we would obtain

$$
\begin{aligned}
& W_{\mathrm{NR}}\left(\omega_{\tau}, T, \omega_{t}\right)=2\left(\frac{i}{\hbar}\right)^{3} \theta(T) \frac{\mu^{4}}{\gamma-\mathrm{i}\left(\omega_{\tau}-\omega_{0}\right)} \\
& \times\left[\frac{1}{\gamma-\mathrm{i}\left(\omega_{t}-\omega_{0}\right)}-\frac{1}{\gamma-\mathrm{i}\left(\omega_{t}-\omega_{0}-\Delta\right)}\right] .
\end{aligned}
$$

The total spectrum

$$
\begin{aligned}
& W\left(\omega_{\tau}, T, \omega_{t}\right)=4\left(\frac{\mathrm{i}}{\hbar}\right)^{3} \theta(T) \frac{\gamma^{2}+\mathrm{i} \gamma\left(\omega_{\tau}-\omega_{0}\right)}{\gamma^{2}+\left(\omega_{\tau}-\omega_{0}\right)^{2}} \\
& \times\left[\frac{1}{\gamma^{2}+\left(\omega_{t}-\omega_{0}\right)^{2}}-\frac{1}{\gamma^{2}+\left(\omega_{t}-\omega_{0}-\Delta\right)^{2}}\right] .
\end{aligned}
$$

It is seen that in case of no anharmonicity $(\Delta=0)$, IA contribution cancels SE and GSB pathways completely. If anharmonicity is large compared to the dephasing constant $(\Delta>\gamma)$, negative and positive peaks are well resolved in 2D spectrum (Fig. 7). In the other case, positive and negative peaks overlap and the nodal line can be drawn.

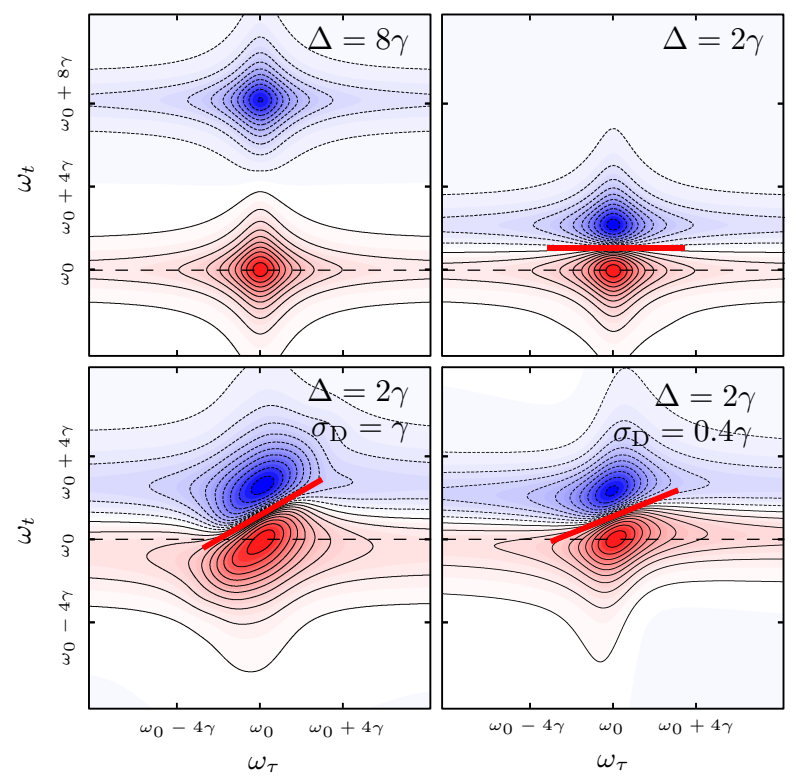

Fig. 7. Real part of the total $W\left(\omega_{\tau}, T, \omega_{t}\right)$ two-dimensional spectra of a three-level system (anharmonic oscillator). $\Delta$ denotes an anharmonicity, $\sigma_{\mathrm{D}}$ is the standard deviation of the Gaussiandistributed diagonal disorder, $\gamma$ is pure dephasing rate.

\subsection{Multi-level system in contact with the bath: cumulant expansion of the third-order response function}

We assume that the system is given by a set of energy states: the ground state $|g\rangle$ and $|1\rangle,|2\rangle \ldots|N\rangle$ states. The molecular Hamiltonian is thus

$$
\hat{H}_{\mathrm{mol}}=\sum_{a=1}^{N} \varepsilon_{a}|a\rangle\langle a| .
$$

Here and later roman symbols $a, b$, and $c$ run over all the energy states in summations. Again the bath is described by the set $\{\alpha\}$ of harmonic oscillators. The system-bath interaction is given by

$\hat{H}_{\mathrm{SB}}=\sqrt{2} \sum_{a b \alpha} d_{a b}^{(\alpha)} Q_{\alpha}|a\rangle\left\langle b\left|\equiv \sum_{a b \alpha} d_{a b}^{(\alpha)}\left(\hat{b}_{\alpha}^{\dagger}+\hat{b}_{\alpha}\right)\right| a\right\rangle\langle b|$.

We first neglect off-diagonal fluctuations. In that case the system dynamics is adiabatic, i.e. the system eigenstates are not affected by the fluctuations. System response functions $R_{1}\left(t_{3}, t_{2}, t_{1}\right), \ldots R_{4}\left(t_{3}, t_{2}, t_{1}\right)$ (Eqs. (46)-(49) ) can then be written as four-point 
correlation functions of the system and dipole moment operator interaction times

$$
\begin{aligned}
& R_{1}\left(t_{3}, t_{2}, t_{1}\right)=F\left(t_{1}, t_{1}+t_{2}, t_{1}+t_{2}+t_{3}, 0\right), \\
& R_{2}\left(t_{3}, t_{2}, t_{1}\right)=F\left(0, t_{1}+t_{2}, t_{1}+t_{2}+t_{3}, t_{1}\right), \\
& R_{3}\left(t_{3}, t_{2}, t_{1}\right)=F\left(0, t_{1}, t_{1}+t_{2}+t_{3}, t_{1}+t_{2}\right), \\
& R_{4}\left(t_{3}, t_{2}, t_{1}\right)=F\left(t_{1}+t_{2}+t_{3}, t_{1}+t_{2}, t_{1}, 0\right),
\end{aligned}
$$

where

$$
F\left(\tau_{4}, \tau_{3}, \tau_{2}, \tau_{1}\right)=\operatorname{Tr}_{\mathrm{B}}\left\{\hat{\mu}\left(\tau_{4}\right) \hat{\mu}\left(\tau_{3}\right) \hat{\mu}\left(\tau_{2}\right) \hat{\mu}\left(\tau_{1}\right) \rho_{\mathrm{eq}}\right\}
$$

and $\hat{\mu}(\tau)=\mathscr{U}(\tau) \hat{\mu} ; \hat{\mu}$ is defined in Eq. (130). The evolution superoperator (as in Sec. 3.2) can be expressed as a product of a normal and time-ordered exponential (representing system and bath fluctuations, respectively)

$$
\mathscr{U}_{a b}(\tau)=\exp \left(-\mathrm{i} \omega_{a b} \tau\right) \exp _{+}\left[-\mathrm{i} \int_{0}^{\tau} \mathrm{d} \tau^{\prime} \Delta V_{a b}(\tau)\right]
$$

Applying the four-point correlation function to a general scheme of system-dipole moment operator interactions (Fig. 3), one can obtain

$$
\begin{aligned}
& F\left(\tau_{4}, \tau_{3}, \tau_{2}, \tau_{1}\right) \\
& =\sum_{c b a} \mu_{g c} \mu_{c b} \mu_{b a} \mu_{a g} \mathscr{F}_{c b a}^{(\mathrm{C})}\left(\tau_{4}, \tau_{3}, \tau_{2}, \tau_{1}\right) .
\end{aligned}
$$

Index $\mathrm{C}$ denotes the coherent limit - population transfer is not included. The four-point correlation function is then

$$
\begin{aligned}
& \mathscr{F}_{c b a}^{(\mathrm{C})}\left(\tau_{4}, \tau_{3}, \tau_{2}, \tau_{1}\right)=\exp \left[-\mathrm{i}\left(\varepsilon_{c} \tau_{43}+\varepsilon_{b} \tau_{32}+\varepsilon_{a} \tau_{21}\right)\right. \\
& \left.+f_{c b a}^{(\mathrm{C})}\left(\tau_{4}, \tau_{3}, \tau_{2}, \tau_{1}\right)\right],
\end{aligned}
$$

where

$$
\begin{aligned}
& f_{c b a}^{(\mathrm{C})}\left(\tau_{4}, \tau_{3}, \tau_{2}, \tau_{1}\right) \\
&=\operatorname{Tr}_{\mathrm{B}}\left\{\mathrm{e}_{+}^{-\mathrm{i} \int_{0}^{\tau_{1}} \mathrm{~d} \tau \Delta V_{g c}(\tau)} \mathrm{e}_{+}^{-\mathrm{i} \int_{0}^{\tau_{2}} \mathrm{~d} \tau \Delta V_{c b}(\tau)}\right. \\
&\left.\quad \mathrm{e}_{+}^{-\mathrm{i} \int_{0}^{\tau_{3}} \mathrm{~d} \tau \Delta V_{b a}(\tau)} \mathrm{e}_{+}^{-\mathrm{i} \int_{0}^{\tau_{4}} \mathrm{~d} \tau \Delta V_{a g}(\tau)}\right\}
\end{aligned}
$$

The second-order cumulant expansion of this expression (for details see Appendix 7) results in

$$
\begin{aligned}
& f_{c b a}^{(\mathrm{C})}\left(\tau_{4}, \tau_{3}, \tau_{2}, \tau_{1}\right) \\
& =\exp \left[-g_{c c}\left(\tau_{43}\right)-g_{b b}\left(\tau_{32}\right)-g_{a a}\left(\tau_{21}\right)\right. \\
& -g_{c b}\left(\tau_{42}\right)+g_{c b}\left(\tau_{43}\right)+g_{c b}\left(\tau_{32}\right) \\
& -g_{c a}\left(\tau_{41}\right)+g_{c a}\left(\tau_{42}\right)+g_{c a}\left(\tau_{31}\right)-g_{c a}\left(\tau_{32}\right) \\
& \left.-g_{b a}\left(\tau_{31}\right)+g_{b a}\left(\tau_{32}\right)+g_{b a}\left(\tau_{21}\right)\right] .
\end{aligned}
$$

The lineshape function $g_{a b}(t)$ is given by the correlation function $C_{a a, b b}(t)$ integral [29]:

$$
g_{a b}(t)=\int_{0}^{t} \mathrm{~d} \tau \int_{0}^{\tau} \mathrm{d} \tau^{\prime} C_{a a, b b}\left(\tau-\tau^{\prime}\right) .
$$

Consider now additional off-diagonal fluctuations. Now the system dynamics includes population transport and the system cannot be described using evolution operators of the wave function. Using the secular approximation for the system dynamics it can separated into two types: the coherence evolution and the population transport. During the coherence evolution, the diagonal fluctuations modulate the oscillation frequency, off-diagonal fluctuations, and the lifetimeinduced dephasing. During the population evolution, the off-diagonal fluctuations induce population transport and the effect of the diagonal fluctuations is included in the transport rate. For Feynman diagrams, where population transfer is involved, diagrams with incoherent transport are used [32]. The addition to the system response function (Eq. (44)) is

$$
\begin{aligned}
& T\left(t_{3}, t_{2}, t_{1}\right)=-(\mathrm{i})^{3} \sum_{c b e^{\prime} e} \mu_{c b} \mu_{\nu \nu^{\prime}} \mu_{e g}^{2} G_{e^{\prime} e}\left(t_{2}\right) \\
& \times \mathscr{F}_{c b e^{\prime} e}^{(\mathrm{I})}\left(t_{3}, t_{2}, t_{1}\right),
\end{aligned}
$$

where indices $c$ and $b$ denote states in coherence $|b\rangle\langle c|$ during $t_{3}$ in population transfer diagrams; $\nu \nu^{\prime}$ has to be changed to $e^{\prime} b$ when the signal is generated on the left side of the diagram, and to $c e^{\prime}$ when it is generated on the right. $G_{e^{\prime} e}\left(t_{2}\right)$ is Green's function that is a probability of population state $|e\rangle\langle e|$ to be transferred to $\left|e^{\prime}\right\rangle\left\langle e^{\prime}\right|$ in time $t_{2}$ (or population survival probability when $e^{\prime}=e$ ).

$$
\begin{aligned}
\mathscr{F}_{c b e^{\prime} e}^{(\mathrm{I})}\left(t_{3}, t_{2}, t_{1}\right) & =\exp \left[\mathrm{i} \omega_{c b} t_{3}-\mathrm{i} \omega_{e g} t_{1}-\left(\gamma_{c}+\gamma_{b}\right) t_{3}\right. \\
-\gamma_{e} t_{1} & \left.+f_{c b e}^{(\mathrm{I})}\left(t_{3}, t_{2}, t_{1}\right)\right],
\end{aligned}
$$


where

$$
\begin{aligned}
& f_{c b e}^{(\mathrm{I})}\left(t_{3}, t_{2}, t_{1}\right)=\exp \left[-g_{e e}\left(t_{1}\right)-g_{b b}\left(t_{3}\right)-g_{c c}^{*}\left(t_{3}\right)\right. \\
& -g_{b e}\left(t_{1}+t_{2}+t_{3}\right)+g_{b e}\left(t_{1}+t_{2}\right)+g_{b e}\left(t_{2}+t_{3}\right) \\
& +g_{c e}\left(t_{1}+t_{2}+t_{3}\right)-g_{c e}\left(t_{1}+t_{2}\right)-g_{c e}\left(t_{2}+t_{3}\right) \\
& \left.+g_{c b}\left(t_{3}\right)+g_{b c}^{*}\left(t_{3}\right)+g_{c e}\left(t_{2}\right)-g_{b e}\left(t_{2}\right)\right] .
\end{aligned}
$$

$\gamma_{\nu}$ is the dephasing constant, that is a sum of a state lifetime and pure dephasing

$$
\gamma_{\nu}=\left|K_{\nu \nu}\right| / 2+\tilde{\gamma}_{\nu}
$$

The population Green's function is a solution of the Pauli master equation

$$
\dot{G}_{e^{\prime} e}(t)=\sum_{j \neq e^{\prime}} K_{e^{\prime} j} G_{j e}-\left(\sum_{j \neq e^{\prime}} K_{j e^{\prime}}\right) G_{e^{\prime} e},
$$

where $K_{i j}$ are the population transport rates. This equation can be represented in a matrix form

$$
\frac{\partial}{\partial t} \hat{G}(t)=-\hat{\tilde{K}} \hat{G}(t),
$$

where the population transport rate matrix is constructed as $\tilde{K}_{a b}=-K_{a b}+\delta_{a b} \sum_{j} K_{j b}$. By applying unitary transformation upon the explicit solution of Eq. (116)) we obtain

$$
\hat{G}(t)=\hat{Q} \exp \left(-\hat{Q}^{-1} \hat{\tilde{K}} \hat{Q} t\right) \hat{Q}^{-1}
$$

and

$$
G_{e^{\prime} e}(t)=\sum_{j} Q_{e^{\prime} j} Q_{j e}^{-1} \mathrm{e}^{-\lambda_{j} t}
$$

Here $\hat{Q}$ is eigenvector matrix of $\hat{\tilde{K}}, \hat{Q}^{-1}$ is its inverse, and $\lambda_{j}$ are the eigenvalues. Population transport rates can be calculated using traditional Redfield theory [27, 33]:

$$
K_{a b}=\operatorname{Re} C_{a b, a b}^{\prime \prime}\left(\omega_{a b}\right)\left[\operatorname{coth}\left(\beta \hbar \omega_{a b} / 2\right)-1\right],
$$

where $\omega_{a b}=\varepsilon_{a}-\varepsilon_{b}, \beta=\left(k_{\mathrm{B}} T\right)^{-1}, T$ is the temperature, $k_{\mathrm{B}}$ is Boltzmann constant, and

$$
C_{a b, c d}^{\prime \prime}(\omega)=\frac{1-\exp (-\beta \hbar \omega)}{2} \int_{-\infty}^{\infty} \mathrm{d} t \mathrm{e}^{\mathrm{i} \omega t} C_{a b, c d}(t) .
$$

\section{Coupled multi-chromophore system}

\subsection{Frenkel exciton model}

We consider a general multi-chromophore system consisting of $N$ electronically interacting two-level subsystems. Nonlinear optical properties of such complexes of coupled chromophores (e.g. molecular aggregates, proteins etc.) are described using a Frenkel exciton model [34, 35]. The Frenkel exciton Hamiltonian is

$$
\hat{H}=\sum_{m=1}^{N} \epsilon_{m}|m\rangle\left\langle m\left|+\sum_{m}^{N} \sum_{n \neq m}^{N} J_{n m}\right| n\right\rangle\langle m|+\hat{H}_{\text {int }},
$$

where $\epsilon_{m}$ and $J_{m n}$ are site energy of the $m$ th chromophore and the resonant coupling between $n$th and $m$ th chromophores, respectively. $\hat{H}_{\text {int }}$ denotes interaction to the field and the environment and is treated as a weak perturbation. In the exciton representation a multi-level system is considered. It contains the ground state $|g\rangle$ and one- and two-exciton bands (manifolds). Each state of the one-exciton manifold is denoted as $\left|e_{j}\right\rangle(j=1 \ldots N)$ with corresponding optical transition to the ground state $\omega_{e_{j} g}$. The number of twoexciton states is $N(N-1) / 2$ and they are denoted as $\left|f_{k}\right\rangle$ with optical transitions to the one-exciton band $\omega_{f_{k} e_{j}}$; transition to the ground state is forbidden. The one-exciton Hamiltonian matrix $\hat{h}^{(1)}$ is simply the reference Hamiltonian of Eq. (121): $h_{j k}^{(1)}=\delta_{j k} \epsilon_{j}+\zeta_{j k} J_{j k}$, where $\zeta_{j k}=1-\delta_{j k}$. Two-exciton Hamiltonian $h^{(2)}$ is $h_{(k l),(m n)}^{(2)} \equiv\left(\epsilon_{k}+\epsilon_{l}\right) \delta_{k m} \delta_{l n}+J_{k m} \delta_{l n} \zeta_{k m}+J_{l n} \delta_{k m} \zeta_{l n}$. Transition from site representation to the exciton basis as well as eigen-energies are obtained using unitary transformations

$$
\begin{aligned}
& U^{-1} \hat{h}^{(1)} U=\Omega, \\
& V^{-1} \hat{h}^{(2)} V=W .
\end{aligned}
$$

$\hat{H}_{\text {int }}=\hat{H}_{\mathrm{SF}}+\hat{H}_{\mathrm{SB}}$ determines the system interactions with external perturbations: the optical field (denoted as SF, "system-field") and the phonon bath (SB, "system-bath"). The former is given by

$$
\hat{H}_{\mathrm{SF}}=\sum_{m} \mathbf{d}_{m}(|m\rangle+\langle m|),
$$



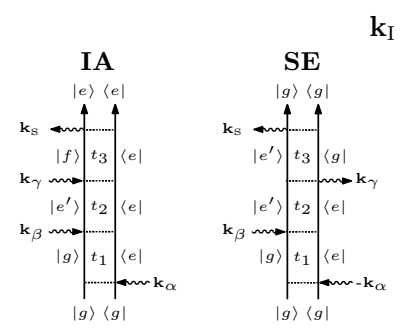

$\mathbf{k}_{\mathrm{I}}=-\mathbf{k}_{\alpha}+\mathbf{k}_{\beta}+\mathbf{k}_{\gamma}$
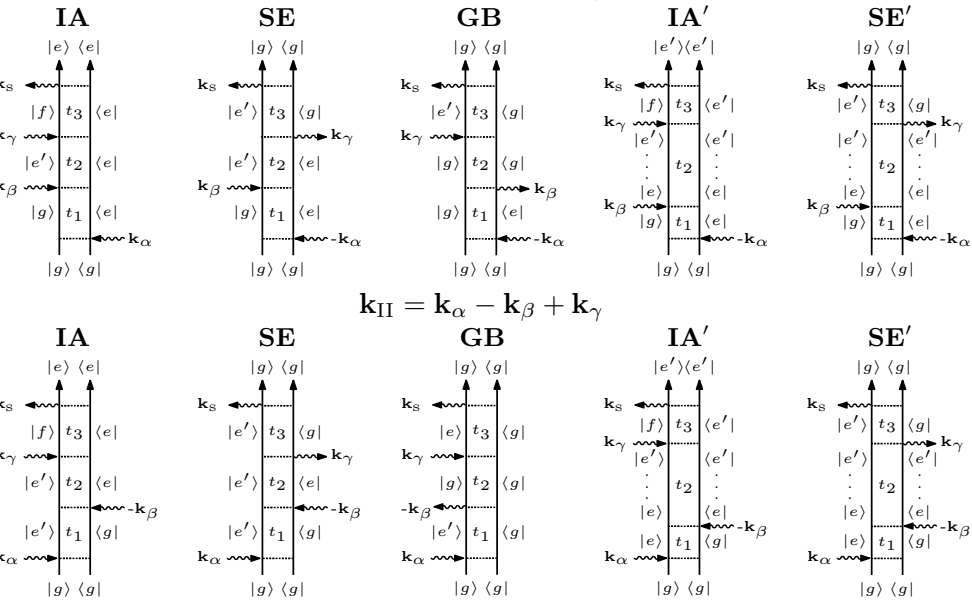

$\mathbf{k}_{\mathrm{II}}=\mathbf{k}_{\alpha}-\mathbf{k}_{\beta}+\mathbf{k}_{\gamma}$
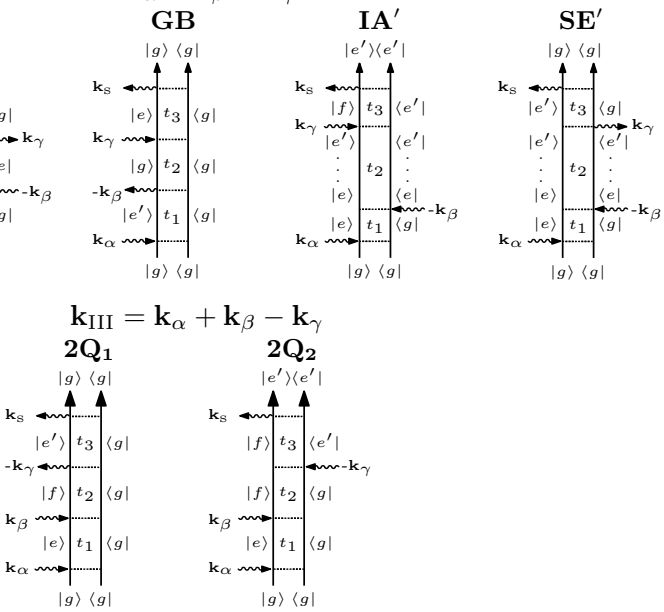

Fig. 8. Double-sided Feynman diagrams, illustrating Liouville space pathways (LSPs) in the system, induced by a repetitive dipole moment interaction with both sides of the system density operator.

where $\mathbf{d}$ is a molecular transition dipole. Applying the unitary transformation the dipoles of intra-band transitions (eigen-dipoles) are obtained:

$$
\begin{aligned}
\mu_{g e_{j}} & =\sum_{m} U_{j m}^{-1} \mathbf{d}_{m} \\
\mu_{e_{j} f_{k}} & =\sum_{m=1}^{N-1} \sum_{n=m+1}^{N} \nu_{m n}^{(k)}\left(U_{j n}^{-1} \mathbf{d}_{m}+U_{j m}^{-1} \mathbf{d}_{n}\right) .
\end{aligned}
$$

Here $\nu_{n m}^{(k)}$ denotes the element of $V^{-1}$ of the $k$ th row and the column, corresponding to a diagonal element $\epsilon_{n}+\epsilon_{m}$ of the two-exciton Hamiltonian matrix. The explicit relation is $\nu_{m n}^{(k)}=V_{k \gamma}^{-1}$, where $\gamma=\frac{1}{2}(2 N-$ $m)(m-1)+n-m, n, m=1,2 \ldots N$. We next assume that $\hat{H}_{\mathrm{SB}}$ term induces fluctuations of the molecular transition energies:

$$
\hat{H}_{\mathrm{SB}}=\sum_{m} q_{m}(\mathbf{Q}, t)|m\rangle\langle m|,
$$

where $\mathbf{Q}$ are the collective phonon bath coordinates. We assume that each molecule has its own independent set of fluctuating coordinates uncorrelated with the other molecules. Fluctuations of different molecules are statistically independent, i.e. the correlation function matrix is diagonal, $\left\langle q_{m}(t) q_{n}(0)\right\rangle=\delta_{m n} C(t)$. It is convenient to use the spectral density, which is given by [27]:

$$
\mathscr{C}^{\prime \prime}(\omega)=\frac{1}{2} \int_{0}^{\infty} \mathrm{d} t \exp (\mathrm{i} \omega t)\left\langle\left[q_{m}(t), q_{m}(0)\right]\right\rangle .
$$

In the exciton basis, we obtain fluctuating transition energies and couplings between the eigenstates. These fluctuations are characterized by spectral densities

$$
\begin{aligned}
& C_{e_{1} e_{2}, e_{3} e_{4}}^{\prime \prime}(\omega) \\
& =\left[\sum_{m} U_{m e_{1}}^{-1} U_{m e_{2}}^{-1} U_{m e_{3}}^{-1} U_{m e_{4}}^{-1}\right] \mathscr{C}^{\prime \prime}(\omega), \\
& C_{e_{1} e_{2}, f_{3} f_{4}}^{\prime \prime}(\omega) \\
& =\left[\sum_{m} U_{m e_{1}}^{-1} U_{m e_{2}}^{-1} \sum_{k}^{k \neq m} \nu_{m k}^{\left(f_{3}\right)} \nu_{m k}^{\left(f_{4}\right)}\right] \mathscr{C}^{\prime \prime}(\omega), \\
& C_{f_{1} f_{2}, e_{3} e_{4}}^{\prime \prime \prime}(\omega)=C_{e_{3} e_{4}, f_{1} f_{2}}(\omega) \\
& C_{f_{1} f_{2}, f_{3} f_{4}}^{\prime \prime \prime}(\omega) \\
& =\left[\sum_{m}\left(\sum_{k}^{k \neq m} \nu_{m k}^{\left(f_{1}\right)} \nu_{m k}^{\left(f_{2}\right)}\right)\left(\sum_{l}^{l \neq m} \nu_{m l}^{\left(f_{3}\right)} \nu_{m l}^{\left(f_{4}\right)}\right)\right] \mathscr{C}^{\prime \prime}(\omega) .
\end{aligned}
$$


Here we have extended the double-exciton eigenvector matrix by taking $\nu_{m n}^{(f)} \equiv \nu_{n m}^{(f)}$, which lets us simplify the expressions considerably.

\subsection{System response function of three-manifold system}

The dipole moment operator for an excitonic multilevel system described above is

$$
\hat{\mu}=\sum_{e} \mu_{e}|e\rangle\left\langle g\left|+\sum_{e, f}\right| f\right\rangle\langle e|+\text { h.c. }
$$

In this definition, dipole moments of transitions between excitonic states are not operators due to FranckCondon approximation (dipole moments are excitationindependent). The system also does not have a permanent dipole moment $(\langle g|\hat{\mu}| g\rangle=0)$. By inserting Eq. (130) into expressions of elements of the response function (Eqs. $(46)-(49)$ ) we obtain oscillating terms of the evolution operator in frequency $\bar{\omega}$ of transitions between excitonic manifolds and ground state (approx. the same frequency for all inter-band transitions). Separating the phase of resulting expressions one can see that there are two types of phase factors, $-\mathrm{i} \bar{\omega}\left(t_{1}+t_{3}\right)$ and $-\mathbf{i} \bar{\omega}\left(t_{1}-t_{3}\right)$. The opposite phase factors of electromagnetic field oscillations of frequency $\omega_{0}$ are in electromagnetic field expression Eq. (61). In the experiment $\omega_{0} \approx \bar{\omega}$ is set. Multiplication of the system response function and the electric field part under integration in Eq. (42) then results in terms of sum phase factors. In case of constructive (destructive) interference of phase factors rapidly (slowly) oscillating terms are obtained. Integrals of rapidly-varying functions are much smaller and can be neglected (RWA). The thirdorder polarization then is

$$
\begin{aligned}
& P^{(3)}(\tau, T, t)=\mathrm{e}^{-\mathrm{i} \omega_{0}(t-\tau)} \int_{0}^{\infty} \int_{0}^{\infty} \int_{0}^{\infty} \mathrm{d} t_{3} \mathrm{~d} t_{2} \mathrm{~d} t_{1} \\
& \times\left\{S_{\mathrm{I}}^{(3)} \times\left[\bar{E}_{1}^{[1]} E_{2}^{[2]} E_{3}^{[3]}+\bar{E}_{1}^{[1]} E_{3}^{[2]} E_{2}^{[3]}\right] \mathrm{e}^{\mathrm{i} \omega_{0}\left(t_{3}-t_{1}\right)}\right. \\
& +S_{\mathrm{II}}^{(3)} \times\left[E_{2}^{[1]} \bar{E}_{1}^{[2]} E_{3}^{[3]}+E_{3}^{[1]} \bar{E}_{1}^{[2]} E_{2}^{[3]}\right] \mathrm{e}^{\mathrm{i} \omega_{0}\left(t_{3}+t_{1}\right)} \\
& \left.+S_{\mathrm{III}}^{(3)} \times\left[E_{2}^{[1]} E_{3}^{[2]} \bar{E}_{1}^{[3]}+E_{3}^{[1]} E_{2}^{[2]} \bar{E}_{1}^{[3]}\right] \mathrm{e}^{\mathrm{i} \omega_{0}\left(t_{3}+2 t_{2}+t_{1}\right)}\right\},
\end{aligned}
$$

where $S_{\mathrm{I}}^{(3)}=S_{\mathrm{I}}^{(3)}\left(t_{3}, t_{2}, t_{1}\right)=-R_{1}^{*}+R_{2}+R_{3}$, $S_{\mathrm{II}}^{(3)}=S_{\mathrm{II}}^{(3)}\left(t_{3}, t_{2}, t_{1}\right)=-R_{2}^{*}+R_{1}+R_{4}$, and $S_{\mathrm{III}}^{(3)}=$ $S_{\mathrm{III}}^{(3)}\left(t_{3}, t_{2}, t_{1}\right)=-R_{3}^{*}+R_{4}$ are the system response functions for $\mathbf{k}_{\mathrm{I}}, \mathbf{k}_{\mathrm{II}}$, and $\mathbf{k}_{\mathrm{III}}$ interaction sequences, respectively. These interaction sequences of the evolu-
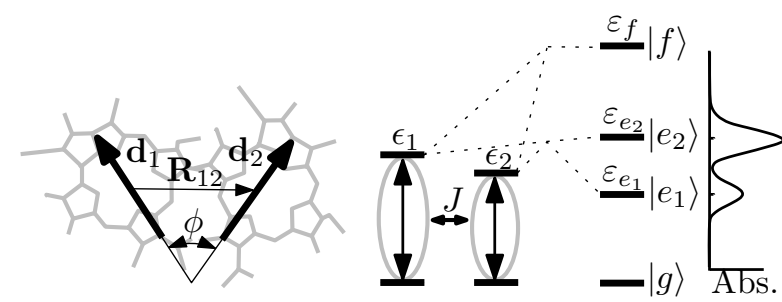

Fig. 9. Site representation and formation of molecular excitations (molecular excitons) of a general hetero-dimer system and illustration of absorption spectrum with peaks corresponding to optical transitions from the ground state to single-exciton states.

tion operator and the dipole moment operator can be expressed by double-sided Feynman diagrams (Fig. 8). They correspond to different physical processes - induced absorption ( $-R_{1}^{*}$ and $-R_{2}^{*}$ ), stimulated emission ( $R_{2}$ and $R_{1}$ ), ground state bleaching $\left(R_{3}\right.$ and $\left.R_{4}\right)$, or double-coherence pathways $\left(-R_{3}^{*}\right.$ and $\left.R_{4}\right)$. In further discussion we will use notation of these diagrams instead of $R$ functions. Complete expressions for $S_{\mathrm{I}}$ and respective diagrams are presented in Appendix 7 .

\section{Spectroscopy of dimers}

\subsection{Dimer of two-level chromophores}

An excitonically coupled dimer is an archetypical molecular system to be analysed by methods of optical 2D spectroscopy. The general scheme of a heterodimer as well as the exciton band structure are presented in Fig. 9. The dimer consists of two coupled chromophores, represented by two dipoles $\mathbf{d}_{1}$ and $\mathbf{d}_{2}$ with interdipole distance vector $\mathbf{R}_{12}$ and angle $\phi$. In the Frenkel exciton Hamiltonian the chromophore energies are denoted as $\epsilon_{1}$ and $\epsilon_{2}$ and the the coupling constant $J$,

$$
\hat{H}_{\mathrm{mol}}=\epsilon_{1} \hat{a}_{1}^{\dagger} \hat{a}_{1}+\epsilon_{2} \hat{a}_{2}^{\dagger} \hat{a}_{2}+J\left(\hat{a}_{1}^{\dagger} \hat{a}_{2}+\hat{a}_{2}^{\dagger} \hat{a}_{1}\right) .
$$

The Hamiltonian is diagonalized and the eigenenergies for the single-exciton states are

$$
\varepsilon_{e_{1}}=\epsilon_{1} \cos ^{2} \theta+\epsilon_{2} \sin ^{2} \theta-2 J \cos \theta \sin \theta,
$$

$$
\varepsilon_{e_{2}}=\epsilon_{1} \sin ^{2} \theta+\epsilon_{2} \cos ^{2} \theta+2 J \cos \theta \sin \theta,
$$

and

$$
\varepsilon_{f}=\varepsilon_{e_{1}}+\varepsilon_{e_{2}}
$$


for the double-exciton state, where $\theta=\frac{1}{2} \arctan \frac{J}{\epsilon_{2}-\epsilon_{1}}$ [7, 8]. The transformation to the eigenstate representation matrix is

$$
U^{-1}=\left(\begin{array}{rr}
-\sin \theta & \cos \theta \\
\cos \theta & \sin \theta
\end{array}\right) .
$$

The transition dipoles between the eigenstates and transition dipoles in the real space are related via one-excitonic and two-excitonic eigenvector matrices (Eqs. (125))

$$
\left(\begin{array}{l}
\mu_{e_{1} g} \\
\mu_{e_{2} g}
\end{array}\right)=\left(\begin{array}{rr}
\cos \theta & -\sin \theta \\
\sin \theta & \cos \theta
\end{array}\right)\left(\begin{array}{l}
\mathbf{d}_{1} \\
\mathbf{d}_{2}
\end{array}\right)
$$

and

$$
\left(\begin{array}{l}
\mu_{f e_{1}} \\
\mu_{f e_{2}}
\end{array}\right)=\left(\begin{array}{rr}
-\sin \theta & \cos \theta \\
\cos \theta & \sin \theta
\end{array}\right)\left(\begin{array}{l}
\mathbf{d}_{1} \\
\mathbf{d}_{2}
\end{array}\right)
$$

and squares of the transition dipoles between the eigenstates (oscillator strengths) are

$$
\begin{aligned}
& \left|\mu_{\mathrm{e}_{1} \mathrm{~g}}\right|^{2}=\left|\mu_{\mathrm{fe}_{1}}\right|^{2}=1+\frac{J \cos \phi}{\sqrt{\left(\epsilon_{2}-\epsilon_{1}\right)^{2}+J^{2}}}, \\
& \left|\mu_{\mathrm{e}_{2} \mathrm{~g}}\right|^{2}=\left|\mu_{\mathrm{fe}_{2}}\right|^{2}=1-\frac{J \cos \phi}{\sqrt{\left(\epsilon_{2}-\epsilon_{1}\right)^{2}+J^{2}}} .
\end{aligned}
$$

\subsection{Excitonically coupled dimer: signatures of} different evolution scenarios and pulse-overlap effects in $2 D$ spectra

Parameters used in calculations are $\epsilon_{1}=11800 \mathrm{~cm}^{-1}$ $\epsilon_{2}=12200 \mathrm{~cm}^{-1}, J=100 \mathrm{~cm}^{-1}, \phi=\frac{\pi}{6}$. The environment is represented by two overdamped Brownian oscillator coordinates, fast and slow, with relaxation rates $\Lambda_{\mathrm{F}}$ and $\Lambda_{\mathrm{S}}$, respectively. Both coordinates induce uncorrelated site-energy fluctuations as described above. We use the overdamped Brownian oscillator model where the spectral density of local chromophore energy fluctuations is [32]

$$
C^{\prime \prime}(\omega)=2 \sum_{l=\mathrm{S}, \mathrm{F}} \lambda_{l} \frac{\omega \Lambda_{l}}{\omega^{2}+\Lambda_{l}^{2}} .
$$

The corresponding lineshape function obtained by a direct double-time integral of Eq. (110) in the hightemperature limit [27, 29] is

$$
g(t)=\sum_{l=\mathrm{S}, \mathrm{F}} \lambda_{l} \frac{2 k_{\mathrm{B}} T-\mathrm{i} \Lambda_{l}}{\Lambda_{l}^{2}}\left(\mathrm{e}^{-\Lambda_{l} t}+\Lambda_{l} t-1\right)
$$

All system, bath, and coupling characterizing quantities are chosen typical of pigment molecules in photosynthetic proteins [34, 36]. Bath-induced fluctuations are described by overdamped $\mathrm{BO}$ parameters $\lambda_{\mathrm{F}}=$
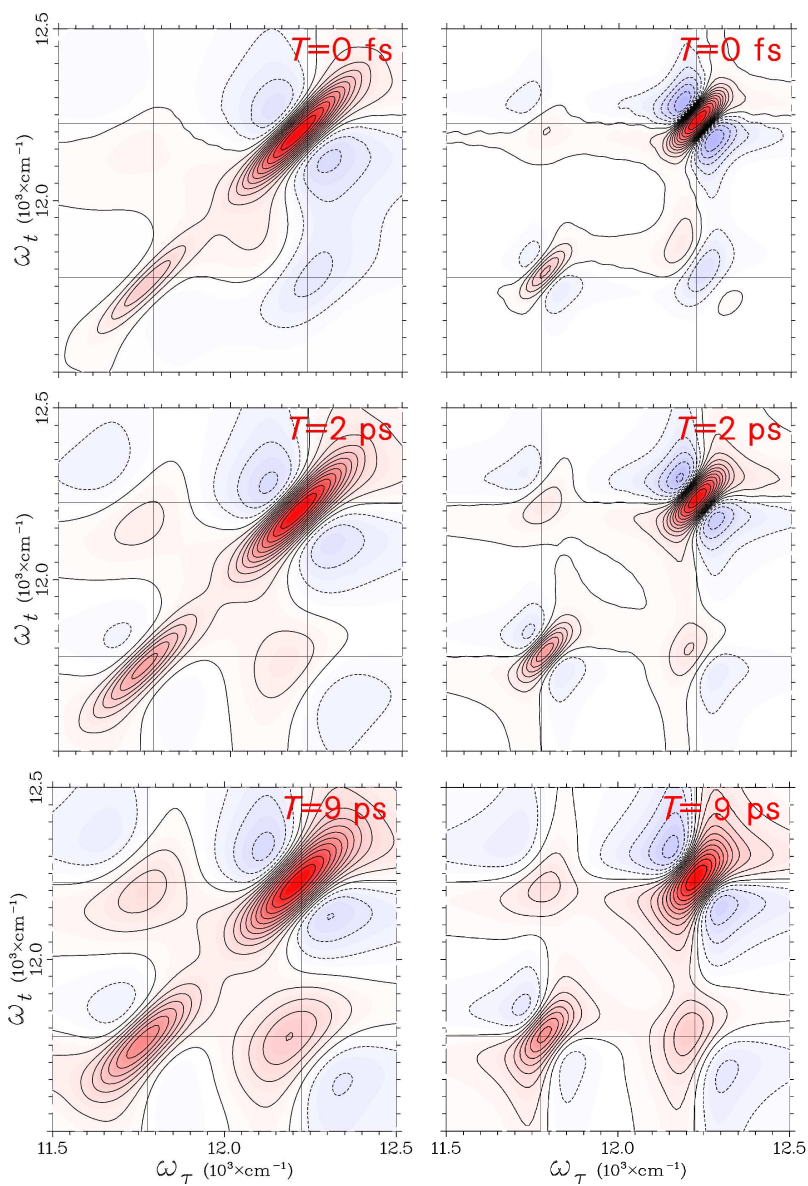

Fig. 10. 2D photon echo broad-bandwidth pulse signal at three delay times: $T=0,2$, and 9 ps. Left column is a broad-bandwidth ideal signal, right column is full signal reconstructed using the set of narrow-bandwidth simulations of a homo-dimer. See text for simulation parameters.

$30 \mathrm{~cm}^{-1}, \lambda_{\mathrm{S}}=60 \mathrm{~cm}^{-1}, \Lambda_{\mathrm{F}}^{-1}=50 \mathrm{fs}, \Lambda_{\mathrm{S}}^{-1}=10^{5} \mathrm{ps}$. The slow bath is used to model the static disorder, thus its fluctuation timescale $\Lambda_{\mathrm{S}}^{-1} \rightarrow \infty$. The calculated population transfer rates (Eq. (119)) for the eigenstates are: (downward) $K_{1 \leftarrow 2} / J=2.69$ and (upward) $K_{2 \leftarrow 1} / J=2.54 \cdot 10^{-3}$. The response function was then calculated as described in Sec. 3.4.

\subsubsection{Broad-bandwidth pulse simulations}

As a reference we first present the ideal impulsive 2D photon echo rephasing signal corresponding to the case of the short laser pulses when their spectral bandwidth is much larger than the width of the spectral region under consideration. In this regime the pulse overlap effect can be neglected and we obtain:

$$
W\left(\omega_{\tau}, T, \omega_{t}\right) \approx S_{\mathbf{k}_{\mathrm{I}}}\left(\omega_{\tau}, T, \omega_{t}\right),
$$

i. e. the signal probes the response function itself. We show such spectrum at two delay times in the left col- 

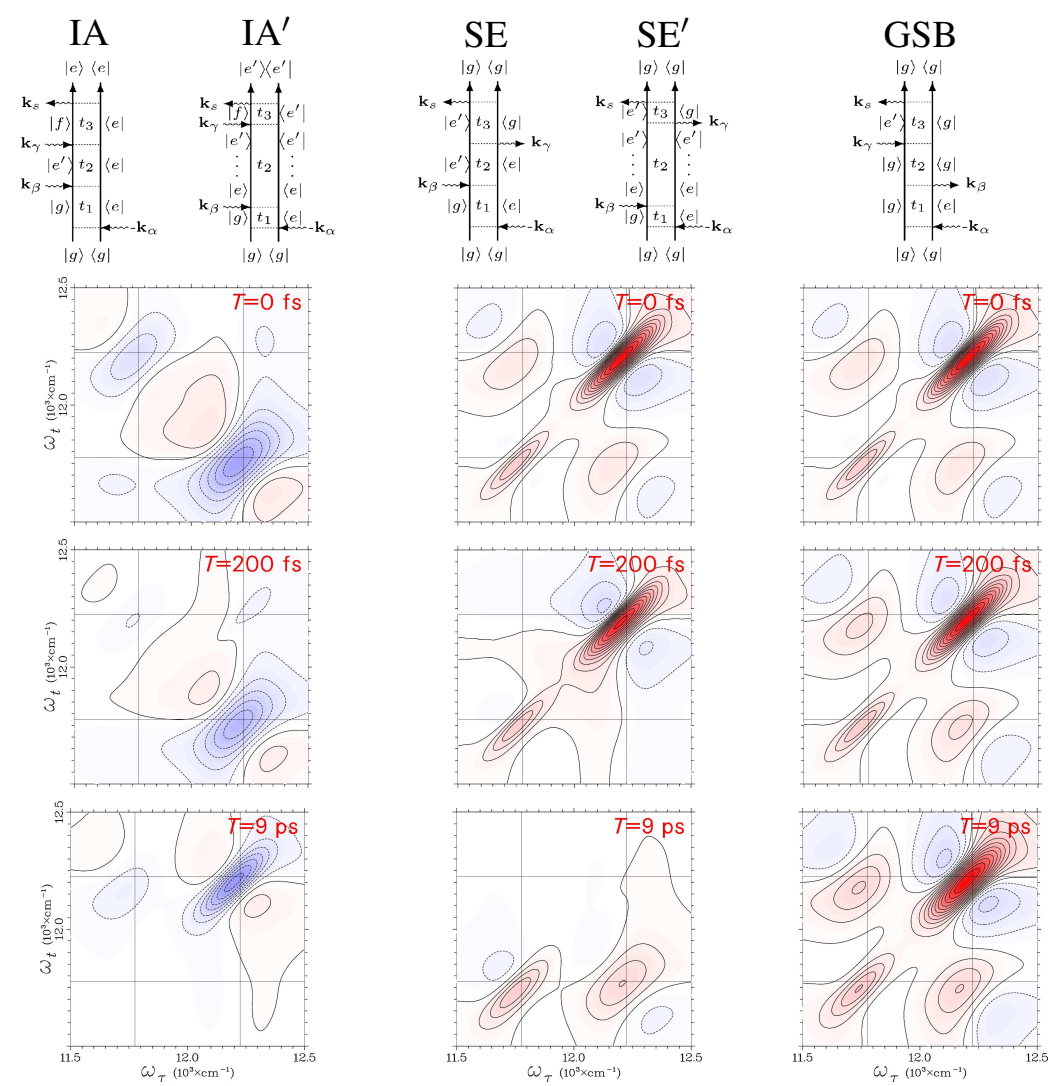

Fig. 11. The contributions of different LSPs to the real (absorptive) part of rephasing 2D spectra of excitonically coupled dimer at the impulsive limit; population transfer contributions IA $^{\prime}$ and SE' are merged with IA and SE; spectra are calculated at $T=0,200$ fs $(T<$ $\left.K_{1 \leftarrow 2}^{-1}\right)$, and 9 ps $\left(T_{2} \gg K_{1 \leftarrow 2}^{-1}\right)$. All graphs are normalized to the maximum of the most intensive contribution, contour lines are plotted using arcsinh scale.

umn of Fig. 10. The dissection of the spectra to components corresponding to different LSPs is presented in Fig. 11 for the real part of the rephasing signal, in Fig. 12 for the non-rephasing signal. The population transport diagrams are merged together with the coherent (no transport) diagrams in these figures. The spectra contain both diagonal and off-diagonal elements. Across the diagonal the peaks are broadened due to the homogeneous broadening caused by the fast term of bath oscillations. The lineshapes are extensively elongated along the diagonal due to the slow term of bath oscillations. The restriction $T_{2} \ll \Lambda_{\mathrm{S}}^{-1}$ ensures that the diagonal elongation remains for all delay times. Such approach is very efficient to model the inhomogeneous broadening, and represents the static disorder effect. Across the diagonal the peaks are broadened due to the homogeneous broadening caused by the fast term of bath oscillations.

At the short delay times $(T=0)$ the population transport is negligible and the diagonal peaks consist solely of the SE and GSB contributions. These two diagonal peaks represent two single-exciton eigenstates and are created when $e=e^{\prime}$, while the off-diagonal peaks correspond to $e \neq e^{\prime}$. At zero delay, the crosspeaks are created by the superposition of negative IA and positive GSB and SE contributions. At longer delay time we see the rise of the lower-energy peaks at $\omega_{t}=\omega_{\tau}=\varepsilon_{\mathrm{e}_{1}}$ demonstrating the down-hill population transfer in the excitonic system. Only IA and SE contributions change over population time $T$. GSB is conserved since there is no dynamics in the ground state contrary to the IA and SE diagrams, where the population dynamics during time delay $T$ is described by the $T$-dependent Green functions (population transfer) as well as coherence dephasing terms (Eqs. (112)-(114)). At long $T$ the diagonal IA and off-diagonal SE peaks come from population transport. The signal can be easily correlated with the Feynman diagrams.

Due to population transfer it is evident for $T=9 \mathrm{ps}$ that IA contribution refers to transition from the population first single-exciton state $\left|e_{1}\right\rangle\left\langle e_{1}\right|$ to the doubleexcited state $\left|f_{1}\right\rangle\left\langle e_{1}\right|$ solely. This is clearly indicated by a single off-diagonal element $\omega_{\tau}=\varepsilon_{e_{1}}, \omega_{t}=$ $\varepsilon_{f_{1}}-\varepsilon_{e_{1}} \approx \varepsilon_{e_{2}}$ for $T=9$ fs, while the off-diagonal peak for $\left|e_{2}\right\rangle\left\langle e_{2}|\rightarrow| f_{1}\right\rangle\left\langle e_{2}\right|$ transition is dominant for $T=0 \mathrm{fs}$ and $T=200 \mathrm{fs}$. The similar explanation 

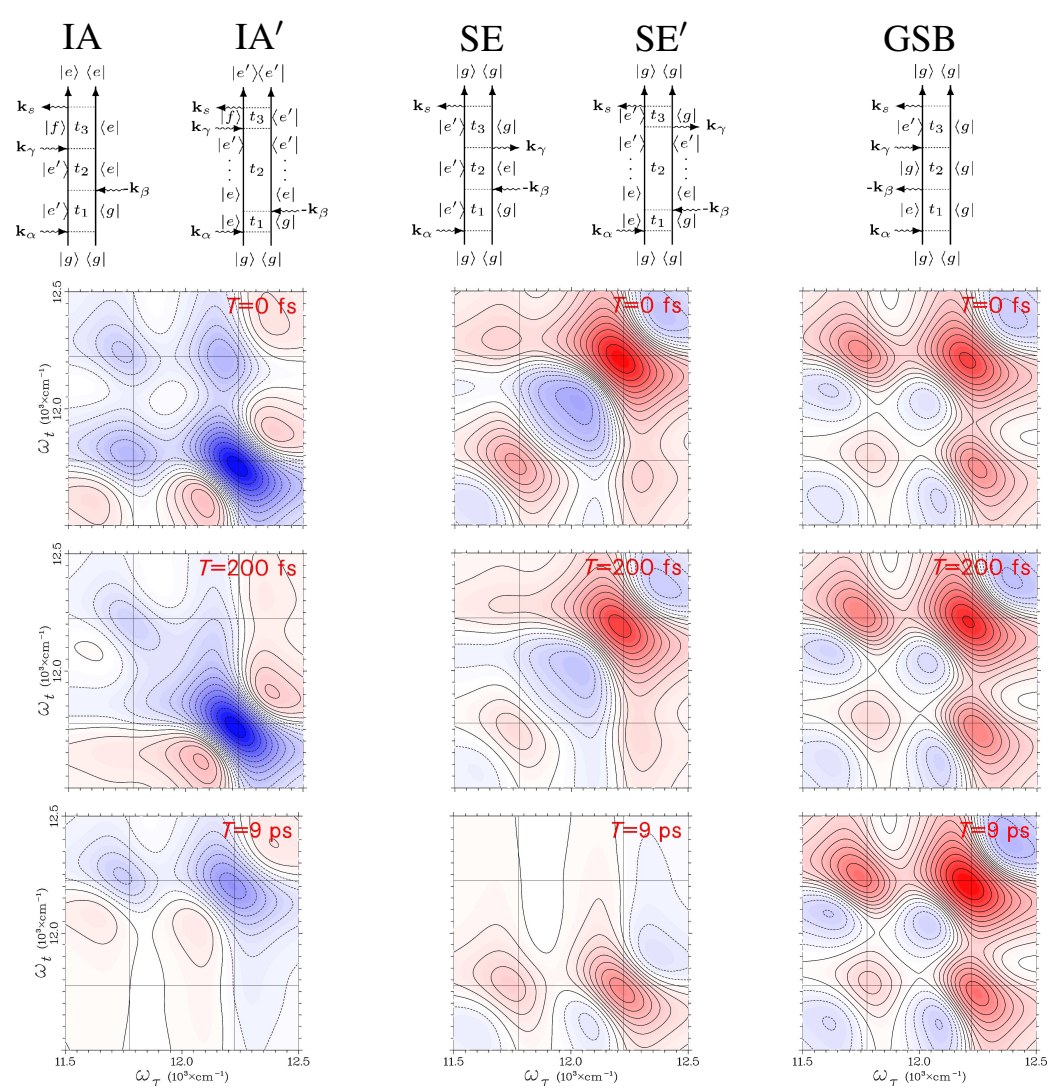

Fig. 12. The contributions of different LSPs to the real (absorptive) part of non-rephasing 2D spectra of excitonically coupled dimer in the impulsive limit. All parameters are analogous to Fig. 11.

holds for the population transfer-related off-diagonal peak that appears in SE diagram for $T=9$ ps. However, the double-exciton states are not included in SE diagram and the corresponding cross-peak is at $\omega_{\tau}=\varepsilon_{e_{2}}$, $\omega_{t}=\varepsilon_{e_{1}}$.

\subsubsection{Gaussian narrow-bandwidth simulations}

The finite-bandwidth Gaussian pulses $E_{j}(t), j=$ $1 \ldots 3$, (Eqs. (59)-(60) have two additional parameters: the carrier frequencies $\omega_{j}$ and pulse lengths $\left[\sigma_{t}\right]_{j}$. Changing the length of all pulses tunes the spectral bandwidths $\sigma_{\omega}^{-1}=\left[\sigma_{t}\right]_{j}$. Pulses with the increased length simulate the experiment more realistically, while the impulsive limit simulations are better for a purely phenomenological understanding of 2D spectra. Additionally, effects of pulse overlap arise [12]. Narrowbandwidth pulses also act as band-pass filters of 2D spectrograms [8]. The wavelenghts of laser pulses can be tuned independently to select certain resonances in the exciton system. By comparing the pulse bandwidths to the linewidth of a single peak in the spectra, we can obtain certain detection regimes. We assume that the pulse width is narrower than the whole exciton band-

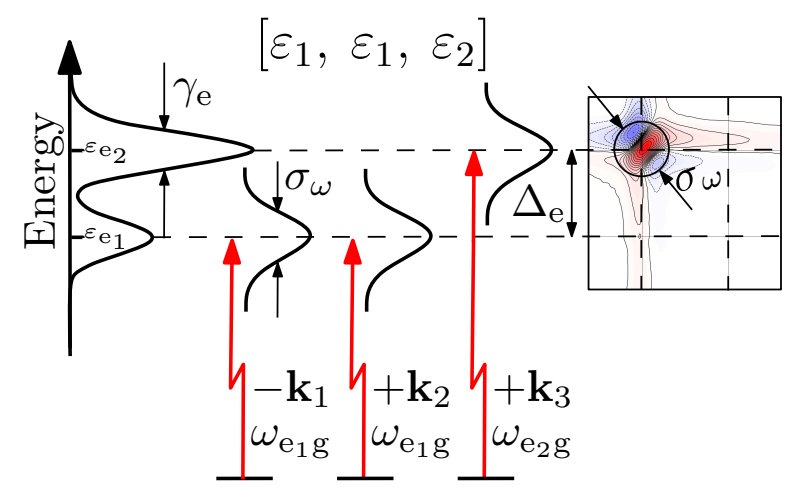

Fig. 13. Laser pulse wavelength tuning scheme for the FWM experiment. The wavelenghts of laser pulses are tuned independently to select certain resonances in the exciton system. In this example, first $\left(-\mathbf{k}_{1}\right)$ and second $\left(+\mathbf{k}_{2}\right)$ pulses have wavelengths, resonant to $\omega_{\mathrm{e}_{1} \mathrm{~g}}=\omega_{\mathrm{fe}_{2}}$ transition, while the third pulse $\left(+\mathbf{k}_{3}\right)$ is tuned to $\omega_{\mathrm{e}_{2} \mathrm{~g}}=\omega_{\mathrm{fe}_{1}}$. We use notation $\left[\varepsilon_{1}, \varepsilon_{1}, \varepsilon_{2}\right]$ for such configuration of laser frequencies.

width $\left(\sigma_{\omega}<\Delta_{e}\right)$, but broader than the width of a single peak $\left(\sigma_{\omega}>\gamma_{\mathrm{e}}\right)$ by setting $\sigma_{\omega}=1.2 \gamma_{e} \approx 0.16 \Delta_{e}$.

The model dimer has two single-exciton states with energies $\varepsilon_{\mathrm{e}_{1}}$ and $\varepsilon_{\mathrm{e}_{2}}$; the double-exciton state energy is $\varepsilon_{\mathrm{f}}=\varepsilon_{\mathrm{e}_{1}}+\varepsilon_{\mathrm{e}_{2}}$ (Eqs. (133)-(135)). The transition energies are $\omega_{\mathrm{fe}_{1}}=\varepsilon_{\mathrm{e}_{2}}$ and $\omega_{\mathrm{fe}_{2}}=\varepsilon_{\mathrm{e}_{1}}$. There- 

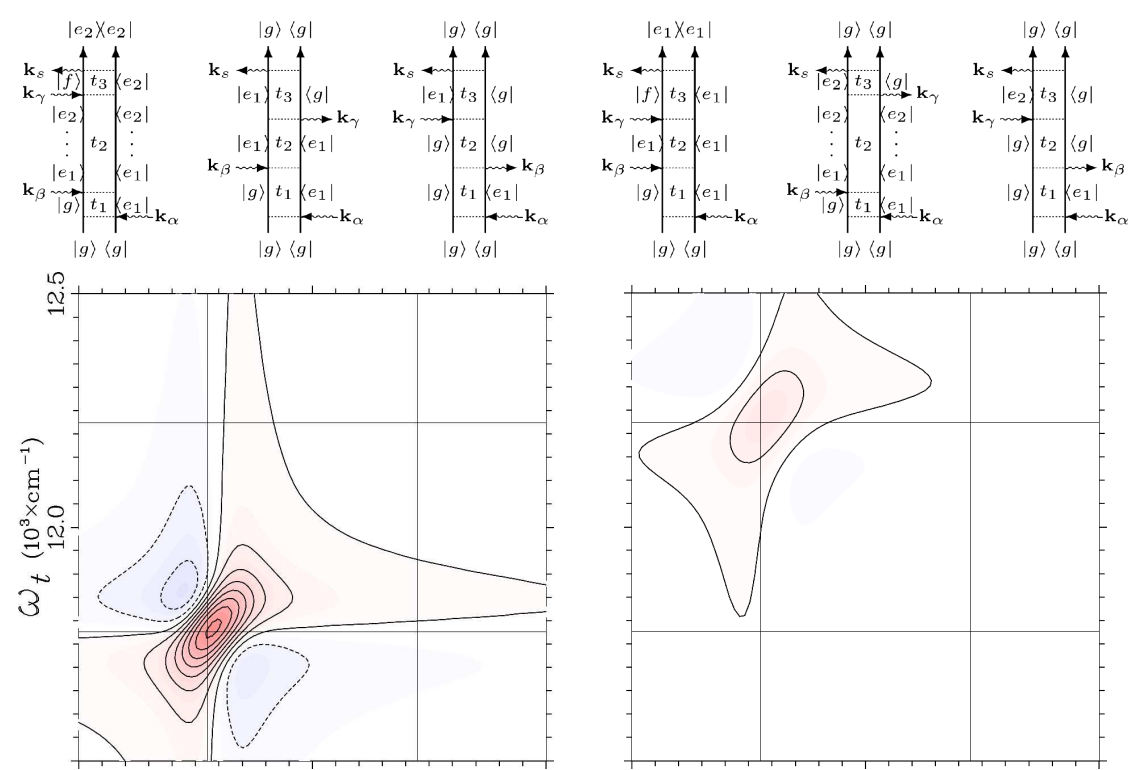

$\left[\varepsilon_{1}, \varepsilon_{1}, \varepsilon_{1}\right]$
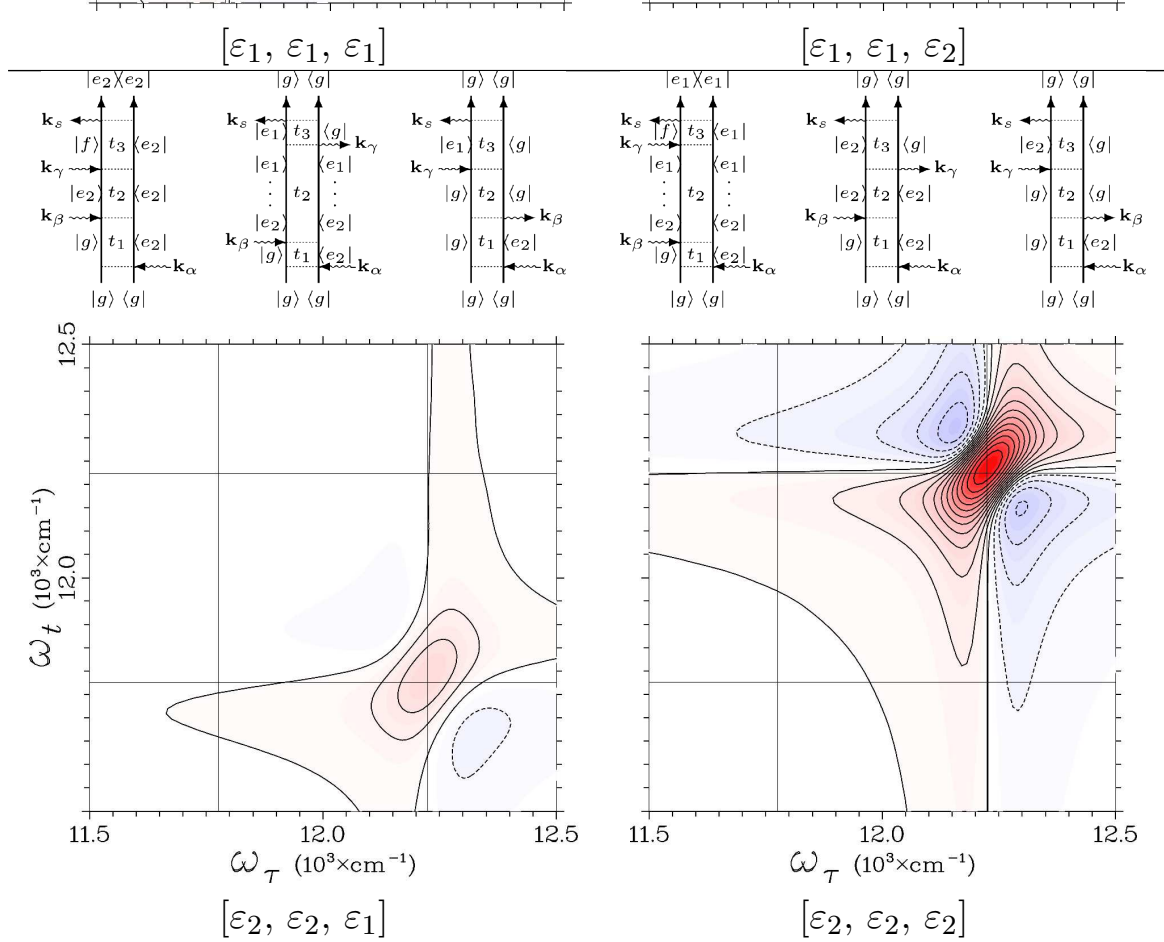

Fig. 14. Four most intensive narrow-bandwidth signals leading to resonant selection of Feynman diagrams in the signal at delay time $T=10 \mathrm{ps}$. The signals were simulated by varying central pulse frequencies while keeping the pulse bandwidths unchanged. Utilization of other possible laser pulse configurations gives negligible signals at $T \gg K_{1 \leftarrow 2}^{-1}$. All graphs are normalized to the global maximum $\left(\left[\varepsilon_{2}, \varepsilon_{2}, \varepsilon_{2}\right]\right.$ contribution).

fore, only two resonant pulse frequencies have to be considered. By considering all possible configurations of the carrier frequencies of incoming three pulses, and assuming that the fourth pulse (heterodyne) is broad ( $\delta$-shaped), we obtain $2^{3}=8$ possible permutations of the pulse frequencies, e.g. $\left[\omega_{1}, \omega_{2}, \omega_{3}\right]=$ $\left[\varepsilon_{1}, \varepsilon_{1}, \varepsilon_{1}\right],\left[\varepsilon_{1}, \varepsilon_{1}, \varepsilon_{2}\right] \ldots$ etc. This laser pulse wavelength tuning scheme is sketched in Fig. 13. However, once we select the resonant contributions, we find only six resonant configurations, four most significant of them are presented in Fig. 14 (by selecting the resonant pathways we have also considered population transport at non-zero delays $T$ ).

Appearance of specific spectral elements in manipulated spectra is controlled by laser pulse frequencies. The first laser pulse "controls" selection of spectral elements at $\omega_{\tau}$. For instance, in configurations with $\omega_{1}=\varepsilon_{\mathrm{e}_{1}}$, only spectral elements for $\omega_{\tau}=\omega_{\mathrm{e}_{1} \mathrm{~g}}$ do not 
vanish. The second pulse determines the state, which further evolves in range of $T$. The third pulse selects LSPs according to the resonant transitions during $T$.

It is remarkable that pulses select the distinct LSPs with high resolution. Various diagonal peaks and the crosspeaks now can be separately characterized including their shape and amplitude. Their time evolution follows the density matrix dynamics at corresponding LSPs. Some pulse frequency configurations are related to pure coherence pathways, which do not involve exciton populations $\left(\left[\varepsilon_{1}, \varepsilon_{2}, \varepsilon_{1}\right]\right.$ and $\left[\varepsilon_{2}, \varepsilon_{1}, \varepsilon_{2}\right]$ configurations, not shown). Other pathways filter out density matrix coherences and are initiated by the population and its transfer. The density matrix coherencecontributions show beating dynamics along $T$ (not shown), which follows the Schrödinger equation. The population contributions show monotonical dynamics due to classical dynamics according to the master equation.

The spectra on the right column of Fig. 10, reconstructed by summing up all the signals of different laser pulse configurations, resemble the broad-bandwidth signals (the left column of Fig. 10) very closely. By comparing the spectra it is noticeable that peaks of reconstructed broad-bandwidth spectra are slightly narrower due to the finite bandwidth of the pulses.

The pulse overlap effect in all simulations is small. That effect may be expected when one of the delay times $\tau$ or $t$ is smaller than the pulse duration $\sigma_{t}$. Then additional contributions follow: i) when pulse 1 is isolated but pulses 2 and 3 overlap and, thus, the term proportional to $E_{1}^{[1] *} E_{3}^{[2]} E_{2}^{[3]}$ contributes (this is an additional $S_{\mathbf{k}_{\mathrm{I}}}$ contribution), (ii) when pulse 3 is isolated and pulses 1 and 2 overlap providing with $E_{1}^{[2] *} E_{2}^{[1]} E_{3}^{[3]}$ contribution to the signal defined by the $S_{\mathbf{k}_{\text {II }}}$ term, and (iii) when all three pulses overlap, then all six terms in the integral contribute simultaneously. Fourier transformation, used in 2D signal construction, involves the integrations over delay times and the overlapping contributions are mixed with the non-overlapping contributions. If the response function is strong in pulse nonoverlapping regimes (usually longer delay times, which extend to infinity), then the non-overlapping contributions add up and dominate.

The overlap effect can be quantitatively characterized as follows. The response function of a dimer is characterized by two parameters: the splitting of the single-exciton states $\Delta_{\mathrm{e}}$ and the characteristic linewidth of each single-exciton resonance $\gamma$. The time-domain response functions then experience splitting-related oscillations with frequency $\Delta_{\mathrm{e}}$ and the decay with timescale $\gamma_{\mathrm{e}}^{-1}$. In our case $\Delta_{\mathrm{e}}>\gamma_{\mathrm{e}}$ and we observe wellseparated exciton resonances. The ideal impulsive conditions are fulfilled when $\sigma_{\omega} \gg \Delta_{\mathrm{e}}$ and $\sigma_{\omega} \gg \gamma_{\mathrm{e}}$. This corresponds to the impulsive regime of ultrashort pulses, when their overlaps can be neglected. For realistic finite-bandwidth Gaussian pulses we need to consider pulse durations, $\sigma_{\omega}^{-1}$. In two dimensions of time $(\tau, t)$, the whole area, where the response function is not zero, is $\gamma_{\mathrm{e}}^{-2}$. The area, where pulses overlap, is $\sigma_{\omega}^{-2}$. The ratio $\eta=\sigma_{\omega}^{-2} / \gamma_{\mathrm{e}}^{-2}$, thus, characterizes the relative pulse-overlap magnitude. Our finite-bandwidth simulations are in the regime $\gamma_{\mathrm{e}}<\sigma_{\omega}<\Delta_{\mathrm{e}}$. We have $\eta<1$ and the pulses can thus specifically select resonant peaks, the response function decays slowly compared to the pulse duration, and, therefore, the pulseoverlaps make a very small contribution. We call this regime quasi-impulsive. That is the ideal regime to be used for selection of specific pathways of the system with well-separated peaks. The ideal impulsive experiment can then be reconstructed from a set of narrowbandwidth measurements.

\subsection{Dimer of three-level chromophores}

Let us consider a homo-dimer of three-level chromophores, that is, two coupled anharmonic oscillators, described in Sec. 3.3. Molecular Hamiltonian of the system is

$$
\begin{aligned}
\hat{H}_{\mathrm{mol}}= & \omega_{0} \sum_{m=1}^{2} \hat{a}_{m}^{\dagger} \hat{a}_{m}+J \sum_{m \neq n}^{2} \hat{a}_{m}^{\dagger} \hat{a}_{n} \\
& +\frac{\Delta}{2} \sum_{m=1}^{2} \hat{a}_{m}^{\dagger} \hat{a}_{m}^{\dagger} \hat{a}_{m} \hat{a}_{m} .
\end{aligned}
$$

The one-exciton block is identical to a homo-dimer of two-level systems

$$
\hat{h}^{(1)}=\left(\begin{array}{cc}
\omega_{0} & J \\
J & \omega_{0}
\end{array}\right)
$$

and corresponding exciton energies and eigen-vectors are described by Eqs. (133)-(136). In site representation the two-exciton block is built by adding states $\hat{a}_{1}^{\dagger} \hat{a}_{1}^{\dagger}|0\rangle$ and $\hat{a}_{2}^{\dagger} \hat{a}_{2}^{\dagger}|0\rangle$ with energies $2 \omega_{0}+\Delta$ due to the overtone states of single chromophores. The coupling constant between the overtone states and multi-exciton state with energy $2 \omega_{0}$ is therefore equal to $\sqrt{2} J$ :

$$
\hat{h}^{(2)}=\left(\begin{array}{ccc}
2 \omega_{0}+\Delta & \sqrt{2} J & 0 \\
\sqrt{2} J & 2 \omega_{0} & \sqrt{2} J \\
0 & \sqrt{2} J & 2 \omega_{0}+\Delta
\end{array}\right) .
$$


Diagonalization of one- and two-exciton blocks (Eqs. (122)-(123)) allows us to move to the exciton basis

$$
\begin{aligned}
& \left|e_{j}\right\rangle=\sum_{m=1}^{N} U_{j m}^{-1} \hat{a}_{m}^{\dagger}|0\rangle \\
& \left|f_{k}\right\rangle=\sum_{m=1}^{N} \sum_{n=m}^{N} \nu_{m n}^{(k)}\left(\zeta_{m n}+\frac{\delta_{m n}}{\sqrt{2}}\right) \hat{a}_{m}^{\dagger} \hat{a}_{n}^{\dagger}|0\rangle
\end{aligned}
$$

where $\nu_{m n}^{(k)}$ denotes the element of $V^{-1}$ of the $k$ th row and the column, corresponding to the diagonal element of $\hat{a}_{m}^{\dagger} \hat{a}_{n}^{\dagger}|0\rangle$ state and $N=2$. The one-exciton eigenvector matrix is identical to Eq. (136). The two-exciton eigenvector matrix is

$$
V^{-1}=\frac{1}{\sqrt{2}}\left(\begin{array}{ccc}
\frac{\sin \vartheta}{2 \cos \frac{\vartheta}{2}} & \frac{-\cos \vartheta-1}{\sqrt{2} \cos \frac{\vartheta}{2}} & \frac{\sin \vartheta}{2 \cos \frac{\vartheta}{2}} \\
1 & 0 & -1 \\
\frac{\sin \vartheta}{2 \sin \frac{\vartheta}{2}} & \frac{-\cos \vartheta+1}{\sqrt{2} \sin \frac{\vartheta}{2}} & \frac{\sin \vartheta}{2 \sin \frac{\vartheta}{2}}
\end{array}\right)
$$

where $\vartheta=\arctan \left(\frac{4 J}{\Delta}\right)$. The eigen-energies of one- and two-exciton states are

$$
\begin{aligned}
& \varepsilon_{e_{1}}=\omega_{0}+J, \\
& \varepsilon_{e_{2}}=\omega_{0}-J,
\end{aligned}
$$

and

$$
\begin{aligned}
& \varepsilon_{f_{1}}=2 \omega_{0}+2 J \frac{\cos \vartheta-1}{\sin \vartheta}, \\
& \varepsilon_{f_{2}}=2 \omega_{0}+\Delta, \\
& \varepsilon_{f_{3}}=2 \omega_{0}+2 J \frac{\cos \vartheta+1}{\sin \vartheta} .
\end{aligned}
$$

Dipole moments for the ground to one- and twoexciton states are

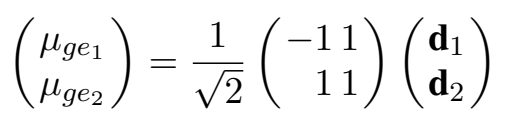

and

$$
\left(\begin{array}{l}
\mu_{e_{1} f_{1}} \\
\mu_{e_{1} f_{2}} \\
\mu_{e_{1} f_{3}}
\end{array}\right)=\frac{1}{\sqrt{2}}\left(\begin{array}{cc}
-\frac{\sin \vartheta+\cos \vartheta+1}{2 \cos \frac{\vartheta}{2}} & \frac{\sin \vartheta+\cos \vartheta+1}{2 \cos \frac{\vartheta}{2}} \\
-1 & -1 \\
-\frac{\sin \vartheta+\cos \vartheta-1}{2 \sin \frac{\vartheta}{2}} & \frac{\sin \vartheta+\cos \vartheta-1}{2 \sin \frac{\vartheta}{2}}
\end{array}\right)\left(\begin{array}{l}
\mathbf{d}_{1} \\
\mathbf{d}_{2}
\end{array}\right)
$$

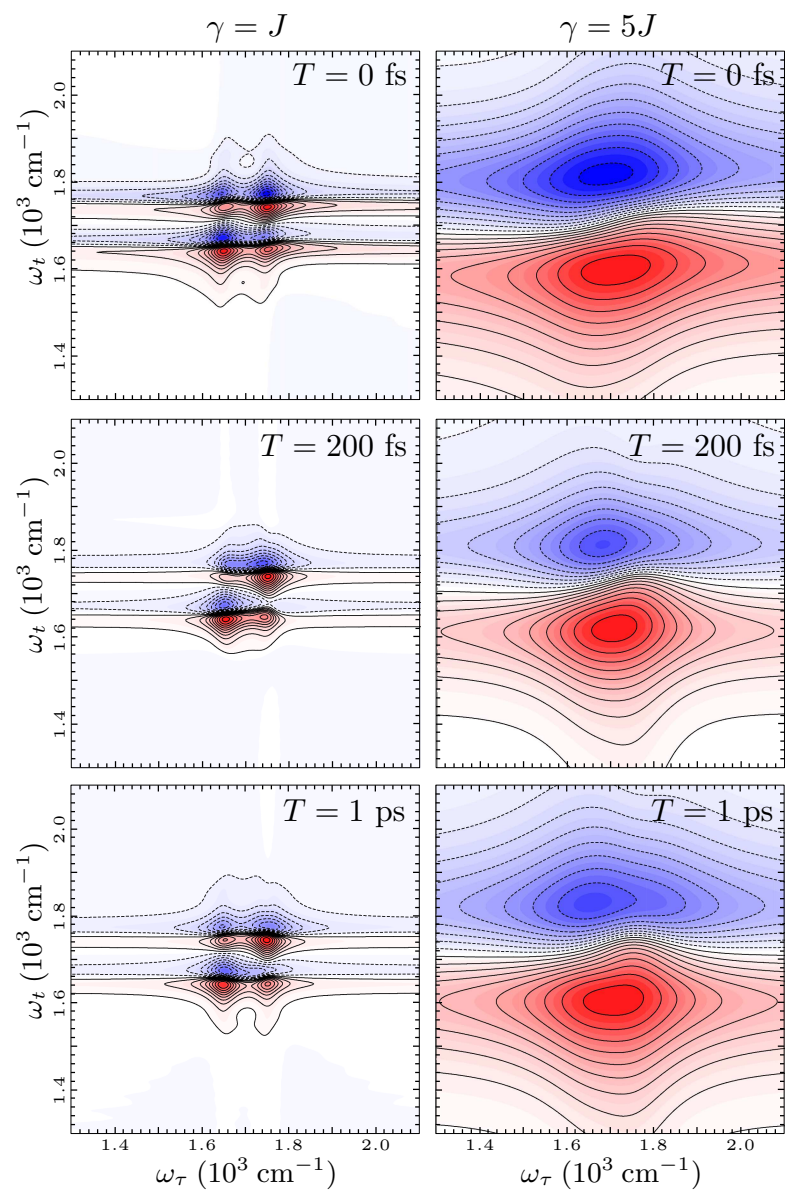

Fig. 15. The real part of total 2D spectra of the dimer of three-level system with dephasing constant $\gamma=J$ (left column) and $\gamma=5 J$ (right column). $J=50 \mathrm{~cm}^{-1}$ and $\Delta=15 \mathrm{~cm}^{-1}$ in both cases.

and

$$
\left(\begin{array}{l}
\mu_{e_{2} f_{1}} \\
\mu_{e_{2} f_{2}} \\
\mu_{e_{2} f_{3}}
\end{array}\right)=\frac{1}{\sqrt{2}}\left(\begin{array}{cc}
\frac{\sin \vartheta-\cos \vartheta-1}{2 \cos \frac{\vartheta}{2}} & \frac{\sin \vartheta-\cos \vartheta-1}{2 \cos \frac{\vartheta}{2}} \\
1 & -1 \\
\frac{\sin \vartheta-\cos \vartheta+1}{2 \sin \frac{\vartheta}{2}} & \frac{\sin \vartheta-\cos \vartheta+1}{2 \sin \frac{\vartheta}{2}}
\end{array}\right)\left(\begin{array}{l}
\mathbf{d}_{1} \\
\mathbf{d}_{2}
\end{array}\right) .
$$

Having transition dipole moments defined one would be able to construct analytic expressions of the total 2D signal from individual Feynman diagrams. In Fig. 15 the spectra of the dimer of the three-level system using different dephasing rates are presented. For a dimer of two-level systems, coupling of the eigenstates is represented by off-diagonal elements. Coherent induced absorption diagrams produce spectral elements on the diagonal and gives oscillations of corresponding diagonal peaks, that is perceptible in 2D spectra at the limit of $\gamma=J$. However, if the dephasing rate is $\gamma \gg J$, the inter-state coherence dynamics cannot be separated from diagonal elements and the whole spectra resemble a single anharmonic oscillator. 


\section{Applications to multi-chromophore complexes}

\subsection{One-dimensional J-aggregates of pseudoisocyanine}

Molecular aggregates (J-aggregates) are macroscopic clusters of molecules with intermolecular spacing intermediate between the crystal lattice and isolated molecules. The scientific interest in spectroscopic features of such structures remains high even since 1936 when Jelley [37] and Scheibe [38] independently discovered a significant narrowing of the absorption band, red shifted relative to a monomer band, due to an increase of the concentration of the dye pseudoisocyanine (PIC) in water solution. This narrowing effect upon an aggregation is known as the superradiance.

The J-band in the streaming solution is polarized along the streaming direction (see, for instance, [39]) and thus the transition dipole moments of the constituent molecules of the linear one-dimensional Jaggregate must have a small angle with respect to the aggregate axis. In this case the optical transition to the lowest exciton states dominates in the absorption spectrum, while the narrowing of its lineshape is addressed to the motional narrowing [40, 41]. Linear one-dimensional J-aggregates are formed by parallel molecules with an angle of transition dipoles less than $\arccos \sqrt{1 / 3} \approx 54.7^{\circ}$ with respect to the aggregate axis. In the other case an $\mathrm{H}$-aggregate is formed with the superradiant fluorescence feature at the highest energy.

Spectral properties of J-aggregates are usually understood in terms of the Frenkel exciton theory [42]. The exciton energy spectrum and the corresponding wave functions are defined from diagonalization of the exciton Hamiltonian of a linear chain of $N$ identical molecules

$\hat{H}_{\mathrm{mol}}=\sum_{m=1}^{N}\left(\epsilon+\delta \epsilon_{m}\right)|m\rangle\left\langle m\left|+\sum_{m=1}^{N} \sum_{n \neq m}^{N} J_{n m}\right| n\right\rangle\langle m|$,

where $\epsilon$ is the excitation energy of a constituent molecule and $\delta \epsilon_{m}$ is the random (inhomogeneous) Gaussiandistributed energy offset of the $m$ th molecule, $|n\rangle$ denotes the state when the $n$th molecule in the aggregate is excited and $\langle n|$ is its Hermitian conjugate. Matrix elements $J_{n m}$ denote the energies of the resonance interaction between the $n$th and $m$ th molecules, which can be calculated from the structural data. In the absence of the diagonal disorder (when $\delta \epsilon_{m} \equiv 0$ ) and in the case of the nearest-neighbour coupling approximation (assuming that $J_{m n}=-J_{0} \delta_{|n-m|, 1}$ with $J_{0} \geq 0$ ), the exciton Hamiltonian defined by Eq. (158) can be diagonalized analytically resulting, thus, for the eigenenergies [34, 42] in

$$
\varepsilon_{j}=\varepsilon-2 J_{0} \cos \frac{\pi j}{N+1}
$$

and eigenfunctions $\left|e_{j}\right\rangle=\sum_{n} \psi_{j n}|n\rangle$, where

$$
\psi_{j n}=\sqrt{\frac{2}{N+1}} \sin \frac{\pi j n}{N+1} .
$$

Quantum numbers $j=1 \ldots N$ enumerate the exciton states. The transition dipole moments corresponding to each exciton state are defined giving the dipole strength for a particular exciton state as follows:

$$
\left(\mu_{j}\right)^{2}=\frac{2 \mu_{0}^{2}}{N+1} \cot ^{2} \frac{\pi j}{2(N+1)}
$$

for odd $j$ and

$$
\left(\mu_{j}\right)^{2}=0
$$

for even $j$, where $\mu_{0}$ denominates the transition dipole moment of a constituent molecule from the aggregate. Thus, the dipole strength of the lowest energy state $(j=1)$, which is red-shifted in comparison with the molecular transition in accord with Eq. (159), is significantly higher than that of the others and contains more than $80 \%$ of the total oscillator strength.

The absorption spectrum of pseudoisocyanine (PIC) $\mathrm{J}$-aggregates features not only the J-band (at $576.5 \mathrm{~nm}$ ) but two weak bands at 536 and $499 \mathrm{~nm}$ as well. While the origin of the J-band is clear, this cannot be said about the other two bands. Conflicting theories have been put forward but there is no consensus yet. On the basis of detailed analysis of the absorption and fluorescence excitation spectra, the one-dimensional model containing four molecules per unit cell was proposed [43]. According to this model the unit cell of the aggregate consists of four PIC molecules and, within the dipole approximation, the transition dipoles in the unit cell are of the following orientation: $\left(\mu_{x}, \mu_{y}, \mu_{z}\right)$, $\left(-\mu_{x},-\mu_{y}, \mu_{z}\right),\left(\mu_{x},-\mu_{y}, \mu_{z}\right)$, and $\left(-\mu_{x}, \mu_{y}, \mu_{z}\right)$, where $\mu=\left(\mu_{x}, \mu_{y}, \mu_{z}\right)=(0.581,0.338,0.738)$ (Fig. 16). These transition dipole moments cause the average value of the coupling constant between the nearest neighbours $J_{0}$ being equal to -1.43 in units of $|\mu|^{2} /\left(r_{12}\right)^{3}$, where $r_{12}$ is the translation vector norm. An experimentally observed exciton bandwidth is achieved by setting $|\mu|^{2} /\left(r_{12}\right)^{3}$ to reach $J_{0}=$ $600 \mathrm{~cm}^{-1}$.

To showcase the narrowing of the J-band, we present simulations of absorption and 2D total (rephasing + 


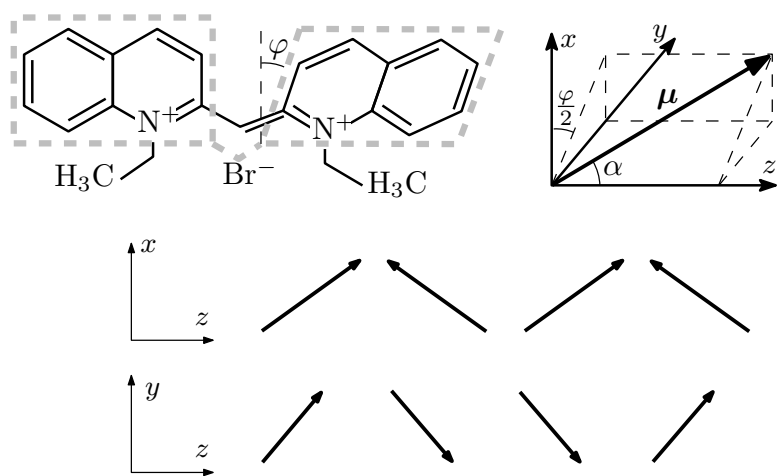

Fig. 16. The spatial structure of a single unit cell consisting of four PIC molecules of a J-aggregate used in the calculations. The transition dipole moment vector is denoted as $\mu$ and their corresponding projections to $x 0 z$ and $y 0 z$ planes are depicted. The dihedral angle $\varphi$ and tilt angle $\alpha$ are known from spectroscopic measurements [43].

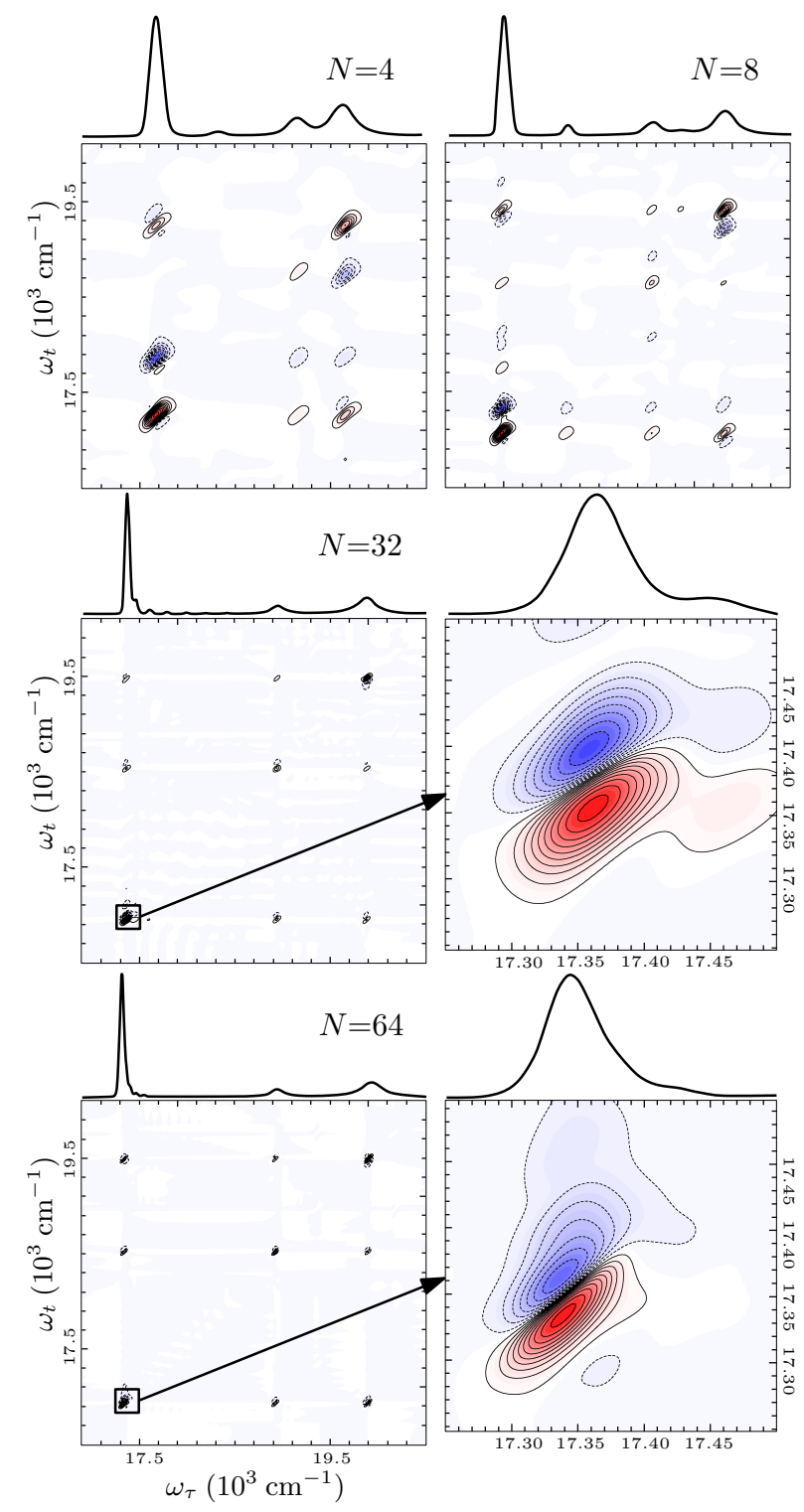

Fig. 17. Absorption spectra and the real part of total 2D spectra of PIC J-aggregate with different number of chromophores $N$. non-rephasing) spectra of J-aggregates consisting of different number of molecules (Fig. 17). Absorption spectra simulations of aggregates of more than 64 molecules did not contain any noticeable differences. All 2D spectra are presented at population time $T=$ 0 fs. To simulate inhomogeneous broadening we first use random Gaussian off-set terms $\delta \epsilon_{m}$ in Eq. (158) and average over 1000 distributions to get the absorption spectra. We set diagonal disorder to $\sigma_{\mathrm{D}}=60 \mathrm{~cm}^{-1}$ that is 0.1 of the coupling constant $J_{0}$. Then we fit the parameters of ,slow“ mode of spectral density (Eq. (141)) to match the aforementioned absorption spectra. The simulation of inhomogeneous broadening in 2D spectra is then accomplished using the "slow" mode thus allowing us to avoid computationally expensive numerical diagonalization of multiple instances of the Frenkel exciton Hamiltonian.

Let us first consider the absorption spectrum of a single unit cell consisting of four molecules. Four bands are visible in the absorption spectrum (even though the transition to the second excitonic state is very weak, the corresponding peak is still visible). The J-band is at the bottom of the single exciton manifold. The peaks representing the fourth and third excitonic states are visible as well.

The 2D spectra of the unit cell contain more information. The J-band is clearly dominant here as well. However, the second excitonic state is not visible, which is due to the fact that peak intensities are proportional to the fourth order of transition dipole moments in the 2D spectrum and to the second order in the absorption spectrum. As expected, the peaks corresponding to the third and fourth excitonic states are also visible on the diagonal. The positive crosspeaks represent coherences and correlations between excitonic states. The negative elements, that are due to induced absorption, contain information about two-exciton states.

Now let us consider the effects of aggregation by analysing the difference in absorption and 2D spectra of J-aggregates consisting of an increasing number of molecules. Firstly, we can see the considerable narrowing of the J-band. Obviously, with the increasing number of molecules, the oscillator strength corresponding to the J-band increases. This effect is more easily seen in 2D spectra, where the spectral elements become so narrow that it is basically impossible to analyse the 2D spectra of J-aggregates consisting of 64 or even 32 molecules when it is plotted on the same scale as the 2D spectra of the unit cell. Moreover, the presence of the negative IA contribution distorts the appearance of the $\mathrm{J}$-band in the 2D spectra in case of 32 or 64 molecules 
in the aggregate. Secondly, it can be clearly seen from both absorption and 2D spectra that the width of the excitonic manifold increases with the aggregate size. The increase is not linear, however, as the width increase slows quickly with increasing aggregate size. Finally, it can be seen that the J-band in the absorption spectrum becomes slightly assymetric when the aggregate consists of 64 molecules. To highlight this we show two insets in Fig. 17. In case of the 32-molecule aggregate, the J-band is still symmetric but in case of the 64-molecule aggregate it becomes assymetric due to the fact that the third excited state has some oscillator strength and its peak blends with the main J-band peak corresponding to the first excited state. As can be clearly seen from the insets, the J-band is elongated along the $\omega_{\tau}$ axis. This elongation is due to the crosspeak representing the $\left|e_{1}\right\rangle\left\langle e_{3}\right|$ coherence. This elongation, however, is clearly smaller in case of the 64-molecule aggregate. Therefore, from analysing the 2D spectra we can conclude that the third excited state is getting nearer to the first one and it should be noticeable in the absorption spectra.

\subsection{Excitation transfer pathways in}

\section{Fenna-Matthews-Olson photosynthetic complex}

The Fenna-Matthews-Olson (FMO) protein is a photosynthetic light harvesting complex found in some bacterias. In the process of photosynthesis, it has a role in energy transfer between the light-absorbing antennae (chlorosomes) and the reaction centre [34, 36]. The FMO complex has three identical subunits, each of them containing 7 bacteriochlorophyll- $a$ molecules (Fig. 18). This complex was the first bacteriochlorophyll containing protein to have its structure determined with a very high resolution $(2.2 \AA)$ by means of X-ray spectroscopy [44]. It was extracted from the green photosynthetic bacteria Chlorobium tepidum.

A highly-effective energy transfer was observed experimentally in this complex. It is assumed that it is related to the long-lived electronic coherences ( $>600 \mathrm{fs}$ ), that were observed by means of $2 \mathrm{D}$ electronic spectroscopy [14]. The energy is believed to be transferred in a wave-like manner by two dominant pathways. The energy transfer through electronic coherences should ensure high efficiency [11, 15, 45]. However, traditional quantum relaxation theories often fail in describing such a slow coherence decay in electronic systems of strong excitonic coupling [46-48].

Intensive researches of the FMO complex are undergoing since its structure is the simplest of all known

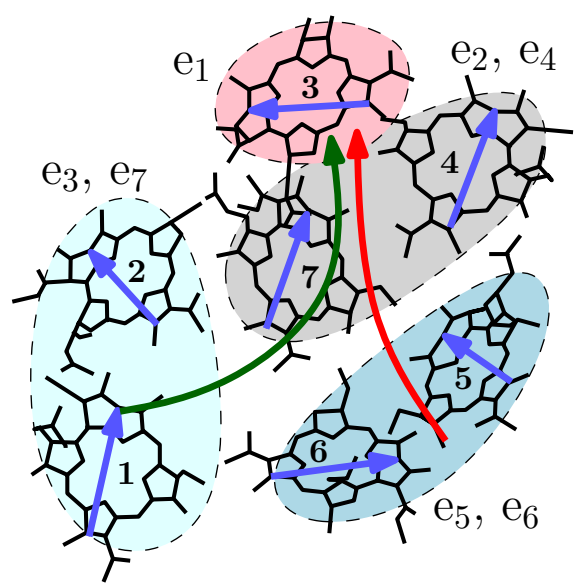

Fig. 18. The structure of Fenna-Matthews-Olson complex. Chromophore numbers as well as exciton states are identified. Arrows illustrate excitation transfer pathways within the system (revealed in further discussion).

photosynthetic proteins. A single subunit has the same optical properties as the whole complex. The absorption band is narrow and linear absorption simulation using the Frenkel exciton theory provides very good results.

In this paper, simulations of $2 \mathrm{D}$ spectra of the FMO photosynthetic complex are presented. Dipole moments were calculated from the structural data of the complex [49]. Molecular Hamiltonian and relaxation rates are the same as in Ref. [3]. System-bath interaction is represented by the fast and slow modes of overdamped Brownian oscillator, as in Sec. 5.2: $\lambda_{\mathrm{F}}=$ $30 \mathrm{~cm}^{-1}, \lambda_{\mathrm{S}}=60 \mathrm{~cm}^{-1}, \Lambda_{\mathrm{F}}^{-1}=100 \mathrm{fs}, \Lambda_{\mathrm{S}}^{-1}=$ $10^{5}$ ps., similar to refs. [12, 50]. Temperature is $77 \mathrm{~K}$.

\subsubsection{Spatial delocalization of molecular excitons}

In Eqs. (128) three different correlation functions were obtained, involving either single-exciton states, double-exciton states, or single- and double-exciton states. The wave function overlap terms have different physical meanings, representing either degree of delocalization, or spatial overlaps of molecular excitons within the complex.

First, let us consider the $C_{\mathrm{e}_{j} \mathrm{e}_{j} \mathrm{e}_{j} \mathrm{e}_{j}}^{\prime \prime}(\omega)$ term. The wave function overlap contribution represents the mean square of the single-exciton state fluctuations $\left\langle\delta \Omega_{j}^{2}\right\rangle$,

$$
\sum_{m} U_{m j}^{4}=N_{j}^{-1},
$$

where $N_{j}$ is so-called inverse participation ratio (IPR). Value of the IPR shows, how many molecules are involved in the $j$ th exciton, so it is the measure of the exciton delocalization. If the exciton state is completely localized, $N_{j}=1$. At the other limit, when the $j$ th 
Table 1. Squares of matrix elements of eigen-vector matrix $U$, inverse participation ratios (IPR), and transition dipole moment squares $\left|\mu_{g e_{j}}\right|^{2}$. The most significant values are in bold.

\begin{tabular}{cccccccc}
\hline & \multicolumn{7}{c}{ Single-exciton state } \\
& $\mathrm{e}_{1}$ & $\mathrm{e}_{2}$ & $\mathrm{e}_{3}$ & $\mathrm{e}_{4}$ & $\mathrm{e}_{5}$ & $\mathrm{e}_{6}$ & $\mathrm{e}_{7}$ \\
\hline BChl 1 & 0.00 & 0.00 & $\mathbf{0 . 7 8}$ & 0.00 & 0.00 & 0.00 & $\mathbf{0 . 2 2}$ \\
BChl 2 & 0.00 & 0.00 & $\mathbf{0 . 2 2}$ & 0.00 & 0.00 & 0.00 & $\mathbf{0 . 7 8}$ \\
BChl 3 & $\mathbf{0 . 8 8}$ & 0.11 & 0.00 & 0.01 & 0.00 & 0.00 & 0.00 \\
BChl 4 & 0.11 & $\mathbf{0 . 6 0}$ & 0.00 & 0.10 & 0.13 & 0.06 & 0.00 \\
BChl 5 & 0.01 & 0.11 & 0.00 & 0.11 & $\mathbf{0 . 4 5}$ & $\mathbf{0 . 3 2}$ & 0.00 \\
BChl 6 & 0.00 & 0.01 & 0.00 & 0.03 & $\mathbf{0 . 4 2}$ & $\mathbf{0 . 5 4}$ & 0.00 \\
BChl 7 & 0.00 & 0.17 & 0.00 & $\mathbf{0 . 7 6}$ & 0.00 & 0.07 & 0.00 \\
IPR & 1.28 & 2.45 & 1.53 & 1.67 & 2.54 & 2.47 & 1.53 \\
$\left|\mu_{g e_{j}}\right|^{2}$ & 14.8 & 26.4 & 22.2 & 7.9 & 27.8 & 6.3 & 11.2 \\
\hline
\end{tabular}

exciton is completely delocalized in a $N$-chromophore system, $U_{m j}=N^{-1 / 2}$, that is, the mean square of the single-exciton state fluctuations becomes very small, $\left\langle\delta \Omega_{j}^{2}\right\rangle \propto N^{-1}$, this exchange-narrowing effect is typical of molecular J-aggregates. Squares of matrix elements of $U$ represent localizations of excitons in each molecule. For the FMO complex, calculated matrix elements and IPRs, as well as corresponding transition dipole moment squares, are presented in Table 1 .

For other correlation functions in Eqs. (128), $C_{\mathrm{e}_{j} \mathrm{e}_{j} \mathrm{e}_{k} \mathrm{e}_{k}}^{\prime \prime}(\omega)$ represents the spatial overlap of probability density functions of $j$ th and $k$ th excitonic state (squares of eigen-functions in excitonic basis). Correlation functions involving double-excites states have similar physical meaning [3].

In Table 1 we present calculated square values of the eigenvector matrix, square values of transition dipole moments, and inverse participation ratios. It is notable that IPR values are small. This shows that the molecular excitations are not extensively delocalized. The first excitonic state $e_{1}$ is mostly localized in the third chromophore. $e_{2}$ and $e_{4}$ excitons are commonly localized in 4 and 7 chromophores, $e_{3}$ and $e_{7}$ in 1 and 2 chromophores, $e_{5}$ and $e_{6}$ in 5 and 6 chromophores. The largest square values of transition dipole moments are $\left|\mu_{g e_{2}}\right|^{2}$ and $\left|\mu_{g e_{5}}\right|^{2}$, the corresponding absorption peaks dominate both in absorption and 2D spectra.

\subsubsection{Energy transfer pathways}

By analysing the evolution of 2D spectra and knowing how the excitons are localized spatially in the complex, we are able to describe the excitation transfer pathways within the system.

In the calculations, $\delta$-shaped laser pulses were used. It means that all transitions between excitonic levels can be induced. Simulated spectra of FMO complex for various population times $T$ are presented in Fig. 19. It
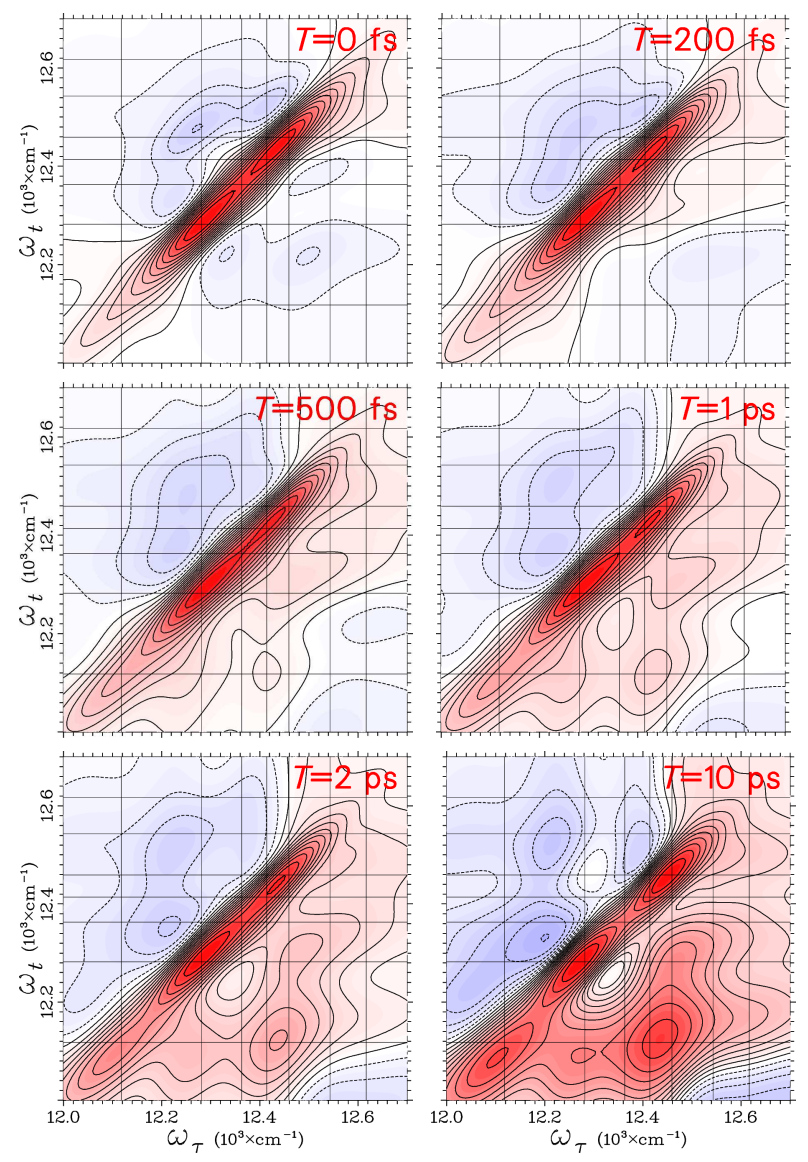

Fig. 19. Real (absorptive) part of rephasing 2D spectra of photosynthetic FMO complex.

is remarkable that the results fit the experimental data well $[3,13,25,31]$. While increasing the population time $T$, some features of $2 \mathrm{D}$ spectra change: diagonal elements decay except for the 1-3 and 5 exciton states. The corresponding off-diagonal elements denote the energy transfer from the higher energy molecular excitations downwards, $e_{4}, e_{5} \rightarrow e_{1}$ and $e_{2} \rightarrow e_{1}$. So, the excitation, induced in some region of the complex, will be transferred to chromophores 4 and 7, and then to chromophore 1. Therefore, two pathways of excitation transfer can be distinguished: $e_{3}, e_{7} \rightarrow e_{2}, e_{4} \rightarrow e_{3}$ and $e_{5}, e_{6} \rightarrow e_{2}$. They are demonstrated in Fig. 18 by the left and right (green and red online) curved arrows, respectively.

The total signal can be decomposed to pathways, indicated by the double-sided Feynman diagrams, as it was shown for the excitonically coupled dimer in Sec. 5.2.1. The corresponding spectra are presented in Fig. 20. 


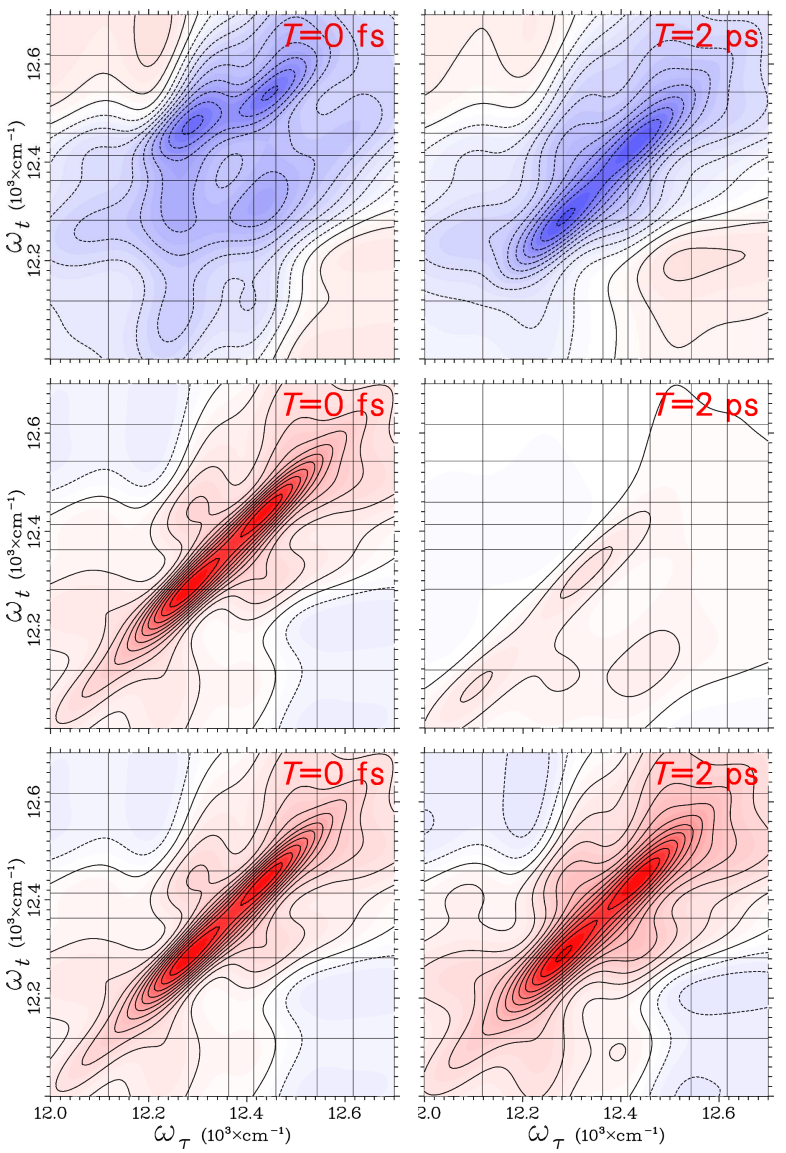

Fig. 20. Components of 2D spectra of the FMO complex at $T=0$ and 2 ps: IA (first row), SE (second row), GSB (third row).

\section{Concluding remarks}

The basic concepts of two-dimensional optical spectroscopy are presented in this study. By performing the four-wave mixing experiment, the detected photonecho signal fully represents the third-order polarization dynamics induced within the system and separation of quantum coherence and population dynamics, homogeneous and inhomogeneous broadening is possible. Such sophisticated experimental method adds up one more dimension to the time-resolved spectroscopic measurement with respect to the other nonlinear spectroscopic techniques. Therefore, it allows us to directly observe ultra-fast quantum phenomena such as excitonic energy transfer and quantum coherence dynamics in time-resolved two-dimensional spectrograms. Utilization of so-called two-colour two-dimensional optical spectroscopy also provides means of implementing quantum coherence control of the relevant system as well as specific excitation pathway induction.

To illustrate the main features of the two-dimensional optical spectroscopy a number of examples are considered in this study. The examples are presented consistently from the simpliest quantum two- and threelevel systems to the molecular excitonic aggregates.

For the basic quantum systems the total third-order response signal can be expressed analytically by assuming simple models of the solvent dynamics. The simulated two-dimensional spectra of a two-level atom (Lorentzian spectral lineshape) and a two-level molecule (Gaussian spectral lineshape) are presented and various representations are discussed. For an anharmonic oscillator (three-level system) evidences of anharmonicity are considered.

Two coupled chromophores constitute a more sophisticated system of a dimer, in the spectra of which the quantum coherence dynamics is observed. Two types of the dimer description are presented: the constituting chromophores are considered as two-level or three-level subsystems. In the case of the two-level chromophore hetero-dimer, extraction of individual spectral elements by means of two-colour two-dimensional spectroscopy is demonstrated. It is possible to characterize the dipole moment configuration and resonant coupling constant value by performing such measurements. For the homo-dimer of three-level chromophores, complete expressions for the transition to the excitonic eigenstate basis are derived and time-resolved two-dimensional spectra are presented. In case of low dephasing rate, spectra demonstrate typical spectral features of anharmonic nature, while in the limit of the dephasing rate being much higher than the intrinsic system dynamics the total spectra are not clearly separable from the spectra of a single three-level system.

Finally, in the analysis of complex molecular aggregates all the powerful capabilities of this spectroscopic tool can be demonstrated. For the two-dimensional spectra of linear one-dimensional J-aggregates the spectrally narrowed peak due to motional-narrowing, the J-band, demonstrates a great complexity of the spectral behaviour due to different excitation pathways producing spectral elements of different time-resolved dynamics in the vicinity of the J-band. Analysis of this spectral region helps to distinguish the mechanisms of excitation transfer and nuclear-motion induced motional-narrowing and consider the timescales of these processes within the aggregate. The formation of the J-band with respect to the aggregate size is presented and analysed in this paper. The excitation transfer dynamics in the photosynthetic light-harvesting Fenna-Matthews-Olson complex is comprehensively described in this study. The systematic separation of the spectral elements with respect to the corresponding physical mechanisms is carried out and the dominant 
highly-efficient excitation transfer pathways within the system are identified.

\section{Acknowledgements}

This research was supported by the global grant of the Research Council of Lithuania according to Operational Programme for Human Resource Development and the Research Council of Lithuania Student Research Fellowship Award (V. B., A. G.)

\section{Appendices}

\section{A. The cumulant expansion}

The cumulant expansion technique is widely used to simplify expressions of perturbational series in time. Assume that we have a perturbational series of some quantity $A$

$$
A(t)=1+\lambda A_{1}(t)+\lambda^{2} A_{2}(t)+\ldots
$$

and want to recast it into the exponential form

$$
A(t) \equiv \exp \left[\lambda F_{1}(t)+\lambda^{2} F_{2}(t)+\ldots\right] .
$$

If the Taylor expansion of exponential is carried out and terms of the same $\lambda$ order are collected, the relation between $F_{i}$ and $A_{j}$ terms is obtained:

$$
\begin{aligned}
A(t) & =\left(1+\lambda F_{1}+\frac{1}{2} \lambda^{2} F_{1}^{2}+\frac{1}{6} \lambda^{3} F_{1}^{3}+\ldots\right) \\
& \times\left(1+\lambda^{2} F_{2}+\ldots\right)\left(1+\lambda^{3} F_{3}+\ldots\right) \\
& =1+\lambda F_{1}+\lambda^{2}\left(\frac{1}{2} F_{1}^{2}+F_{2}\right) \\
& +\lambda^{3}\left(\frac{1}{6} F_{1}^{3}+F_{1} F_{2}+F_{1} F_{3}\right)+\ldots
\end{aligned}
$$

For the cumulant expansion to the second order we have

$$
A(t)=\exp \left\{\lambda A_{1}(t)+\lambda^{2}\left[A_{2}(t)-\frac{1}{2} A_{1}(t)\right]+\ldots\right\}
$$

The cumulant expansion is also a way to represent a truncation of perturbation series. If we truncate Eq. (A1) to the values of a certain order, we still have an infinite series in exponential of Eq. (A4). On the other hand, we can sketch that we have Eq. (A1) calculated to the terms of infinite order and truncate the cumulant expansion to the certain length, containing only terms of the lowest order.

For the second-order cumulant expansion of timeordered exponentials of energy-gap correlation functions, i. e.

$$
\begin{aligned}
& A(t)=\exp _{+}\left[-\mathrm{i} \int_{t_{0}}^{t} \mathrm{~d} \tau \Delta V(\tau)\right] \\
& =1-\mathrm{i} \int_{t_{0}}^{t} \mathrm{~d} \tau \Delta V(\tau)+\mathrm{i}^{2} \int_{t_{0}}^{t} \mathrm{~d} \tau \int_{t_{0}}^{\tau} \mathrm{d} \tau^{\prime} \Delta V(\tau) \Delta V\left(\tau^{\prime}\right),
\end{aligned}
$$

for the expectation value of $A(t)$ we obtain

$$
\langle A(t)\rangle=\exp \left\{-\int_{t_{0}}^{t} \mathrm{~d} \tau \int_{t_{0}}^{\tau} \mathrm{d} \tau^{\prime}\left\langle\Delta V(\tau) \Delta V\left(\tau^{\prime}\right)\right\rangle\right\}
$$

\section{B. Response function of an open excitonic system with diagonal and off-diagonal fluctuations}

Here we present the third-order response function expression written out in terms of Feynman diagrams, shown in Fig. 8. We are considering the $\mathbf{k}_{\mathrm{I}}$ experimental technique, where the spatial configuration of incident pulse wave vectors is $-\mathbf{k}_{1}+\mathbf{k}_{2}+\mathbf{k}_{3}[21]$. The pulses are generally not $\delta$-shaped, therefore the pulseoverlap effects are present, that mix the $\mathbf{k}_{\mathrm{I}}$ signal with the signals of other experimental techniques.

$\mathbf{k}_{\mathrm{I}}=-\mathbf{k}_{\alpha}+\mathbf{k}_{\beta}+\mathbf{k}_{\gamma}$ diagrams

$S^{\mathrm{IA}_{\mathrm{c}}}=-\sum_{e^{\prime} \neq e} \sum_{f}\left\langle\mu_{e^{\prime}} \mu_{e^{\prime} f} \mu_{e f} \mu_{e}\right\rangle$

$$
\begin{aligned}
& \times \exp \left\{\mathrm{i} \omega_{e g} t_{1}-\mathrm{i} \omega_{e^{\prime} e} t_{2}-\mathrm{i} \omega_{f e} t_{3}\right. \\
& +\operatorname{conj}\left[-g_{e^{\prime} e^{\prime}}^{*}\left(t_{2}\right)-g_{f f}^{*}\left(t_{3}\right)-g_{e e}\left(t_{1}+t_{2}+t_{3}\right)\right. \\
& -g_{e^{\prime} f}^{*}\left(t_{2}+t_{3}\right)+g_{e^{\prime} f}^{*}\left(t_{2}\right)+g_{e^{\prime} f}^{*}\left(t_{3}\right) \\
& -g_{e^{\prime} e}\left(t_{1}\right)+g_{e^{\prime} e}^{*}\left(t_{2}+t_{3}\right)+g_{e^{\prime} e}\left(t_{1}+t_{2}\right)-g_{e^{\prime} e}^{*}\left(t_{3}\right) \\
& \left.\left.-g_{e f}\left(t_{1}+t_{2}\right)+g_{e f}^{*}\left(t_{3}\right)+g_{e f}\left(t_{1}+t_{2}+t_{3}\right)\right]\right\}
\end{aligned}
$$


$S^{\mathrm{IA}_{\mathrm{p}}}=-\sum_{e^{\prime}=e} \sum_{f} G_{e^{\prime} e}\left(t_{2}\right)\left\langle\left|\mu_{e^{\prime}}\right|^{2}\left|\mu_{e^{\prime} f}\right|^{2}\right\rangle$

$$
\begin{aligned}
& \times \exp \left\{\mathrm{i} \omega_{e g} t_{1}-\mathrm{i} \omega_{f e} t_{3}\right. \\
& +\operatorname{conj}\left[-g_{e^{\prime} e^{\prime}}^{*}\left(t_{2}\right)-g_{f f}^{*}\left(t_{3}\right)-g_{e e}\left(t_{1}+t_{2}+t_{3}\right)\right. \\
& -g_{e^{\prime} f}^{*}\left(t_{2}+t_{3}\right)+g_{e^{\prime} f}^{*}\left(t_{2}\right)+g_{e^{\prime} f}^{*}\left(t_{3}\right) \\
& -g_{e^{\prime} e}\left(t_{1}\right)+g_{e^{\prime} e}^{*}\left(t_{2}+t_{3}\right)+g_{e^{\prime} e}\left(t_{1}+t_{2}\right)-g_{e^{\prime} e}^{*}\left(t_{3}\right) \\
& \left.\left.-g_{e f}\left(t_{1}+t_{2}\right)+g_{e f}^{*}\left(t_{3}\right)+g_{e f}\left(t_{1}+t_{2}+t_{3}\right)\right]\right\}
\end{aligned}
$$

$$
S^{\mathrm{SE}_{c}}=\sum_{e \neq e^{\prime}}\left\langle\left|\mu_{e}\right|^{2}\left|\mu_{e^{\prime}}\right|^{2}\right\rangle
$$

$$
\begin{aligned}
& \times \exp \left\{\mathrm{i} \omega_{e g} t_{1}+\mathrm{i} \omega_{e e^{\prime}} t_{2}-\mathrm{i} \omega_{e^{\prime}} t_{3}\right. \\
& -g_{e e}^{*}\left(t_{1}+t_{2}\right)-g_{e^{\prime} e^{\prime}}\left(t_{2}+t_{3}\right) \\
& \left.-g_{e e^{\prime}}^{*}\left(t_{1}\right)+g_{e e^{\prime}}^{*}\left(t_{1}+t_{2}+t_{3}\right)+g_{e e^{\prime}}\left(t_{2}\right)-g_{e e^{\prime}}^{*}\left(t_{3}\right)\right\}
\end{aligned}
$$$$
S^{\mathrm{SE}_{\mathrm{p}}}=\sum_{e=e^{\prime}} G_{e^{\prime} e}\left(t_{2}\right)\left\langle\left|\mu_{e}\right|^{2}\left|\mu_{e^{\prime}}\right|^{2}\right\rangle
$$

$$
\begin{aligned}
& \times \exp \left\{\mathrm{i} \omega_{e g} t_{1}-\mathrm{i} \omega_{e^{\prime} g} t_{3}\right. \\
& -g_{e e}^{*}\left(t_{1}+t_{2}\right)-g_{e^{\prime} e^{\prime}}\left(t_{2}+t_{3}\right) \\
& \left.-g_{e e^{\prime}}^{*}\left(t_{1}\right)+g_{e e^{\prime}}^{*}\left(t_{1}+t_{2}+t_{3}\right)+g_{e e^{\prime}}\left(t_{2}\right)-g_{e e^{\prime}}^{*}\left(t_{3}\right)\right\}
\end{aligned}
$$$$
S^{\mathrm{SE}^{\prime}}=\sum_{e \neq e^{\prime}} G_{e^{\prime} e}\left(t_{2}\right)\left\langle\left|\mu_{e}\right|^{2}\left|\mu_{e^{\prime}}\right|^{2}\right\rangle
$$$$
\times \exp \left\{-\mathrm{i} \omega_{e g} t_{1}+\mathrm{i} \omega_{e^{\prime} g} t_{3}-\gamma_{e^{\prime}} t_{3}-\gamma_{e} t_{1}\right.
$$$$
-g_{e e}\left(t_{1}\right)-g_{e^{\prime} e^{\prime}}^{*}\left(t_{3}\right)+g_{e^{\prime} e}\left(t_{1}+t_{2}+t_{3}\right)
$$$$
\left.-g_{e^{\prime} e}\left(t_{1}+t_{2}\right)-g_{e^{\prime} e}\left(t_{2}+t_{3}\right)+g_{e^{\prime} e}\left(t_{2}\right)\right\}
$$

$$
\begin{aligned}
& S^{\mathrm{GSB}}=\sum_{e e^{\prime}}\left\langle\left|\mu_{e}\right|^{2}\left|\mu_{e^{\prime}}\right|^{2}\right\rangle \exp \left\{\mathrm{i} \omega_{e g} t_{1}-\mathrm{i} \omega_{e^{\prime} g} t_{3}\right. \\
& \quad-g_{e e}^{*}\left(t_{1}\right)-g_{e^{\prime} e^{\prime}}\left(t_{3}\right)-g_{e e^{\prime}}^{*}\left(t_{1}+t_{2}\right) \\
& \left.\quad+g_{e e^{\prime}}^{*}\left(t_{1}+t_{2}+t_{3}\right)+g_{e e^{\prime}}^{*}\left(t_{2}\right)-g_{e e^{\prime}}^{*}\left(t_{2}+t_{3}\right)\right\}
\end{aligned}
$$

$$
\mathbf{k}_{\mathrm{II}}=+\mathbf{k}_{\alpha}-\mathbf{k}_{\beta}+\mathbf{k}_{\gamma} \text { diagrams }
$$

$$
\begin{aligned}
& S^{\mathrm{I} \mathrm{A}_{\mathrm{p}}}=-\sum_{e^{\prime}=e} \sum_{f} G_{e^{\prime} e}\left(t_{2}\right)\left\langle\left|\mu_{e^{\prime} f}\right|^{2}\left|\mu_{e}\right|^{2}\right\rangle \\
& \quad \times \exp \left\{-\mathrm{i} \omega_{e^{\prime} g} t_{1}+\mathrm{i} \omega_{e f} t_{3}\right. \\
& +\operatorname{conj}\left[-g_{e^{\prime} e^{\prime}}^{*}\left(t_{1}+t_{2}\right)-g_{f f}^{*}\left(t_{3}\right)-g_{e e}\left(t_{2}+t_{3}\right)\right. \\
& -g_{e^{\prime} f}^{*}\left(t_{1}+t_{2}+t_{3}\right)+g_{e^{\prime} f}^{*}\left(t_{1}+t_{2}\right)+g_{e^{\prime} f}^{*}\left(t_{3}\right) \\
& -g_{e^{\prime} e}^{*}\left(t_{1}\right)+g_{e^{\prime} e}^{*}\left(t_{1}+t_{2}+t_{3}\right)+g_{e^{\prime} e}\left(t_{2}\right)-g_{e^{\prime} e}^{*}\left(t_{3}\right) \\
& \left.\left.-g_{f e}\left(t_{2}\right)+g_{f e}^{*}\left(t_{3}\right)+g_{f e}\left(t_{2}+t_{3}\right)\right]\right\}
\end{aligned}
$$

$$
S^{\mathrm{IA}^{\prime}}=-\sum_{e^{\prime} \neq e} \sum_{f} G_{e^{\prime} e}\left(t_{2}\right)\left\langle\left|\mu_{e^{\prime} f}\right|^{2}\left|\mu_{e}\right|^{2}\right\rangle
$$

$$
\begin{aligned}
& \times \exp \left\{\mathrm{i} \omega_{e g} t_{1}-\mathrm{i} \omega_{e^{\prime} f} t_{3}-\gamma_{e^{\prime}} t_{3}-\gamma_{e} t_{1}\right. \\
& +\operatorname{conj}\left[-g_{e e}\left(t_{1}\right)-g_{f f}\left(t_{3}\right)-g_{e^{\prime} e^{\prime}}^{*}\left(t_{3}\right)\right. \\
& -g_{f e}\left(t_{1}+t_{2}+t_{3}\right)+g_{f e}\left(t_{1}+t_{2}\right)+g_{f e}\left(t_{2}+t_{3}\right) \\
& +g_{e^{\prime} e}\left(t_{1}+t_{2}+t_{3}\right)-g_{e^{\prime} e}\left(t_{1}+t_{2}\right)-g_{e^{\prime} e}\left(t_{2}+t_{3}\right) \\
& \left.\left.+g_{e^{\prime} f}\left(t_{3}\right)+g_{f e^{\prime}}^{*}\left(t_{3}\right)+g_{e^{\prime} e}\left(t_{2}\right)-g_{f e}\left(t_{2}\right)\right]\right\}
\end{aligned}
$$

$$
\begin{aligned}
& S^{\mathrm{I} \mathrm{I}_{\mathrm{c}}}=-\sum_{e^{\prime} \neq e} \sum_{f}\left\langle\mu_{e^{\prime}} \mu_{e^{\prime} f} \mu_{f e} \mu_{e}\right\rangle \\
& \quad \times \exp \left\{-\mathrm{i} \omega_{e^{\prime} g} t_{1}+\mathrm{i} \omega_{e e^{\prime}} t_{2}+\mathrm{i} \omega_{e f} t_{3}\right. \\
& +\operatorname{conj}\left[-g_{e^{\prime} e^{\prime}}^{*}\left(t_{1}+t_{2}\right)-g_{f f}^{*}\left(t_{3}\right)-g_{e e}\left(t_{2}+t_{3}\right)\right. \\
& -g_{e^{\prime} f}^{*}\left(t_{1}+t_{2}+t_{3}\right)+g_{e^{\prime} f}^{*}\left(t_{1}+t_{2}\right)+g_{e^{\prime} f}^{*}\left(t_{3}\right) \\
& -g_{e^{\prime} e}^{*}\left(t_{1}\right)+g_{e^{\prime} e}^{*}\left(t_{1}+t_{2}+t_{3}\right)+g_{e^{\prime} e}\left(t_{2}\right)-g_{e^{\prime} e}^{*}\left(t_{3}\right) \\
& \left.\left.-g_{f e}\left(t_{2}\right)+g_{f e}^{*}\left(t_{3}\right)+g_{f e}\left(t_{2}+t_{3}\right)\right]\right\}
\end{aligned}
$$




$$
\begin{aligned}
& \mathbf{k}_{\mathrm{III}}=+\mathbf{k}_{\alpha}+\mathbf{k}_{\beta}-\mathbf{k}_{\gamma} \text { diagrams } \\
& S^{\mathrm{SE}_{\mathrm{p}}}=\sum_{e^{\prime}=e} G_{e^{\prime} e}\left(t_{2}\right)\left\langle\left|\mu_{e}\right|^{2}\left|\mu_{e^{\prime}}\right|^{2}\right\rangle \\
& \times \exp \left\{-\mathrm{i} \omega_{e g} t_{1}-\mathrm{i} \varepsilon_{e^{\prime} g} t_{3}\right. \\
& -g_{e e}^{*}\left(t_{2}\right)-g_{e^{\prime} e^{\prime}}\left(t_{1}+t_{2}+t_{3}\right)-g_{e e^{\prime}}\left(t_{1}\right) \\
& \left.+g_{e e^{\prime}}^{*}\left(t_{2}+t_{3}\right)+g_{e e^{\prime}}\left(t_{1}+t_{2}\right)-g_{e e^{\prime}}^{*}\left(t_{3}\right)\right\} \\
& S^{2 \mathrm{Q}_{1}}=\sum_{e^{\prime} e} \sum_{f}\left\langle\mu_{e^{\prime}} \mu_{e^{\prime} f} \mu_{f e} \mu_{e}\right\rangle \\
& \times \exp \left\{-\mathrm{i} \omega_{e g} t_{1}-\mathrm{i} \omega_{f g} t_{2}-\mathrm{i} \omega_{e^{\prime} g} t_{3}\right. \\
& -g_{e^{\prime} e^{\prime}}\left(t_{3}\right)-g_{f f}\left(t_{2}\right)-g_{e e}\left(t_{1}\right)-g_{e^{\prime} f}\left(t_{2}+t_{3}\right) \\
& +g_{e^{\prime} f}\left(t_{3}\right)+g_{e^{\prime} f}\left(t_{2}\right)-g_{e^{\prime} e}\left(t_{1}+t_{2}+t_{3}\right) \\
& +g_{e^{\prime} e}\left(t_{2}+t_{3}\right)+g_{e^{\prime} e}\left(t_{1}+t_{2}\right)-g_{e^{\prime} e}\left(t_{2}\right) \\
& \left.-g_{f e}\left(t_{1}+t_{2}\right)+g_{f e}\left(t_{2}\right)+g_{f e}\left(t_{1}\right)\right\} \\
& S^{\mathrm{SE}^{\prime}}=\sum_{e^{\prime} \neq e} G_{e^{\prime} e}\left(t_{2}\right)\left\langle\left|\mu_{e}\right|^{2}\left|\mu_{e^{\prime}}\right|^{2}\right\rangle \\
& \times \exp \left\{-\mathrm{i} \omega_{e g} t_{1}-\mathrm{i} \omega_{e^{\prime} g} t_{3}-\gamma_{e^{\prime}} t_{3}-\gamma_{e} t_{1}\right. \\
& -g_{e e}\left(t_{1}\right)-g_{e^{\prime} e^{\prime}}\left(t_{3}\right)-g_{e^{\prime} e}\left(t_{1}+t_{2}+t_{3}\right) \\
& \left.+g_{e^{\prime} e}\left(t_{1}+t_{2}\right)+g_{e^{\prime} e}\left(t_{2}+t_{3}\right)-g_{e^{\prime} e}\left(t_{2}\right)\right\} \\
& S^{2 \mathrm{Q}_{2}}=-\sum_{e^{\prime} e} \sum_{f}\left\langle\mu_{e} \mu_{e f} \mu_{f e^{\prime}} \mu_{e^{\prime}}\right\rangle \\
& \times \exp \left\{-\mathrm{i} \omega_{e g} t_{1}-\mathrm{i} \omega_{f g} t_{2}+\mathrm{i} \omega_{e^{\prime} f} t_{3}\right. \\
& +\operatorname{conj}\left[-g_{e e}^{*}\left(t_{1}\right)-g_{f f}^{*}\left(t_{2}+t_{3}\right)-g_{e f}^{*}\left(t_{1}+t_{2}+t_{3}\right)\right. \\
& +g_{e f}^{*}\left(t_{1}\right)+g_{e f}^{*}\left(t_{2}+t_{3}\right)-g_{e e^{\prime}}^{*}\left(t_{1}+t_{2}\right)-g_{e^{\prime} e^{\prime}}\left(t_{3}\right) \\
& +g_{e e^{\prime}}^{*}\left(t_{1}+t_{2}+t_{3}\right)+g_{e e^{\prime}}^{*}\left(t_{2}\right)-g_{e e^{\prime}}^{*}\left(t_{2}+t_{3}\right) \\
& \left.\left.-g_{f e^{\prime}}^{*}\left(t_{2}\right)+g_{f e^{\prime}}^{*}\left(t_{2}+t_{3}\right)+g_{f e^{\prime}}\left(t_{3}\right)\right]\right\}
\end{aligned}
$$

$$
\begin{aligned}
& S^{\mathrm{SE}_{\mathrm{c}}}=\sum_{e^{\prime} \neq e}\left\langle\left|\mu_{e}\right|^{2}\left|\mu_{e^{\prime}}\right|^{2}\right\rangle \\
& \quad \times \exp \left\{-\mathrm{i} \omega_{e^{\prime} g} t_{1}+\mathrm{i} \omega_{e e^{\prime}} t_{2}-\mathrm{i} \omega_{e^{\prime} g} t_{3}\right. \\
& \quad-g_{e e}^{*}\left(t_{2}\right)-g_{e^{\prime} e^{\prime}}\left(t_{1}+t_{2}+t_{3}\right)-g_{e e^{\prime}}\left(t_{1}\right) \\
& \left.\quad+g_{e e^{\prime}}^{*}\left(t_{2}+t_{3}\right)+g_{e e^{\prime}}\left(t_{1}+t_{2}\right)-g_{e e^{\prime}}^{*}\left(t_{3}\right)\right\}
\end{aligned}
$$$$
S^{\mathrm{GSB}}=\sum_{e e^{\prime}}\left\langle\left|\mu_{e}\right|^{2}\left|\mu_{e^{\prime}}\right|^{2}\right\rangle
$$

$$
\begin{aligned}
& \times \exp \left\{-\mathrm{i} \omega_{e^{\prime} g} t_{1}-\mathrm{i} \omega_{e g} t_{3}\right. \\
& -g_{e e}\left(t_{3}\right)-g_{e^{\prime} e^{\prime}}\left(t_{1}\right)-g_{e e^{\prime}}\left(t_{1}+t_{2}+t_{3}\right) \\
& \left.+g_{e e^{\prime}}\left(t_{2}+t_{3}\right)+g_{e e^{\prime}}\left(t_{1}+t_{2}\right)-g_{e e^{\prime}}\left(t_{2}\right)\right\}
\end{aligned}
$$

\section{References}

[1] D.P. Weitekamp, K.Duppen, and D.A. Wiersma, Delayed four-wave-mixing spectroscopy in molecular crystals: A nonperturbative approach, Phys. Rev. A 27, 3089-3111 (1983).

[2] M. Cho, N.F. Scherer, G.R. Fleming, and S. Mukamel, Photon echoes and related four-wave-mixing spectroscopies using phase-locked pulses, J. Chem. Phys. 96, 5618-5629 (1992).

[3] M. Cho, H.M. Vaswani, T. Brixner, J. Stenger, and G.R. Fleming, Exciton analysis in 2d electronic spectroscopy, J. Phys. Chem. B 109, 10542-10556 (2005).

[4] D. Zigmantas, E.L. Read, T. Mančal, T. Brixner, A.T. Gardiner, R.J. Cogdell, and G.R. Fleming, Twodimensional electronic spectroscopy of the B800-B820 light-harvesting complex, Proc. Natl. Acad. Sci. USA 103, 12672-12677 (2006).

[5] V.I. Prokhorenko, A. Halpin, and R.J.D. Miller, Coherently-controlled two-dimensional photon echo electronic spectroscopy, Opt. Express 17, 9764-9779 (2009). 
[6] B.S. Prall, D.Y. Parkinson, G.R. Fleming, M. Yang, and N. Ishikawa, Two-dimensional optical spectroscopy: Two-color photon echoes of electronically coupled phthalocyanine dimers, J. Chem. Phys. 120, 2537-2540 (2004).

[7] M. Cho and G.R. Fleming, The integrated photon echo and solvation dynamics. II. Peak shifts and twodimensional photon echo of a coupled chromophore system, J. Chem. Phys. 123, 114506 (2005).

[8] P. Kiellberg, B. Brüggemann, and T. Pullerits, Twodimensional electronic spectroscopy of an excitonically coupled dimer, Phys. Rev. B 74, 024303 (2006).

[9] D.V. Voronine, D. Abramavicius, and S. Mukamel, Coherent control of cross-peaks in chirality-induced twodimensional optical signals of excitons, J. Chem. Phys. 125, 224504 (2006).

[10] A.V. Pisliakov, T. Mančal, and G.R. Fleming, Twodimensional optical three-pulse photon echo spectroscopy. II. signatures of coherent electronic motion and exciton population transfer in dimer twodimensional spectra, J. Chem. Phys. 124, 234505 (2006).

[11] Y.-C. Cheng and G.R. Fleming, Coherence quantum beats in two-dimensional electronic spectroscopy, J. Phys. Chem. A 112, 4254-4260 (2008).

[12] D. Abramavicius, V. Butkus, J. Bujokas, and L. Valkunas, Manipulation of two-dimensional spectra of excitonically coupled molecules by narrow-bandwidth laser pulses, Chem. Phys. 372, 22-32 (2010).

[13] T. Brixner, J. Stenger, H.M. Vaswani, M. Cho, R.E. Blankenship, and G.R. Fleming, Twodimensional spectroscopy of electronic couplings in photosynthesis, Nature 434, 625-628 (2005).

[14] G.S. Engel, T.R. Calhoun, E.L. Read, T.K. Ahn, T. Mančal, Y.C. Cheng, R.E. Blankenship, and G.R. Fleming, Evidence for wavelike energy transfer through quantum coherence in photosynthetic systems, Nature 446, 782 (2007).

[15] V.I. Prokhorenko, A.R. Holzwarth, F.R. Nowak, and T.J. Aartsma, Growing-in of optical coherence in the FMO antenna complexes, J. Phys. Chem. B 106, 99239933 (2002).

[16] E. Collini and G.D. Scholes, Coherent intrachain energy migration in a conjugated polymer at room temperature, Science 323, 369-373 (2009).

[17] J.M. Womick, S.A. Miller, and A.M. Moran, Probing the dynamics of intraband electronic coherences in cylindrical molecular aggregates, J. Phys. Chem. A 113, 6587-6598 (2009).

[18] F. Milota, J. Sperling, A. Nemeth, and H.F. Kauffmann, Two-dimensional electronic photon echoes of a double band J-aggregate: Quantum oscillatory motion versus exciton relaxation, Chem. Phys. 357, 45-53 (2009).

[19] I. Stiopkin, T. Brixner, M. Yang, and G.R. Fleming, Heterogeneous exciton dynamics revealed by two- dimensional optical spectroscopy, J. Phys. Chem. B 110, 20032-20037 (2006).

[20] P.F. Tian, D. Keusters, Y. Suzaki, and W.S. Warren, Femtosecond phase-coherent two-dimensional spectroscopy, Science 300, 1553-1555 (2003).

[21] T. Brixner, T. Mančal, I.V. Stiopkin, and G.R. Fleming, Phase-stabilized two-dimensional electronic spectroscopy, J. Chem. Phys. 121, 4221-4236 (2004).

[22] S. Mukamel, Multidimensional gemtosecond correlation spectroscopies of electronic and vibrational excitations, Annu. Rev. Phys. Chem. 51, 691-729 (2000).

[23] D.M. Jonas, Two-dimensional femtosecond spectroscopy, Annu. Rev. Phys. Chem. 54, 425-463 (2003).

[24] M. Cho, Coherent two-dimensional optical spectroscopy, Chem. Rev. 108, 1331-1418 (2008).

[25] B. Brüggemann, P. Kjellberg, and T. Pullerits, Nonperturbative calculation of 2d spectra in heterogeneous systems: Exciton relaxation in the fmo complex, Chem. Phys. Lett. 444, 192-196 (2007).

[26] D. Abramavicius, D.V. Voronine, and S. Mukamel, Unraveling coherent dynamics and energy dissipation in photosynthetic complexes by 2D spectroscopy, Biophys. J. 94, 3613-3619 (2008).

[27] D. Abramavicius, B. Palmieri, D.V. Voronine, F. Šanda, and S. Mukamel, Coherent multidimensional optical spectroscopy of excitons in molecular aggregates; Quasiparticle versus supermolecule perspectives, Chem. Rev. 109, 2350-2408 (2009).

[28] T. Brixner, I.V. Stiopkin, and G.R. Fleming, Tunable two-dimensional femtosecond spectroscopy, Opt. Lett. 29, 884-886 (2004).

[29] S. Mukamel, Principles of Nonlinear Optical Spectroscopy (Oxford University Press, New York, 1995).

[30] D. Kohen, C.C. Marston, and D.J. Tannor, Phase space approach to theories of quantum dissipation, J. Chem. Phys. 107, 5236-5253 (1997).

[31] M. Khalil, N. Demirdöven, and A. Tokmakoff, Coherent 2D IR spectroscopy: Molecular structure and dynamics in solution, J. Phys. Chem. A 107, 5258-5279 (2003).

[32] D. Abramavicius, L. Valkunas, and S. Mukamel, Transport and correlated fluctuations in the nonlinear optical response of excitons, Europhys. Lett. 80, 17005 (2007).

[33] M. Yang and G.R. Fleming, Influence of phonons on exciton transfer dynamics: comparison of the Redfield, Förster, and modified Redfield equations, Chem. Phys. 282, 163-180 (2002).

[34] H. van Amerongen, L. Valkunas, and R. van Grondelle, Photosynthetic Excitons (World Scientific, Singapore, 2000).

[35] A. Davydov, Theory of Molecular Excitons (McGrawHill, New York, 1962).

[36] R.E. Blankenship, Molecular Mechanisms of Photosynthesis (Blackwell Science, 2002).

[37] E.E. Jelley, Spectral absorption and fluorescence of dyes in the molecular state, Nature 138, 1009 (1936). 
[38] G. Scheibe, Über die Veränderlichkeit der Absorptionsspektren in Lösungen und die Nebenvalenzen als ihre Ursache, Angew. Chem. 49, 563 (1936).

[39] J-Aggregates, ed. T. Kobayashi (World Scientific, Singapore, 1996).

[40] E.W. Knapp, Lineshapes of molecular aggregates, exchange narrowing and intersite correlation, Chem. Phys. 85, 73-82 (1984).

[41] V.A. Malyshev and F. Domínguez-Adame, Motional narrowing effect in one-dimensional Frenkel chains with configurational disorder, Chem. Phys. Lett. 313, 255-260 (1999).

[42] H. Fidder, J. Knoester, and D.A. Wiersma, Optical properties of disordered molecular aggregates: A numerical study, J. Chem. Phys. 95, 7880-7890 (1991).

[43] H. Fidder, Absorption and emission studies on pure and mixed J-aggregates of pseudoisocyanine, Chem. Phys. 341, 158-168 (2007).

[44] R.E. Fenna and B.W. Matthews, Chlorophyll arrangement in a bacteriochlorophyll protein from Chlorobium limicola, Nature 258, 573-577 (1975).

[45] E.L. Read, G.S. Schlau-Cohen, G.S. Engel, J. Wen, R.E. Blankenship, and G.R. Fleming, Visualization of excitonic structure in the Fenna-Matthews-Olson pho- tosynthetic complex by polarization-dependent twodimensional electronic spectroscopy, Biophys. J. 95, 847-856 (2008).

[46] J. Olsina and T. Mančal, Electronic coherence dephasing in excitonic molecular complexes: Role of Markov and secular approximations, J. Mol. Model. (2010) [submitted].

[47] A. Ishizaki and G.R. Fleming, Theoretical examination of quantum coherence in a photosynthetic system at physiological temperature, Proc. Natl. Acad. Sci. USA 106, 17255-17260 (2009).

[48] A. Ishizaki and G.R. Fleming, Unified treatment of quantum coherent and incoherent hopping dynamics in electronic energy transfer: Reduced hierarchy equation approach, J. Chem. Phys. 130, 234111 (2009).

[49] A. Camara-Artigas, R.E. Blankenship, and J.P. Allen, The structure of the FMO protein from Chlorobium tepidum at $2.2 \AA$ resolution, Photosynth. Res. 75, 49 (2003).

[50] D. Abramavicius, B. Palmieri, and S. Mukamel, Extracting single and two-exciton couplings in photosynthetic complexes by coherent two-dimensional electronic spectra, Chem. Phys. 357, 79-84 (2009).

\title{
DVIMATE் OPTINĖ MOLEKULINIŲ AGREGATŲ SPEKTROSKOPIJA
}

\author{
V. Butkus a, D. Abramavičius ${ }^{\text {a,b }}$, A. Gelžinis a , L. Valkūnas a,c \\ ${ }^{a}$ Vilniaus universiteto Fizikos fakulteto Teorinès fizikos katedra, Vilnius, Lietuva \\ ${ }^{\mathrm{b}}$ Jilin universiteto Molekuliniu dariniu laboratorija, Čangčunas, Kinijos Liaudies Respublika \\ ${ }^{\mathrm{c}}$ Fizikos institutas, Fiziniu ir technologijos mokslu centras, Vilnius, Lietuva
}

\section{Santrauka}

Dvimatė elektroninė spektroskopija yra vienas naujausių bei pažangiausių daugiaimpulsinès spektroskopijos metodų, šiuo metu intensyviai naudojamas analizuojant vyksmus itin sudettinguose fotosintetiniuose dariniuose. Šis metodas leidžia geriau suprasti koherentiškumo gesimo ir užpildu pernašos vyksmus, stebimus sudètingose daugiachromoforinėse eksitoninio ryšio sistemose.

Pagrindiniai šio spektroskopijos metodo privalumai yra puiki laikinė skiriamoji geba (stebimi femtosekundžių trukmės vyksmai) bei koherentinès ir nekoherentinės prigimties sąveikaujančiu sistemų evoliucijų atskyrimas. Dèl pastarosios savybès galima stebèti sužadinimo pernašą sistemos viduje, itin efektyvų energijos perdavimą lemiančias koherentiškumo osciliacijas bei dar geriau išskirti nevienalytiškai išplitusias spektro linijas.

Iš (bakterio)chlorofilų sudarytuose bakterijų ir augalų fotosintezès reakciniuose centruose, šviesos energijos surinkimo bei perdavimo kompleksuose chromoforu skaičius yra didelis, tad iki šiol naudota supaprastinta netiesinio atsako teorija, išvystyta daug mažesnèms struktūroms, pavyzdžiui, dimui, aprašyti, nėra tiesiogiai taikytina. Tokių kompleksų modeliavimas tampa sudètingas, todèl taikomos ivairios aproksimacijos, naudojamas eksitoninis vaizdavimas.
Šiame darbe yra pristatoma bendra keturių bangų maišymo eksperimento modeliavimo schema bei išvestos trečios eilès atsako funkcijos išraiškos daugelio lygmenų eksitoninei sistemai, trumpai aptartos alternatyvios teorijos, paremtos neperturbaciniu kinetinès tankio matricos lygties sprendimu. Pateikiami sumodeliuoti elementarių kvantinių sistemų dvimačiai spektrai bei analizinès trečios eilès atsako išraiškos. Atskirai aptariami dimeru spektrai, kai juos sudarančios chromoforos aproksimuojamos kaip dviejų arba trijų lygmenų sistemos. Dviejų lygmenų chromoforų dimero atveju demonstruojamos galimybės pritaikyti dvispalvę dvimatę spektroskopiją siekiant išskirti spektrinius elementus bei indukuoti skirtingus fizikinius vyksmus sistemoje. Tokių matavimų rezultatai sudarytu prielaidas i̇vertinti makroskopinius nagrinejjamos sitemos parametrus - rezonansinę sąveiką, dipolinius momentus. Dvimatès spektroskopijos pritaikymui molekuliniams agregatams pateikiami vienmačio tiesinio J agregato bei fotosintetinio Fenna-MatthewsOlson (FMO) komplekso sugerties ir dvimačiai spektrai. Iš sumodeliuotų FMO komplekso spektrų galima daryti išvadas apie sužadinimo perdavimo tarp chromoforu scenarijus sistemoje bei koherentiškumo fliuktuacijas, kurios, manoma, lemia itin efektyvų energijos perdavimą sistemoje. 